\title{
Characterizing the continuous gravitational-wave signal from boson clouds around Galactic isolated black holes
}

\author{
Sylvia J. Zhu $\oplus^{1,2,3,{ }^{*}}$ Masha Baryakhtar®, ${ }^{4, \dagger}$ Maria Alessandra Papa $\oplus^{1,2,5, \$}$ Daichi Tsuna, ${ }^{6,7, \S}$ \\ Norita Kawanaka๑, ${ }^{8,9}$ and Heinz-Bernd Eggenstein $\oplus^{1,2}$ \\ ${ }^{1}$ Max-Planck-Institut für Gravitationsphysik, Callinstraße 38, D-30167 Hannover, Germany \\ ${ }^{2}$ Leibniz Universität Hannover, D-30167 Hannover, Germany \\ ${ }^{3}$ DESY, D-15738 Zeuthen, Germany \\ ${ }^{4}$ Center for Cosmology and Particle Physics, Department of Physics, New York University, \\ New York, New York 10003, USA \\ ${ }^{5}$ University of Wisconsin-Milwaukee, Milwaukee, Wisconsin 53201, USA \\ ${ }^{6}$ Research Center for the Early Universe (RESCEU), The University of Tokyo, \\ Hongo, Tokyo 113-0033, Japan \\ ${ }^{7}$ Department of Physics, School of Science, The University of Tokyo, Hongo, Tokyo 113-0033, Japan \\ ${ }^{8}$ Department of Astronomy, Graduate School of Science, Kyoto University, Kitashirakawa Oiwake-cho, \\ Sakyo-ku, Kyoto 606-8502, Japan \\ ${ }^{9}$ Hakubi Center, Yoshida Honmachi, Sakyo-ku, Kyoto 606-8501, Japan
}

(Received 26 March 2020; accepted 31 August 2020; published 21 September 2020)

\begin{abstract}
Ultralight bosons can form large clouds around stellar-mass black holes via the superradiance instability. Through processes such as annihilation, these bosons can source continuous gravitationalwave signals with frequencies within the range of LIGO and Virgo. If boson annihilation occurs, then the Galactic black hole population will give rise to many gravitational signals; we refer to this as the ensemble signal. We characterize the ensemble signal as observed by the gravitational-wave detectors; this is important because the ensemble signal carries the primary signature that a continuous wave signal has a boson annihilation origin. We explore how a broad set of black hole population parameters affects the resulting spin-0 boson annihilation signal and consider its detectability by recent searches for continuous gravitational waves. A population of $10^{8}$ black holes with masses up to $30 \mathrm{M}_{\odot}$ and a flat dimensionless initial spin distribution between zero and unity produces up to 1000 signals loud enough in principle to be detected by these searches. For a more moderately spinning population, the number of signals drops by about an order of magnitude, still yielding up to 100 detectable signals for some boson masses. A nondetection of annihilation signals at frequencies between 100 and $1200 \mathrm{~Hz}$ disfavors the existence of scalar bosons with rest energies between $2 \times 10^{-13}$ and $2.5 \times 10^{-12} \mathrm{eV}$. Finally, we show that, depending on the black hole population parameters, care must be taken in assuming that the continuous wave upper limits from searches for isolated signals are still valid for signals that are part of a dense ensemble: Between 200 and $300 \mathrm{~Hz}$, we urge caution when interpreting a null result for bosons between $4 \times 10^{-13}$ and $6 \times 10^{-13} \mathrm{eV}$.
\end{abstract}

DOI: $10.1103 /$ PhysRevD.102.063020

\section{INTRODUCTION}

*sylvia.zhu@desy.de

†mbaryakhtar@nyu.edu

*maria.alessandra.papa@aei.mpg.de

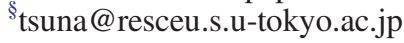

Published by the American Physical Society under the terms of the Creative Commons Attribution 4.0 International license. Further distribution of this work must maintain attribution to the author(s) and the published article's title, journal citation, and DOI. Open access publication funded by the Max Planck Society.
In the last few years, transient and rapidly evolving gravitational waves have been observed from the mergers of stellar-mass compact objects [1]. Persistent and slowly evolving sources of gravitational waves have been predicted as well and have yet to be detected. These continuous gravitational waves are expected to be much weaker than the transient events and have durations much longer than the typical observation time; searches for continuous waves integrate over long periods of time to extract signal from background. 
Canonical sources of continuous waves include neutron stars with mass deformations or internal fluid oscillation modes (see [2] for a recent review). Scenarios involving more exotic emitters of continuous waves are also being explored and can provide evidence for-or disfavor the presence of - new physics beyond the Standard Model of particle physics [3-7]. A particularly well-motivated target is the axion, proposed to solve the strong- $C P$ problem in particle physics [8-10]; axions and axionlike particles are also promising dark matter candidates (see, e.g., [11] for a review).

Bosons such as axions or axionlike particles can form "clouds" with enormous occupation numbers around rotating black holes, and they do so rapidly on astrophysical timescales when the black hole size is similar to the boson's Compton wavelength $[3,4]$. These boson-black hole systems can be thought of as gravitational "atoms," and within this scenario, boson annihilations and level transitions source monochromatic gravitational-wave emission $[3,4]$. Annihilations in particular produce signals that may be loud enough to be detected in standard continuous wave searches on data from the current generation of groundbased interferometers [12-15]. Bosons with masses of $\sim 10^{-13}$ to $4 \times 10^{-12} \mathrm{eV}$ produce annihilation signals with frequencies between $\sim 50$ and $2000 \mathrm{~Hz}$, which is in the LIGO and Virgo band.

The boson cloud forms by extracting energy and angular momentum from the black hole, which loses a significant fraction of its natal spin $[3,4]$. Measurements of the spins of several old, highly rotating black holes in X-ray binaries $[16,17]$ have been used to disfavor the range of masses $6 \times 10^{-13} \mathrm{eV} \lesssim \mu_{\mathrm{b}} \lesssim 2 \times 10^{-11} \mathrm{eV}[12,18]$. However, since the spin measurements come with a set of systematic uncertainties, it is very important to undertake complementary searches. Direct continuous wave searches for the annihilation signal in LIGO and Virgo data are an independent test in the boson mass range disfavored by rapidly rotating black holes, and they may be able to extend the reach to lower masses, as well as discover a new particle.

In order to disfavor a range of boson masses or characterize a potential continuous wave signal, it is crucial to consider the ensemble signal from the population of sources as a whole. The properties of the annihilation signal depend strongly on the mass, spin, distance, and age of each black hole, so the properties of the ensemble signal depend on the properties of the black hole population. Moreover, to leading order, the annihilation signal frequency is set by the boson rest mass, so the emission from all boson clouds falls within a small frequency range. This is in contrast to continuous waves from neutron stars, which are expected to span a broad range of frequencies depending on the rotation rates of the individual stars. The clustering of signals in a narrow frequency band could reduce the effectiveness of continuous wave search methods-which are optimized for weak, isolated signals-in identifying the annihilations, and it may recommend the use of other methods entirely (see, e.g., $[15,19]$ ).

In this paper, we study the expected boson annihilation signal from the population of isolated black holes in the Galaxy, of which there are expected to be up to $\sim 10^{8}$. In order to take all effects into account, we use simulated populations of $10^{8}$ black holes and calculate the expected signal from all of the systems for bosons with energies between $1 \times 10^{-13}$ and $4 \times 10^{-12} \mathrm{eV}$, corresponding approximately to gravitational-wave frequencies between 50 and $2000 \mathrm{~Hz}$. We investigate the detectability of the ensemble signal in current LIGO data by broad continuous gravitational-wave surveys [20-23], and its dependence on black hole population assumptions.

We review the theory of boson cloud formation and continuous wave emission in Sec. II and present the Galactic isolated black hole population in Sec. III. We explore the resultant ensemble signal from the entire population in Sec. IV, study its detectability in Sec. V, compare our results to the current literature in Sec. VI, and summarize in Sec. VII.

\section{SIGNAL MODEL}

"Gravitational atoms"-macroscopic, gravitationally bound states of ultralight bosons around astrophysical black holes-form rapidly via the superradiance instability. The bosons subsequently annihilate, sourcing coherent, monochromatic, and long-lasting gravitational waves $[3,4,12]$. The frequency of the signal at leading order is given by twice the boson's rest energy,

$$
f_{\mathrm{GW}}^{0}=\frac{2 \mu_{\mathrm{b}}}{h} \approx 48.3 \mathrm{~Hz}\left(\frac{\mu_{\mathrm{b}}}{10^{-13} \mathrm{eV}}\right),
$$

with $\mu_{\mathrm{b}}=m_{\mathrm{b}} c^{2}, m_{\mathrm{b}}$ the boson mass, $c$ the speed of light, and $h$ the Planck constant. In this work, we focus on signals from gravitationally interacting scalar (spin-0) bosons around stellar-mass black holes; we use "black hole" to exclusively refer to stellar-mass black holes throughout the text. The growth and annihilation timescales of vector (spin-1) [24-28] and spin-2 [29] bosons are shorter and require a separate analysis. We consider annihilation signals only from the fastest-growing bound state and limit our analysis to isolated black holes without external effects such as accretion or binary companions.

We summarize the necessary background below and provide further details in the Appendix A; see, e.g., $[4,12]$ for more details and [30] for a review.

\section{A. Cloud formation}

Black hole superradiance is a purely kinematic process [31] whereby a wave scattering off a rapidly rotating black hole increases in amplitude by extracting some of the black hole's angular momentum [32,33]. The gravitational 


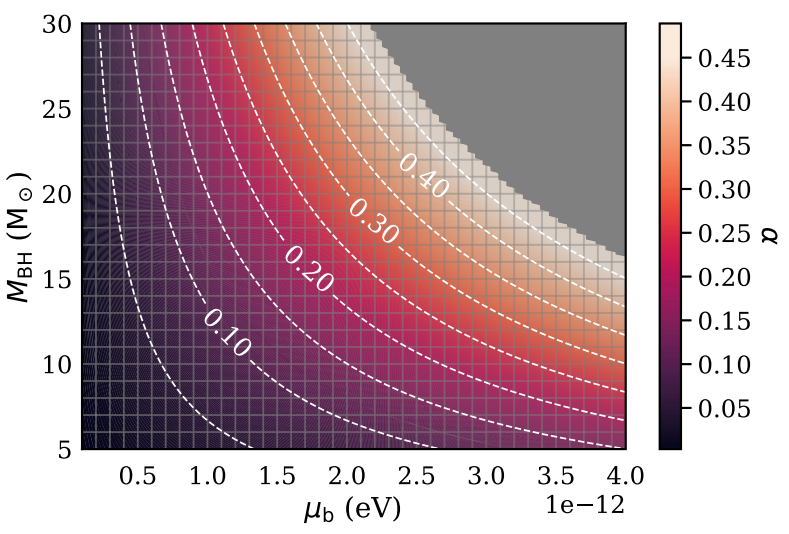

FIG. 1. Gravitational fine structure constant $\alpha \equiv G M_{\mathrm{BH}} \mu_{\mathrm{b}} /(\hbar c)$. Given the superradiance condition, the $(n, \ell, m)=(0,1,1)$ bound state cannot form for $\alpha \geq 0.5$, which is shown in gray.

potential of the black hole enables massive particle bound states, and the amplitudes of bound states that satisfy the "superradiance condition" increase [34-37]. For bosons, the growth is exponential and results in a cloud with a macroscopic number of particles all occupying the same state. The initial seed can be a vacuum fluctuation, so the process need not rely on an existing abundance of bosons in the black hole's vicinity.

The bound states are approximated by hydrogenic wave functions, characterized by radial, orbital, and azimuthal quantum numbers $(n, \ell, m)$ and the gravitational analog of the "fine structure constant," $\alpha$,

$$
\alpha \equiv \frac{G M_{\mathrm{BH}} \mu_{\mathrm{b}}}{\hbar c^{3}} \approx 0.0075\left(\frac{M_{\mathrm{BH}}}{10 \mathrm{M}_{\odot}}\right)\left(\frac{\mu_{\mathrm{b}}}{10^{-13} \mathrm{eV}}\right)
$$

with $G$ the gravitational constant, $\hbar=h / 2 \pi, \mathrm{M}_{\odot}$ the mass of the Sun, and $M_{\mathrm{BH}}$ the mass of the black hole (Fig. 1).

Unless otherwise specified, we consider only the first superradiant level, with $(n, \ell, m)=(0,1,1) .{ }^{1}$ The level will grow if the initial spin of the black hole, $\chi_{i}$, satisfies the superradiance condition,

$$
\alpha \lesssim \frac{1}{2} \frac{\chi_{i}}{1+\sqrt{1-\chi_{i}^{2}}}
$$

The inequality is equivalent to the angular velocity of the black hole exceeding the angular velocity of the cloud. For a given $\alpha$, this is true when the initial spin of the black hole $\chi_{i}$ is greater than the critical spin $\chi_{c}$ (Fig. 2),

$$
\chi_{c} \approx \frac{4 \alpha}{1+4 \alpha^{2}} \text {. }
$$

\footnotetext{
${ }^{1}$ Some of the literature uses the principal number $\bar{n}=n+\ell+1$, with $(\bar{n}, \ell, m)=(2,1,1)$ the fastest-growing state.
}

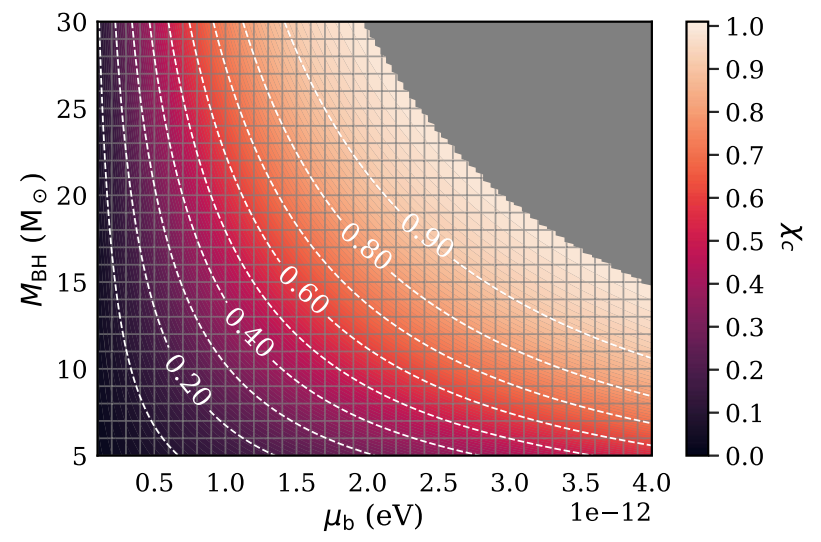

FIG. 2. Critical spin $\chi_{c}$ for the $(n, \ell, m)=(0,1,1)$ bound state. The critical spin is a function of the gravitational fine structure constant $\alpha$ and the bound state quantum numbers. A cloud of bosons with rest energy $\mu_{\mathrm{b}}$ will form around a black hole of mass $M_{\mathrm{BH}}$ and initial spin $\chi_{i}$ only if $\chi_{i}>\chi_{c}$ [Eq. (4)].

[See Eq. (A8) for gravitational potential energy corrections and dependence on bound state quantum numbers.] Thus, the first level will form for $\alpha \lesssim 0.5$, smaller for lower initial spins. However, only the range $0.03 \lesssim \alpha \lesssim 0.2$ contributes to signals that are simultaneously loud enough (Fig. 3) and sufficiently long lasting (Fig. 4) to be potentially detectable. For $\alpha \in[0.03,0.2], \chi_{c} \in[0.1,0.7]$.

The $(n, \ell, m)=(0,1,1)$ level has the largest annihilation power and the fastest growth time, with $e$-folding time for the number of particles $\tau_{\text {inst }}$ given by

$$
\tau_{\text {inst }} \approx 14 \text { days }\left(\frac{M_{\mathrm{BH}}}{10 \mathrm{M}_{\odot}}\right)\left(\frac{0.1}{\alpha}\right)^{9} \frac{1}{\chi_{i}}
$$

at leading order in $\alpha$; see also Eq. (A7). The instability timescale is very sensitive to the boson and black hole

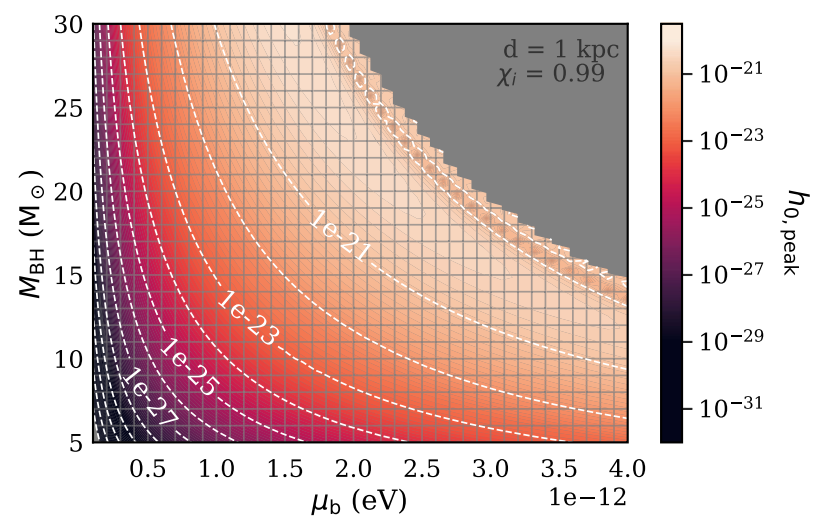

FIG. 3. Maximum characteristic strain $h_{0 \text {,peak }}$ for a rapidly rotating black hole as a function of black hole mass $M_{\mathrm{BH}}$ and boson mass $\mu_{\mathrm{b}}$ at a distance of $d=1 \mathrm{kpc}$. The peak strain scales as $1 / d$. The value of $h_{0}$ increases with $\alpha$ until $\alpha \sim 0.35$; the quadrupolar gravitational-wave power-and thus the observed strain-changes nonmonotonically at large $\alpha$ (Sec. A 4). 


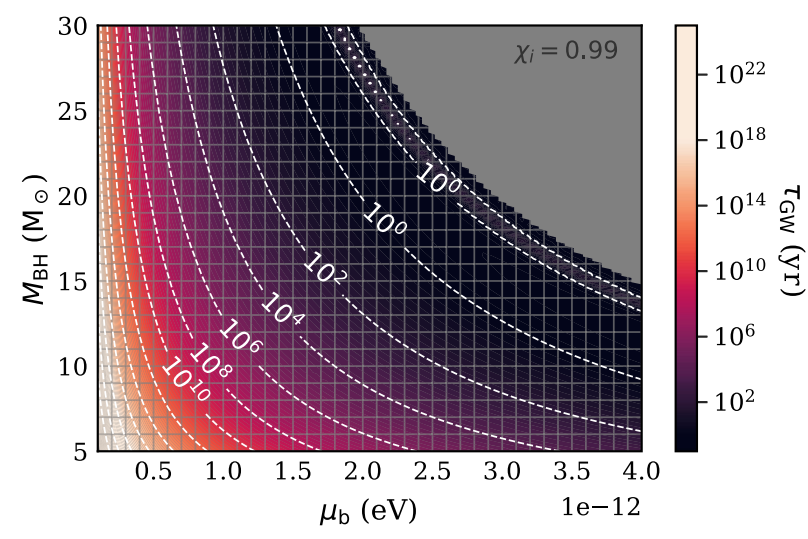

FIG. 4. The gravitational-wave signal amplitude decay halftime $\tau_{\mathrm{GW}}$ [Eq. (14)] ranges from longer than the Hubble time for lighter bosons to less than $100 \mathrm{yr}$ for heavier bosons. The longer signals from lighter bosons are weaker compared to shorter signals from heavier bosons [Eq. (13)]. Like $h_{0}, \tau_{\mathrm{gw}}$ is a nonmonotonic function of $\alpha$ above $\alpha \gtrsim 0.35$ (Sec. A 4).

masses, and systems with lighter bosons and lighter black holes take longer to form.

The cloud extracts angular momentum from the black hole until $\chi \simeq \chi_{c}$, at which point the cloud ceases to grow. The black hole loses mass as well as spin in this process, so the values of $\alpha$ and $\chi_{c}$ decrease slightly as the cloud grows; we take this evolution into account as described in Appendix A. At this point, the masses of the black hole and of the cloud are approximately

$$
\begin{gathered}
M_{\mathrm{BH}, f} \approx M_{\mathrm{BH}}\left(1-\alpha\left(\chi_{i}-\chi_{c}\right)\right), \\
M_{\text {cloud }} \approx M_{\mathrm{BH}} \alpha\left(\chi_{i}-\chi_{c}\right),
\end{gathered}
$$

where $M_{\mathrm{BH}}$ is the black hole mass at the onset of the cloud formation. For the systems of interest, $M_{\text {cloud }} \approx$ $(0.1 \%-5 \%) M_{\mathrm{BH}}$, which corresponds to

$$
N \simeq \frac{M_{\text {cloud }}}{\mu_{\mathrm{b}} / c^{2}} \approx 10^{77}\left(M_{\mathrm{BH}} / 10 \mathrm{M}_{\odot}\right)^{2}
$$

particles in the cloud. The timescale to fully populate the level is then $\ln (N) \tau_{\text {inst }} \sim 180 \tau_{\text {inst }}$.

\section{B. Gravitational-wave emission}

We study gravitational-wave signals emitted from the resultant bound state: Two bosons within the cloud can annihilate into a single graviton in the presence of the black hole gravitational field. "Transition" signals can also occur if multiple states are simultaneously populated, but they are less promising at current sensitivities [12]. We assume that the bosons interact only via gravity. ${ }^{2}$ The presence of

\footnotetext{
${ }^{2}$ For example, the QCD axion in this mass range has selfinteraction scale $\gtrsim 10^{18} \mathrm{GeV}$, so this assumption is valid.
}

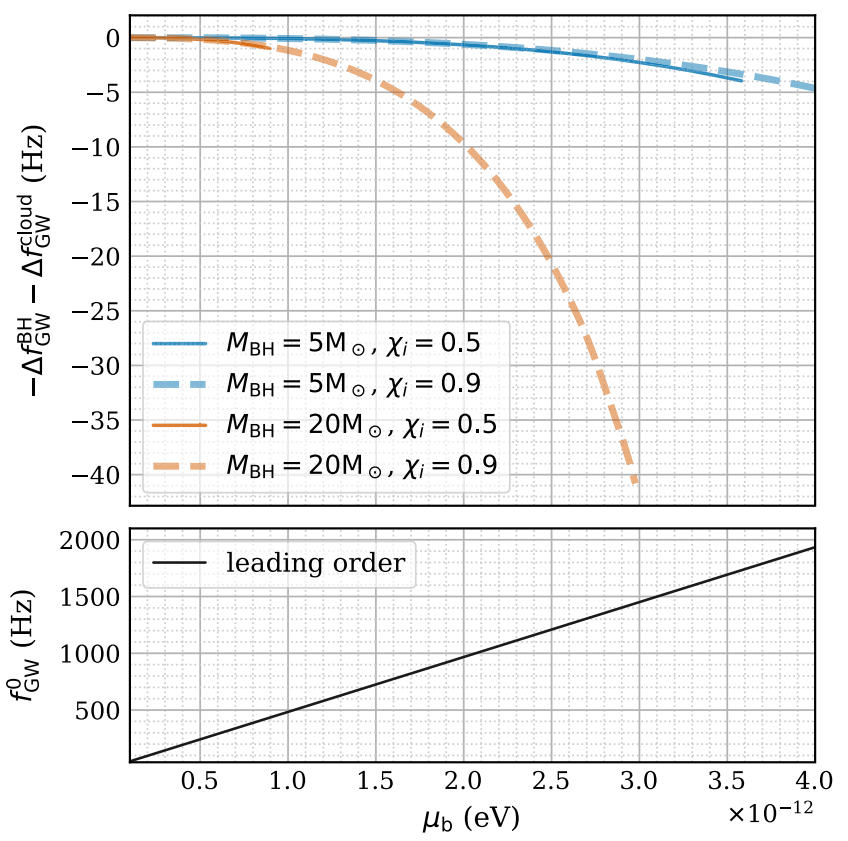

FIG. 5. Bottom panel: The leading-order gravitational-wave frequency $f_{\mathrm{GW}}^{0}$ is proportional to the boson rest energy $\mu_{\mathrm{b}}$ [Eq. (9)]. Top panel: Systems with heavier black holes (orange curves) produce GW signals with lower frequencies than do systems with lighter black holes (blue curves) due to the larger gravitational binding energy. Systems with larger spins (dashed curves) result in slightly higher frequencies than systems with smaller spins (solid curves) due to a positive spin-orbit energy. The two solid curves-corresponding to $\chi_{i}=0.5$ - stop at intermediate boson masses; for heavier bosons, $\chi_{c}>0.5$ and the systems never form. The contribution from the cloud selfenergy is of the order of $10^{-3}$ or less of the black hole's gravitational potential energy.

self-interactions can change the evolution and limit the size of the cloud [38,39], or potentially cause the collapse of the cloud in a "bosenova" $[4,40,41]$. The signal from the bosons in a single macroscopic bound state is coherent and monochromatic and evolves slowly, thus providing an ideal target for continuous wave searches. For lighter bosons and black holes, the signal properties are essentially unchanged over a Hubble time.

The frequency $f_{\mathrm{GW}}$ of the emitted gravitational wave is given by

$$
f_{\mathrm{GW}}=f_{\mathrm{GW}}^{0}-\Delta f_{\mathrm{GW}}^{\mathrm{BH}}-\Delta f_{\mathrm{GW}}^{\mathrm{cloud}},
$$

where the corrections to the leading-order frequency, Eq. (1), are due to the gravitational potential of the black hole [42,43] (see Fig. 5) and the cloud itself,

$$
\begin{gathered}
\Delta f_{\mathrm{GW}}^{\mathrm{BH}} \approx f_{\mathrm{GW}}^{0}\left(\frac{\alpha^{2}}{8}+\frac{17 \alpha^{4}}{128}-\frac{\chi_{i} \alpha^{5}}{12}\right), \\
\Delta f_{\mathrm{GW}}^{\text {cloud }} \approx f_{\mathrm{GW}}^{0}\left(0.2 \alpha^{2} \frac{M_{\text {cloud }}}{M_{\mathrm{BH}}}\right) .
\end{gathered}
$$


The power emitted in gravitational waves for $\alpha \ll 1$ is given by [44]

$$
P_{\mathrm{GW}} \approx 0.025 \frac{c^{5}}{G} \alpha^{14} \frac{M_{\mathrm{cloud}}^{2}}{M_{\mathrm{BH}}^{2}} .
$$

The corrections at larger $\alpha$ are significant, and the power in this regime has been computed numerically $[14,45]$ [see Eq. (A16)]. The gravitational waves are produced approximately in a background defined by the final black hole mass, and we use the final value of $\alpha$ to evaluate the power, strain, and timescale expressions.

The characteristic strain of the annihilation signal is largest when the cloud first reaches its maximum occupation number. At leading order,

$$
h_{0, \text { peak }} \approx 3 \times 10^{-24}\left(\frac{\alpha}{0.1}\right)^{7}\left(\frac{\chi_{i}-\chi_{c}}{0.5}\right)\left(\frac{M_{\mathrm{BH}}}{10 \mathrm{M}_{\odot}}\right)\left(\frac{1 \mathrm{kpc}}{d}\right) \text {, }
$$

where $h_{0}$ is the maximal intrinsic gravitational-wave amplitude [Eqs. (A17) and (A18)]. The strain $h_{0, \text { peak }}$ thus rapidly increases for larger values of $\mu_{\mathrm{b}}, M_{\mathrm{BH}}$ (Fig. 3). For reference, recent all-sky continuous gravitational-wave searches have reported $h_{0}$ upper limits of $\approx\left\{2 \times 10^{-25}, 3 \times\right.$ $\left.10^{-25}, 6 \times 10^{-25}, 1 \times 10^{-24}\right\}$ at frequencies of $\{180,600$, $1000,2000\} \mathrm{Hz}$, respectively [20,21,46,47].

As the bosons annihilate and the cloud is depleted, the signal strength $h_{0}(t)$ decreases from its peak as [4]

$$
h_{0}(t)=\frac{h_{0, \text { peak }}}{1+t / \tau_{\mathrm{GW}}},
$$

where $\tau_{\mathrm{GW}}=M_{\text {cloud }}^{\text {peak }} c^{2} / P_{\mathrm{GW}}^{\text {peak }}$ is the signal "half-time,"

$$
\tau_{\mathrm{GW}} \approx 5 \times 10^{5} \operatorname{yr}\left(\frac{M_{\mathrm{BH}}}{10 \mathrm{M}_{\odot}}\right)\left(\frac{0.1}{\alpha}\right)^{15}\left(\frac{0.5}{\chi_{i}-\chi_{c}}\right) .
$$

The self-binding energy of the cloud decreases with decreasing cloud mass [Eq. (10)], giving a small positive first frequency derivative [also see Eq. (A20)],

$$
\dot{f}_{\mathrm{gw}}(t) \approx 0.2 \alpha \frac{f_{\mathrm{GW}}}{\tau_{\mathrm{GW}}}\left(\frac{M_{\text {cloud }}(t)}{M_{\mathrm{BH}}}\right)^{2} .
$$

In the relevant part of parameter space, the frequency drift is $\dot{f}_{\mathrm{gw}} \lesssim 10^{-12} \mathrm{~Hz} / \mathrm{s}$ (Fig. 23), which is smaller than what current all-sky continuous wave searches can resolve in their initial stages $[46,47]$. The frequency drift is discussed further in Sec. IVA.

\section{Second fastest growing bound state}

The second fastest growing bound state with $(n=0$, $\ell=m=2$ ) has parametrically longer growth and annihilation timescales (Appendix A). It also corresponds to a lower critical spin and thus will reduce the black hole spin below the first level's critical value $\chi_{c}$. The black hole then begins absorbing the first level bosons $[4,48]$, and the "spin-up" rate due to first level absorption balances the spin-down rate caused by the growth of the second level. Ultimately, enough of the first level is absorbed by the black hole that the annihilations effectively cease, and continuous wave emission shuts off [4]. We take this effect into account by setting $h_{0}(t)=0$ when the second level fully populates, $\sim \ln (N) \tau_{\text {inst }}^{022}$ after the black hole formation, where

$$
\tau_{\text {inst }}^{022} \approx 2 \times 10^{5} \operatorname{yr}\left(\frac{M_{\mathrm{BH}}}{10 \mathrm{M}_{\odot}}\right)\left(\frac{0.1}{\alpha}\right)^{13} \frac{1}{\chi_{i}\left(1+3 \chi_{i}^{2}\right)} .
$$

The cutoff time in the signal becomes important at $\alpha \gtrsim 0.1$, reducing the number of signals.

The second level also produces continuous waves, and given the lower critical spin, clouds can form in systems with smaller $\chi_{i}$ or larger boson masses. However, the strain is significantly weaker than for the first level; as described in Appendix A, $h_{0 \text {,peak }}^{011} / h_{0 \text {,peak }}^{022} \sim 90 / \alpha^{2}$ [45]. In addition, the emission from the $(n=0, \ell=m=2)$ level is no longer dominantly quadrupolar [45], which would require an ad hoc continuous wave search. For these reasons, we do not consider the gravitational-wave emission from the second fastest growing state in our study, although it would be interesting to do so in the future.

\section{THE BLACK HOLE POPULATION}

For a given boson mass, the annihilation signal properties depend on properties of the black hole: the distance $d$, velocity $v$, initial $M_{\mathrm{BH}}$, and initial spin $\chi_{i}$ [Eqs. (9) and (13)], and age $\tau_{\mathrm{BH}}$. The total number of black holes in the Galaxy is estimated to be around $10^{8}$ (see, e.g., [49,50]); based on black hole formation rates of $\sim 0.1-0.9$ per Milky Way type galaxy per century, the total number is typically estimated to vary between $10^{7}$ and $10^{8}$ [51-55], with some estimates being as high as $10^{9}$ [56]. The number of isolated black holes is unknown but is expected to be the same order of magnitude as the total number of black holes (see, e.g., [55-57]) We therefore take $10^{8}$ as the benchmark number of isolated black holes in the Galaxy, consistent with the latest simulations of Galactic black holes [55]; as the total number is uncertain, our results can be rescaled to draw conclusions on populations with a different number of black holes. To manage computation time, we simulate the positions and velocities of $10^{6}$ black holes and perform a "resampling" to scale up to $10^{8}$ isolated black holes in the Galaxy (Sec. III A).

\section{A. Simulating positions and velocities}

The spatial and velocity distribution of the current population of isolated Galactic black holes is not known. 
However, since the black holes formed from massive stars at an earlier time, we approximate the black holes' spatial distribution at birth with the current stellar distribution and evolve their trajectories, including initial bulk velocities and natal kicks, through the Galactic gravitational potential using the procedure described by Tsuna et al. [58]. The Galactic gravitational potential is divided into the bulge, disk, and halo components; the black holes are born in the bulge and disk according to their respective birth rates in the regions, and their positions and velocities are evolved through the Galactic gravitational potential. (See [58] and references therein for more details.) Direct measurement of isolated Galactic black holes, such as by radio observations $[58,59]$, would significantly reduce the uncertainties in their properties.

The disk is defined by a cylindrical coordinate system with radial coordinate $r$, height $z$, and azimuth $\phi$, while the halo is defined by a spherical coordinate system with radial coordinate $R$. The bulge is described by both cylindrical and spherical coordinate systems. All coordinates are defined with the Galactic Center at the origin. The black hole birth rate per unit volume in the bulge (subscript $b$ ) and disk (subscript $d$ ) are given the following prescriptions $[60,61]$ :

$$
\begin{gathered}
\dot{\rho}_{b}(R) \propto \exp \left(-R / R_{b}\right), \\
\dot{\rho}_{d}(r) \propto \exp \left(-r / r_{d}\right),
\end{gathered}
$$

where $R_{b}=120 \mathrm{pc}$ and $r_{d}=2.15 \mathrm{kpc} ; \dot{\rho}_{d}$ is uniform along $z$ for $|z|<75 \mathrm{pc}$. The proportionality factors in Eqs. (18) and (19) are determined by requiring that the total number of black holes born be $10^{6}$, with $15 \%$ born in the bulge and $85 \%$ in the disk [60]. $\dot{\rho}_{d}$ is taken to be constant over time, while $\dot{\rho}_{b}$ is nonzero and constant between 10 and 8 Gyr ago [62].

When a black hole is first born, it is given an initial velocity composed of a bulk velocity as well as an individual kick. The bulk velocity in the disk is a piecewise function of distance from the Galactic Center, in the $\phi$ direction [63],

$v_{\phi}\left(\mathrm{km} \mathrm{s}^{-1}\right)= \begin{cases}265-1875(r-0.2)^{2}, & r<0.2, \\ 225+15.625(r-1.8)^{2}, & 0.2<r<1.8, \\ 225+3.75(r-1.8), & 1.8<r<5.8, \\ 240, & r>5.8,\end{cases}$

where $r$ is in kiloparsecs. The bulk velocity in the bulge is taken from a Maxwell-Boltzmann distribution with a mean of $130 \mathrm{~km} / \mathrm{s}$ [64].

Asymmetries in the supernova explosion can impart a "kick" to the newly formed compact object. The natal kicks of Galactic neutron stars are known to be as large as hundreds of km/s [65]. Since black holes are many times heavier than neutron stars, relatively smaller natal kicks are expected, depending on the kick mechanism [66,67]. Recent studies of known Galactic stellar-mass black holes find that their properties are consistent with much smaller natal kicks than the ones attributed to neutron stars $[68,69]$ (although Repetto et al. [70] found similar natal kick distributions for black holes and neutron stars).

The black holes' initial kicks in our simulation follow a Maxwell-Boltzmann distribution with average 3D velocity of $50 \mathrm{~km} / \mathrm{s}$, the smallest average kick velocity used in [58]. This means that black holes tend to stay in the Galactic component in which they were born, and few black holes migrate to high Galactic latitudes. We also consider the impact of larger kick velocities in Appendix B, finding that natal kicks of $100 \mathrm{~km} / \mathrm{s}$ give slightly reduced signal numbers due to black holes with larger kicks having larger distance $d$ from the solar system, on average; $\langle 1 / d\rangle \sim$ $(7.8 \mathrm{kpc})^{-1}$ for the $50 \mathrm{~km} / \mathrm{s}$ population and $\langle 1 / d\rangle \sim$ $(8.3 \mathrm{kpc})^{-1}$ for the $100 \mathrm{~km} / \mathrm{s}$ population, corresponding to the signals from the latter population being about $10 \%$ weaker on average. See Appendix B for further discussion.

After a black hole is born in the simulation, its trajectory is determined by the Galactic gravitational potential. The gravitational potential of the bulge, disk, and halo (subscript $h$ ) are defined as follows [63]:

$$
\begin{aligned}
& \phi_{b}(r, z)=-\frac{G M_{b}}{\sqrt{r^{2}+\left(a_{b}+\sqrt{z^{2}+b_{b}^{2}}\right)^{2}}}, \\
& \phi_{d}(r, z)=-\frac{G M_{d}}{\sqrt{r^{2}+\left(a_{d}+\sqrt{z^{2}+b_{d}^{2}}\right)^{2}}}, \\
& \phi_{h}(R)=-\frac{G M_{h}}{R_{h}} \ln \left(\frac{\sqrt{R^{2}+R_{h}^{2}}+R_{h}}{R}\right),
\end{aligned}
$$

where $R \equiv \sqrt{r^{2}+z^{2}}$ and the constants are

$$
\begin{aligned}
& M_{b}=4.07 \times 10^{9} \mathrm{M}_{\odot}, \quad a_{b}=0 \mathrm{kpc}, \quad b_{b}=0.184 \mathrm{kpc}, \\
& M_{d}=6.58 \times 10^{10} \mathrm{M}_{\odot}, \quad a_{d}=4.85 \mathrm{kpc}, \quad b_{d}=0.305 \mathrm{kpc}, \\
& M_{h}=1.62 \times 10^{12} \mathrm{M}_{\odot}, \quad R_{h}=200 \mathrm{kpc},
\end{aligned}
$$

as determined by fits to observed Galactic properties (e.g., rotation curves) in [63].

With this procedure, we obtain the final positions and velocities of $10^{6}$ black holes with respect to the Galactic Center, but we require a full population of $10^{8}$ black holes with positions and velocities as measured from the Solar System barycenter (SSB). Since our simulations assume axial symmetry (i.e., we do not include any structure like 


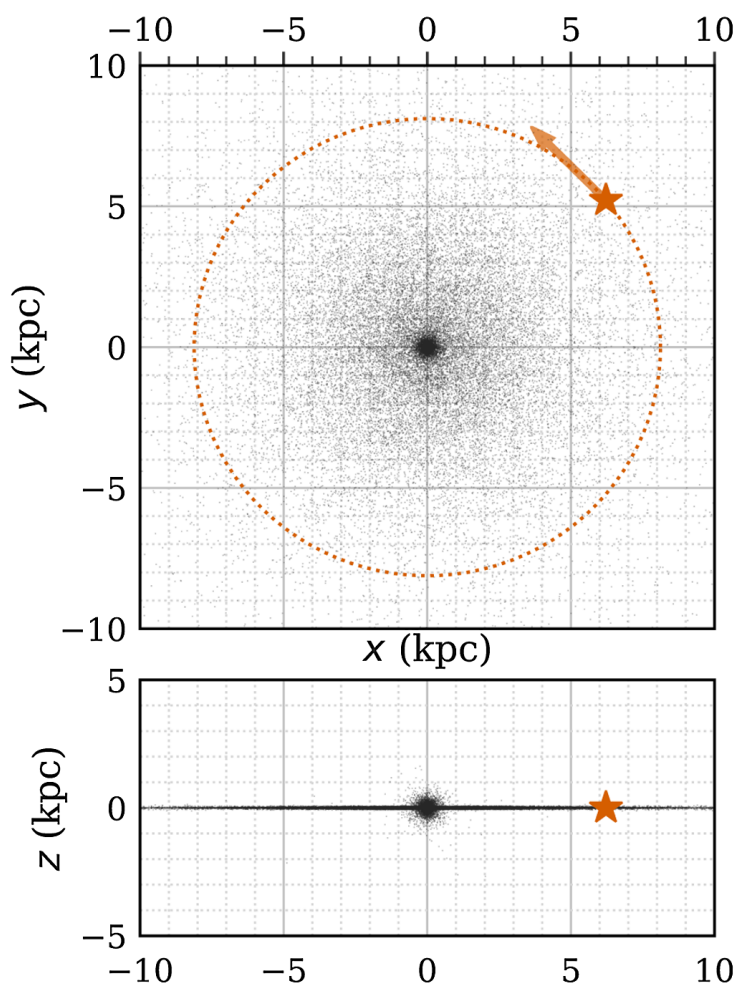

FIG. 6. Top-down (top panel) and edge-on (bottom panel) view of the Galaxy, showing a random sample of 30,000 black holes from our simulated population. The disk and bulge components are visually distinct. Our simulations assume axial symmetry, so the results do not change if the position of the Sun is chosen arbitrarily on the circle of distance $\sim 8 \mathrm{kpc}$ from the Galactic Center (orange circle). As explained in the text, we exploit this freedom to simulate $10^{8}$ black holes starting from a set of $10^{6}$. The orange star marks one sample position and the arrow shows its velocity in the disk, tangential to the circle.

the spiral arms), we are free to choose the position of the SSB to be anywhere $8.12 \mathrm{kpc}$ away from the center in the disk, with a velocity prescribed by Eq. (20). We therefore randomly select 100 points from the circle of radius $8.12 \mathrm{kpc}$, as illustrated in Fig. 6, and build up a sample of $10^{8}$ black holes at minimal computational cost increase. For the resulting black holes, we compute the distances $d$ and radial and tangential velocities $v_{\text {rad }}$ and $v_{\text {tan }}$ as measured from the SSB.

We require that the individual black holes be independent; to check that the distribution is not oversampled, we estimate that, on average, the distance between one SSB position assignment and the next is $500 \mathrm{pc}$. In comparison, the average separation between black holes is $\mathcal{O}(10 \mathrm{pc})$, assuming that the $10^{8}$ black holes are uniformly distributed in the Galactic plane. The average separation between black holes is therefore over an order of magnitude smaller than the average separation between SSB position assignments, so the local black hole populations that are "seen" at given SSB position assignments are largely independent of one another.

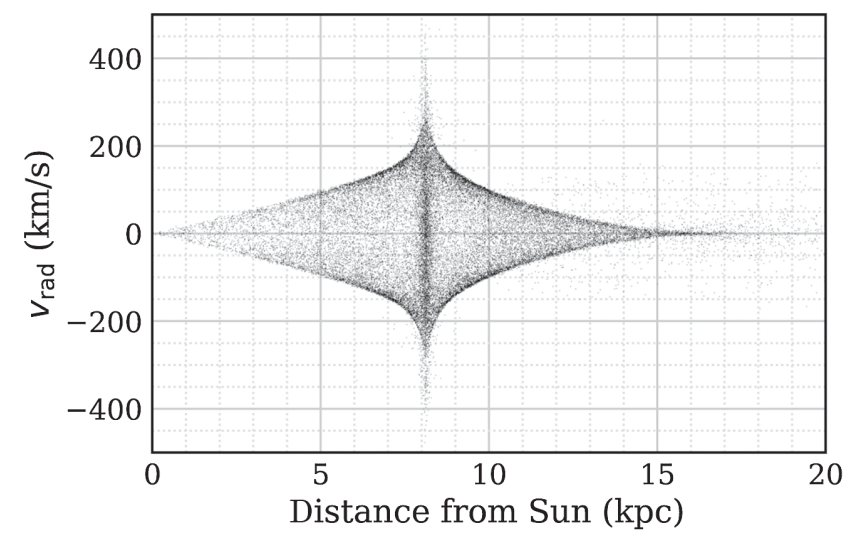

FIG. 7. Radial velocities of the black holes of Fig. 6 as a function of their distance from the Sun. The distribution is symmetric around zero $\mathrm{km} / \mathrm{s}$ and is widest at $8 \mathrm{kpc}$, corresponding to the distance of the Galactic Center, as the bulk velocity in the bulge is uniformly distributed in direction. In contrast, since the bulk velocity in the disk is in the $\phi$ direction within the Galactic plane, the radial velocity of black holes in the disk (i.e., not near $8 \mathrm{kpc}$ ) has smaller magnitude. The scatter in velocities at large distances from the Sun is due to the black holes that have wandered into the halo and no longer follow the bulk motion of the disk.

While this procedure effectively reproduces the spatial and velocity distribution of $10^{8}$ black holes using only $10^{6}$ simulated objects, it does not accurately reproduce the age distribution of young black holes. Given the assumption of a constant formation rate in the last $8 \mathrm{Gyrs}$, a population of $10^{6}$ black holes underestimates the number with age $10^{4}$ years or less compared to that which would be produced by a full simulation of $10^{8}$ objects. This effect is relevant for heavier bosons, which have short radiation timescales compared to the typical age of a black hole, and is discussed further in Sec. IV C.

\section{B. Choice of mass distribution}

For the mass distribution, we use the Salpeter function, $d N / d M \propto M^{-2.35}$ [71], an empirically determined function that has been shown to apply to Galactic stars, especially those more massive than the Sun [72]. We choose this distribution for simplicity, as the true mass distribution for isolated black holes is unknown. In applying the Salpeter function to black holes, we implicitly ignore effects such as mass loss due to stellar winds, which has a larger effect on heavier stars. To better approximate the true mass distribution, we introduce minimum and maximum black hole masses for the population based on both observational and theoretical arguments. We use the same mass distribution for both the bulge and the disk [73], and we assume that it is unchanged over the lifetime of the Galaxy [72].

Two populations of stellar-mass black holes have been detected. All known Galactic black holes reside in binaries, and most are observable in X-rays due to accreting material 

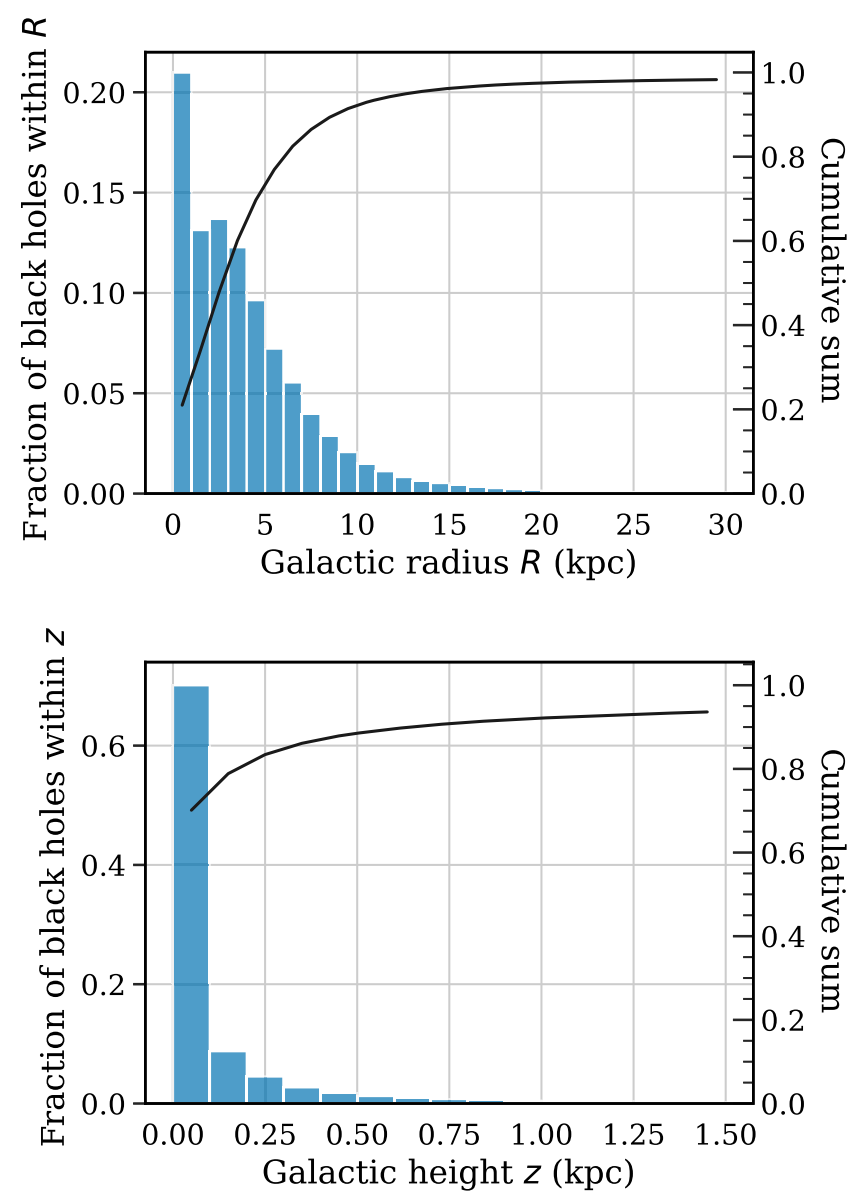

FIG. 8. Most black holes in our simulation are located close to the Galactic Center and at low Galactic latitudes. These regions tend to be metal rich, which can limit the maximum black hole mass $M_{\mathrm{BH} \text {,max }}$ that can form from stars.

from their stellar companions; these black holes tend to have masses $\lesssim 20 \mathrm{M}_{\odot}[16,17,74]$ (with the possible exception of LB-1, which has been claimed to have a mass of $70 \mathrm{M}_{\odot}$ [75], although this is controversial [76,77]). In contrast, the mass distribution of black holes in merging binaries detected by LIGO and Virgo is consistent with a mass cutoff of approximately $M_{\mathrm{BH}} \sim 40 \mathrm{M}_{\odot}$ and a powerlaw index of around 2 [78-80]; however, it is difficult to extrapolate from the properties of the gravitationally detected black holes, as these are found in other Galaxies with unknown star formation histories and metallicities. Indeed, studies suggest that low metallicity environments are required to produce these more massive black holes $[81,82]$. In contrast, most of the black holes in our simulation are at low Galactic latitudes and close to the Galactic Center (Fig. 8), where stars are metal rich [83,84], although the metallicity could have been different in the past [85].

With these considerations in mind, we take $\left[5 \mathrm{M}_{\odot}, 20 \mathrm{M}_{\odot}\right]$ as the standard distribution of black hole masses (Table I). This range includes the known Galactic black holes.
TABLE I. We consider five black hole populations. The black hole mass $M_{\mathrm{BH}}$ is drawn from an $M^{-2.35}$ distribution with the minimum and maximum masses given by the second column (Sec. III B). The initial black hole spin $\chi_{i}$ is drawn from a uniform distribution with limits given by the third column (Sec. III C).

\begin{tabular}{lcc}
\hline \hline & $M_{\mathrm{BH}}$ & $\chi_{i}$ \\
\hline Standard population & {$\left[5 \mathrm{M}_{\odot}, 20 \mathrm{M}_{\odot}\right]$} & {$[0,1.0]$} \\
Heavy population & {$\left[5 \mathrm{M}_{\odot}, 30 \mathrm{M}_{\odot}\right]$} & {$[0,1.0]$} \\
Moderate spins & {$\left[5 \mathrm{M}_{\odot}, 20 \mathrm{M}_{\odot}\right]$} & {$[0,0.5]$} \\
Heavy with moderate spins & {$\left[5 \mathrm{M}_{\odot}, 30 \mathrm{M}_{\odot}\right]$} & {$[0,0.5]$} \\
Pessimistic spins & {$\left[5 \mathrm{M}_{\odot}, 20 \mathrm{M}_{\odot}\right]$} & {$[0,0.3]$} \\
\hline \hline
\end{tabular}

A maximum mass of $20 \mathrm{M}_{\odot}$ is also consistent with simulations, which suggest that the heaviest black hole that can form at Galactic metallicities is around $20 \mathrm{M}_{\odot}$ [82].

Our results are not sensitive to the choice of minimum black hole mass for all but the heaviest boson masses; the loudest signals come from the heavier black holes, and bosons heavy enough to produce large strains around light black holes source few observable signals. See Appendix C for more discussion on the effect of decreasing the minimum black hole mass.

The choice of maximum black hole mass more directly impacts our simulated signals, as both the signal strength and duration are highly sensitive to the black hole mass. Since the loudest signals come from systems with the heaviest black holes, our choice of $20 \mathrm{M}_{\odot}$ is conservative. To evaluate the dependence of our conclusions on the maximum mass, we also consider heavy distributions with a maximum mass of $30 \mathrm{M}_{\odot}$ (Table I).

\section{Spin magnitude and orientation}

The strain observed at the detector will in general be smaller than the characteristic strain, $h_{0}$, by a factor that depends on the geometry of the detector and the source. Relevant parameters are the inclination angle $\imath$ (or, more directly, $\cos \imath$ ) and the polarization angle $\theta$, defined by the orientation of the spin axis of the black hole. We assume that the black hole spin axis is primarily determined by the angular momentum axis of the progenitor star. Since there is no known favored spin direction of stars in the Galaxy, we choose $\cos l$ and $\theta$ to be uniformly distributed in $[-1,1]$ and $[-\pi / 4, \pi / 4]$, respectively. We take $\phi_{0}$ (the phase of the gravitational wave at a chosen reference time) to be uniformly distributed in $[0,2 \pi]$.

The distribution of black hole spin magnitudes at birth is not well understood; in particular, there is no observational data for the spins of isolated Galactic black holes. Future analysis on the detectability of the boson ensemble signal in binaries would be interesting, as many high-spin channels are thought to occur in binary systems. Some 1D stellar evolution models indicate that a majority of black holes tend to be born with minimal spins (see, e.g., [86]); this is also compatible with the measurements of spins of pulsars 
extrapolated to their natal spins $[16,87]$. Models also predict a potentially small fraction (of the order of $10^{-3}-10^{-2}$ ) of black holes to be born with very high spins $(\chi>0.9)$ through the chemically homogeneous channel, thought to be associated with gamma-ray bursts $[88,89]$.

On the other hand, there are measurements of black hole spins in binary systems. Black holes in binaries observed to merge in LIGO have tended to low spins, although the error on measurements of initial spins is quite large; the inferred spin magnitudes of $90 \%$ of black holes are found to be below $0.6_{-0.28}^{+0.24}$ or $0.8_{-0.24}^{+0.15}$ in the aligned or isotropic spin scenarios, respectively [79], and typical spin magnitudes have a preferred low central value of $\sim 0.2 \pm 0.2[80,90]$. Black holes in X-ray binary systems have a range of spin values, from low to $\chi>0.9$, with order half with spin above $0.5[16,17,74]$. While accretion can contribute to the spinup of a black hole, the stellar companions in most of these systems are not long lived or not massive enough to substantially increase the black hole spin from its natal spin $[16,17,91]$.

Given these uncertainties and range of observations, we take a distribution uniform in the range $[0,1]$ as a standard and $\chi_{i, \max }=0.5,0.3$ as moderate and pessimistic spin distributions, respectively (Table I). We also discuss our conclusions in the case in which a subpopulation of black holes has moderate or high spin.

\section{Frequency derivatives}

Any astrophysical signal has a small apparent $\dot{f}$ due to the radial velocity relative to the observer changing over time, and thus a time-dependent Doppler shift, $\dot{f}_{\text {app }} \simeq \frac{\Delta v_{\text {rad }}}{c \Delta t} f$, where $v_{\text {rad }}$ is the radial velocity of the source and $d$ is the distance to the source. For distances that are large compared to the distance the black hole travels over the observational period, as is the case here, the change in the radial velocity can be related to the velocity tangential to the line of sight $v_{\tan }$ and distance to the source $d$ to give $\dot{f}_{\text {app }} \approx v_{\text {tan }}^{2} f / c d$. At the highest frequencies, $\dot{f}_{\text {app }}<$ $10^{-19} \mathrm{~Hz} / \mathrm{s}$ for the black hole population.

As discussed in Sec. II B, the decreasing mass of the boson cloud also causes a small spin-up. For a given $M_{\mathrm{BH}}$, the magnitude of $\dot{f}$ is largest for large values of $\mu_{\mathrm{b}}$. For the detectable systems in our search, the frequency derivatives fall in the range $10^{-14} \mathrm{~Hz} / \mathrm{s} \lesssim \dot{f}<6 \times 10^{-13} \mathrm{~Hz} / \mathrm{s}$ for both the standard and heavy black hole populations (Fig. 23).

These $\dot{f}$ values are smaller than the minimum $|\dot{f}|$ sensitivity of a typical semicoherent all-sky search. In general, an all-sky search for the boson annihilation signal does not need to include frequency derivatives. On the other hand, follow-up searches of interesting signal candidates may need to take $\dot{f}$ into account and could detect a small positive frequency drift, pointing toward the signal origin being a boson cloud.

\section{E. Systems outside the Milky Way}

We have focused on the signal from Galactic black holes, as they produce the loudest signals. We can also ask whether the black holes in the closest neighboring Galaxy, Andromeda, will produce a detectable signal. The distance to Andromeda is around $770 \mathrm{kpc}$ [92]. The signal strains would drop by almost 3 orders of magnitude compared to what is plotted in Fig. 3 so that systems in the top right corner of the plot (roughly speaking, $\mu_{\mathrm{b}}>1.4 \times 10^{-12} \mathrm{eV}$ and $M_{\mathrm{BH}}>15 \mathrm{M}_{\odot}$ would produce detectable signals. These systems correspond to $\alpha \gtrsim 0.17$ (Fig. 1), which have timescales of $\tau_{\mathrm{GW}}<10^{4} \mathrm{yr}$ (Fig. 4). It is therefore unlikely for annihilation signals from Andromeda to be detectable by the current generation of detectors.

Closer to home, the Large Magellanic Cloud (LMC) and the Small Magellanic Cloud (SMC) are potential sources of signals. The contribution from either of these two dwarf galaxies would be an additional overdensity of signals in a particular part of the sky and within a small range of Doppler shifts, since the black holes are traveling at the respective galaxy's bulk velocity $(68 \mathrm{~km} / \mathrm{s}$ for the LMC and $5 \mathrm{~km} / \mathrm{s}$ for the SMC [93]). The LMC and SMC are an order of magnitude further than the Galactic Center [94,95], so the peak strains are an order of magnitude below the peak of the Milky Way distribution. Since the expected signal is small and the star formation histories of the LMC and SMC are very uncertain, we do not include them in our simulations.

\section{THE ENSEMBLE SIGNAL}

In this section, we consider the ensemble of signals produced by annihilations of bosons with masses in the range $1 \times 10^{-13}$ to $4 \times 10^{-12} \mathrm{eV}$, in clouds around Galactic isolated black holes. To facilitate reproducibility and further studies, we provide the full set of simulated signal populations in an online repository [96].

We focus here on the standard black hole population (Table I).

\section{A. Signal simulation procedure}

Each of the $10^{8}$ black holes is tagged with a unique position, velocity, and age. For a given run (i.e., choice of $\mu_{\mathrm{b}}$ ), we randomly assign a mass and spin to each black hole as described in Secs. III B and III C and summarized in Table I. For each boson-black hole system, we compare the initial spin $\chi_{i}$ to the critical spin $\chi_{c}$ [Eq. (4)] to determine whether or not the cloud forms. If $\chi_{i}>\chi_{c}$, we calculate the instability timescale $\tau_{\text {inst }}$ [Eq. (5)] which defines the $e$-folding time for the cloud to grow, and we take $\ln (N) \tau_{\text {inst }} \approx 180 \tau_{\text {inst }}$ as the timescale for the cloud to reach its maximum size (as discussed in Sec. II A), keeping systems for which this time is less than the black hole age.

To check whether the second level has fully formed and therefore caused the continuous wave emission from the 
TABLE II. Representative values of relevant parameters and timescales for a selection of boson and black hole masses of interest. All black holes in the table possess initial spins of $\chi_{i}=0.9$. The dominant effect of $\chi_{i}$ is the determination of whether or not the cloud forms; the approximate formulations for the cloud and gravitational-wave parameters have linear dependencies on $\chi_{i}$. The listed values of $\alpha$ and $\chi_{c}$ correspond to the initial values at the time of black hole formation. $\tau_{\mathrm{GW}}$ is the decay rate of the gravitational-wave signal strength from its peak $h_{0, \text { peak }}$, while $\tau_{\text {inst }}^{022}$ is a strict cutoff on any gravitational-wave emission $\left(h_{0}=0\right.$ at $\left.t=\tau_{\text {inst }}^{022}\right)$.

\begin{tabular}{|c|c|c|c|c|c|c|c|c|}
\hline$\mu_{\mathrm{b}}(\mathrm{eV})$ & $M_{\mathrm{BH}}\left(\mathrm{M}_{\odot}\right)$ & $\alpha_{i}$ & $\chi_{c, i}$ & $\tau_{\text {inst }}(\mathrm{yr})$ & $\tau_{\text {inst }}^{022}(\mathrm{yr})$ & $h_{0, \text { peak }}$ at $1 \mathrm{kpc}$ & $\tau_{\mathrm{GW}}(\mathrm{yr})$ & $f_{\mathrm{GW}}(\mathrm{Hz})$ \\
\hline \multirow{4}{*}{$2 \times 10^{-13}$} & 5 & 0.00749 & 0.030 & $5.3 \times 10^{10}$ & $5.9 \times 10^{23}$ & $6.4 \times 10^{-32}$ & $3.2 \times 10^{21}$ & 96.72 \\
\hline & 10 & 0.0150 & 0.060 & $2.2 \times 10^{8}$ & $7.9 \times 10^{19}$ & $1.5 \times 10^{-29}$ & $2.3 \times 10^{17}$ & 96.72 \\
\hline & 20 & 0.0300 & 0.12 & $1.0 \times 10^{6}$ & $1.1 \times 10^{16}$ & $3.2 \times 10^{-27}$ & $1.9 \times 10^{13}$ & 96.71 \\
\hline & 30 & 0.0449 & 0.18 & $4.5 \times 10^{4}$ & $6.8 \times 10^{13}$ & $6.8 \times 10^{-26}$ & $8.7 \times 10^{10}$ & 96.70 \\
\hline \multirow{4}{*}{$5 \times 10^{-13}$} & 5 & 0.0187 & 0.075 & $1.5 \times 10^{7}$ & $1.8 \times 10^{18}$ & $3.4 \times 10^{-29}$ & $4.3 \times 10^{15}$ & 241.8 \\
\hline & 10 & 0.0374 & 0.15 & $7.2 \times 10^{4}$ & $2.7 \times 10^{14}$ & $6.9 \times 10^{-27}$ & $3.9 \times 10^{11}$ & 241.8 \\
\hline & 20 & 0.0749 & 0.29 & $4.1 \times 10^{2}$ & $4.6 \times 10^{10}$ & $1.2 \times 10^{-24}$ & $4.7 \times 10^{7}$ & 241.7 \\
\hline & 30 & 0.112 & 0.43 & $2.4 \times 10^{1}$ & $3.1 \times 10^{8}$ & $2.0 \times 10^{-23}$ & $2.9 \times 10^{5}$ & 241.5 \\
\hline \multirow{4}{*}{$8 \times 10^{-13}$} & 5 & 0.0300 & 0.12 & $2.5 \times 10^{5}$ & $2.8 \times 10^{15}$ & $7.9 \times 10^{-28}$ & $4.7 \times 10^{12}$ & 386.8 \\
\hline & 10 & 0.0599 & 0.24 & $1.3 \times 10^{3}$ & $4.6 \times 10^{11}$ & $1.4 \times 10^{-25}$ & $5.1 \times 10^{8}$ & 386.7 \\
\hline & 20 & 0.120 & 0.45 & $9.6 \times 10^{0}$ & $8.8 \times 10^{7}$ & $1.8 \times 10^{-23}$ & $9.4 \times 10^{4}$ & 386.3 \\
\hline & 30 & 0.180 & 0.63 & $7.9 \times 10^{-1}$ & $6.1 \times 10^{5}$ & $2.7 \times 10^{-22}$ & $7.1 \times 10^{2}$ & 385.5 \\
\hline \multirow{4}{*}{$1.5 \times 10^{-12}$} & 5 & 0.056 & 0.22 & $1.1 \times 10^{3}$ & $5.4 \times 10^{11}$ & $4.7 \times 10^{-26}$ & $6.3 \times 10^{8}$ & 725.1 \\
\hline & 10 & 0.112 & 0.43 & $7.8 \times 10^{0}$ & $1.0 \times 10^{8}$ & $6.7 \times 10^{-24}$ & $9.6 \times 10^{4}$ & 724.4 \\
\hline & 20 & 0.225 & 0.74 & $1.4 \times 10^{-1}$ & $2.0 \times 10^{4}$ & $5.7 \times 10^{-22}$ & $2.7 \times 10^{1}$ & 721.2 \\
\hline & $30^{*}$ & 0.34 & 0.92 & & & & & \\
\hline \multirow{4}{*}{$2.0 \times 10^{-12}$} & 5 & 0.0749 & 0.29 & 10. $\times 10^{1}$ & $1.1 \times 10^{10}$ & $2.9 \times 10^{-25}$ & $1.2 \times 10^{7}$ & 966.6 \\
\hline & 10 & 0.150 & 0.55 & $9.1 \times 10^{-1}$ & $2.3 \times 10^{6}$ & $2.9 \times 10^{-23}$ & $3.3 \times 10^{3}$ & 964.7 \\
\hline & 20 & 0.299 & 0.88 & $7.1 \times 10^{-2}$ & $3.4 \times 10^{2}$ & $7.9 \times 10^{-22}$ & $2.5 \times 10^{0}$ & 956.0 \\
\hline & $30^{*}$ & 0.449 & 0.99 & & & & & \\
\hline \multirow{4}{*}{$3.5 \times 10^{-12}$} & 5 & 0.131 & 0.49 & $1.2 \times 10^{0}$ & $6.6 \times 10^{6}$ & $7.6 \times 10^{-24}$ & $7.4 \times 10^{3}$ & 1689 \\
\hline & 10 & 0.262 & 0.82 & $3.4 \times 10^{-2}$ & $1.2 \times 10^{3}$ & $4.9 \times 10^{-22}$ & $2.5 \times 10^{0}$ & 1679 \\
\hline & $20^{\dagger}$ & 0.52 & & & & & & \\
\hline & $30^{\dagger}$ & 0.79 & & & & & & \\
\hline
\end{tabular}

${ }^{*} \chi_{i}<\chi_{c, i}$. The cloud does not form in this scenario.

$\alpha>0.49$. The cloud does not form in this scenario.

first level to cease, we also calculate $\tau_{\text {inst }}^{022}$ for the $(n=0$, $\ell=m=2$ ) level; for systems with black holes older than $\ln \left(N_{022}\right) \tau_{\text {inst }}^{022}$, we set $h_{0}=0$ since the first level is no longer radiating.

For systems that pass these checks, we calculate $h_{0 \text {,peak }}$ and $\tau_{\mathrm{GW}}$ [Eqs. (A17) and (A19)] and use the time evolution of $h_{0}$ [Eq. (14)] to determine its current value. The relevant time $t$ is the time since cloud formation minus the time $d / c$ required for the GW emission to travel the distance $d$ to Earth; if the emission since cloud formation has not yet reached Earth, we set $h_{0}=0$. We compute the frequency $f_{\mathrm{GW}}$ at cloud formation [Eq. (9)] and apply the frequency drift [Eq. (16)] over the lifetime of the cloud, which is the black hole age minus $\ln (N) \tau_{\text {inst }}$. Finally, we determine the apparent signal frequency by applying a Doppler shift

$$
f_{\mathrm{GW}, \mathrm{obs}}=\left(1-\frac{v_{\mathrm{rad}}}{c}\right) f_{\mathrm{GW}} \approx\left(1 \pm 10^{-4}\right) f_{\mathrm{GW}},
$$

where $f_{\mathrm{GW}, \text { obs }}$ is the observed gravitational-wave frequency due to the source's radial velocity as measured at the SSB. The maximum value of $\left|v_{\text {rad }} / c\right|$ for our black hole population is 0.0025 , and $90 \%$ of black holes have values of $\left|v_{\text {rad }} / c\right|<6 \times 10^{-4}$.

In Table II, we list a set of example systems representative of the parameter space on which this study focuses.

\section{B. Ensemble signal properties}

\section{Ensemble signal shape}

The source-frame frequency of the GW signal, $f_{\mathrm{GW} \text {,source }}$, has a small dependence on $M_{\mathrm{BH}}$ through $\alpha$, resulting in different "potential energy" corrections for different black hole masses. For a fixed boson mass, increasing the black hole mass produces a signal with a lower frequency [Eq. (10)] and a higher peak strain [Eq. (13)]. Thus, in a given ensemble, the signals with lower frequencies have higher strains at cloud formation. The signal half-time $\tau_{\mathrm{GW}}$ decreases rapidly with increasing black hole mass [Eq. (15)]. If $\tau_{\mathrm{GW}}$ is shorter than the black hole age $\tau_{\mathrm{BH}}$, the signal strain today is suppressed by $\tau_{\mathrm{GW}} / \tau_{\mathrm{BH}}$; increasing the black hole mass above the value that gives $\tau_{\mathrm{GW}} \sim \tau_{\mathrm{BH}}$ increases the peak strain but decreases 

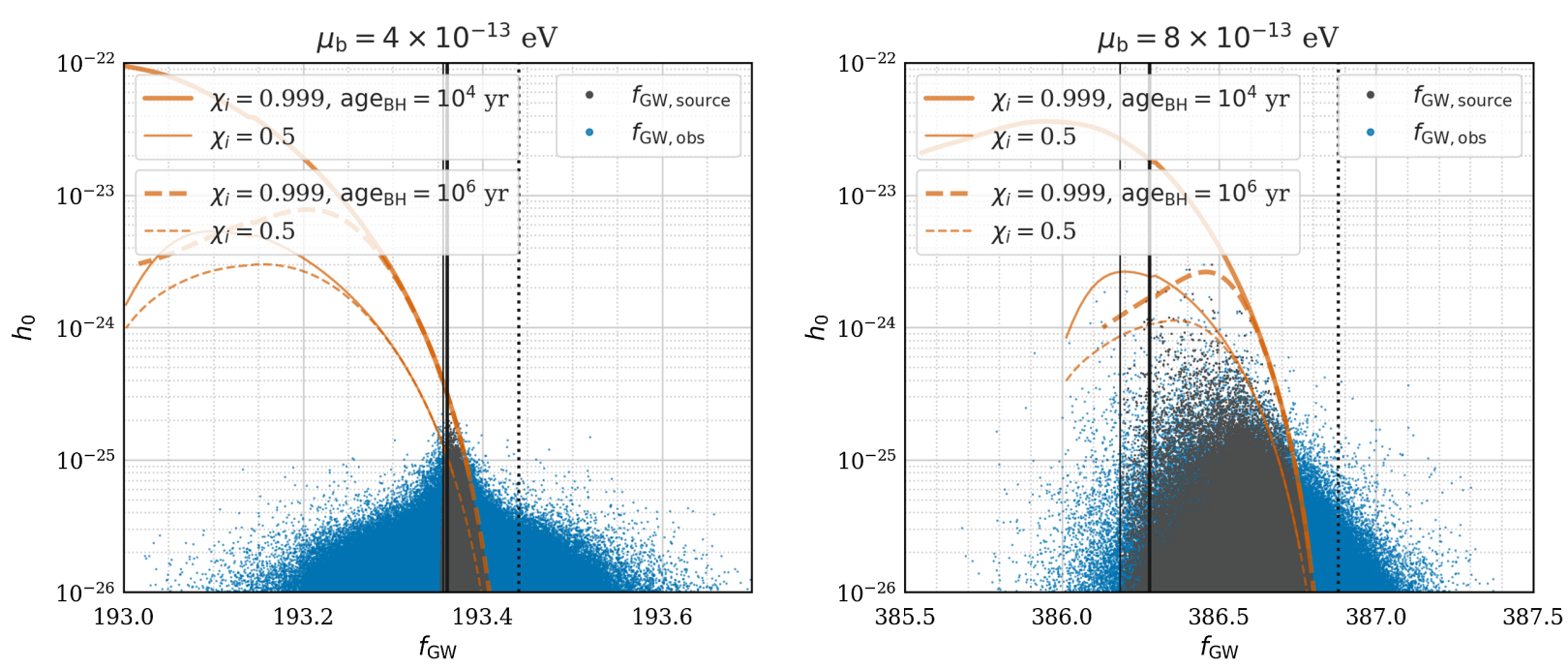

FIG. 9. The distribution of signal strains as a function of source frequency (gray dots) and observed frequency with Doppler shift (blue dots). The strains are selected for signals with $d \geq 1 \mathrm{kpc}$ to facilitate comparison to the strain as a function of source frequency for a system at a distance $d=1 \mathrm{kpc}$ : the upper envelope for two sets of black hole spin and age parameters is shown in orange. The black vertical lines show the boundaries of the source frequency distribution: the vertical dotted line shows the rest mass frequency $f_{\mathrm{GW}}^{0}$, and the thick and thin vertical lines correspond to $f_{\mathrm{GW} \text {,source }}$ for $\left(20 \mathrm{M}_{\odot}, \chi_{i}=0.999\right)$ and $\left(20 \mathrm{M}_{\odot}, \chi_{i}=0.5\right)$, respectively, yielding successively more negative potential energy corrections. (In the left panel, the two solid vertical lines lie close to each other.)

the strain at the current time. The current strain as a function of frequency for a fixed black hole age and distance as seen in the source frame is shown in the orange curves of Fig. 9.
The maximum frequency in an ensemble in the source frame is $f_{\mathrm{GW}}^{0}$, while the minimum value depends on multiple factors: there are no signals below a certain source frequency if (a) there are no heavier black holes due to the
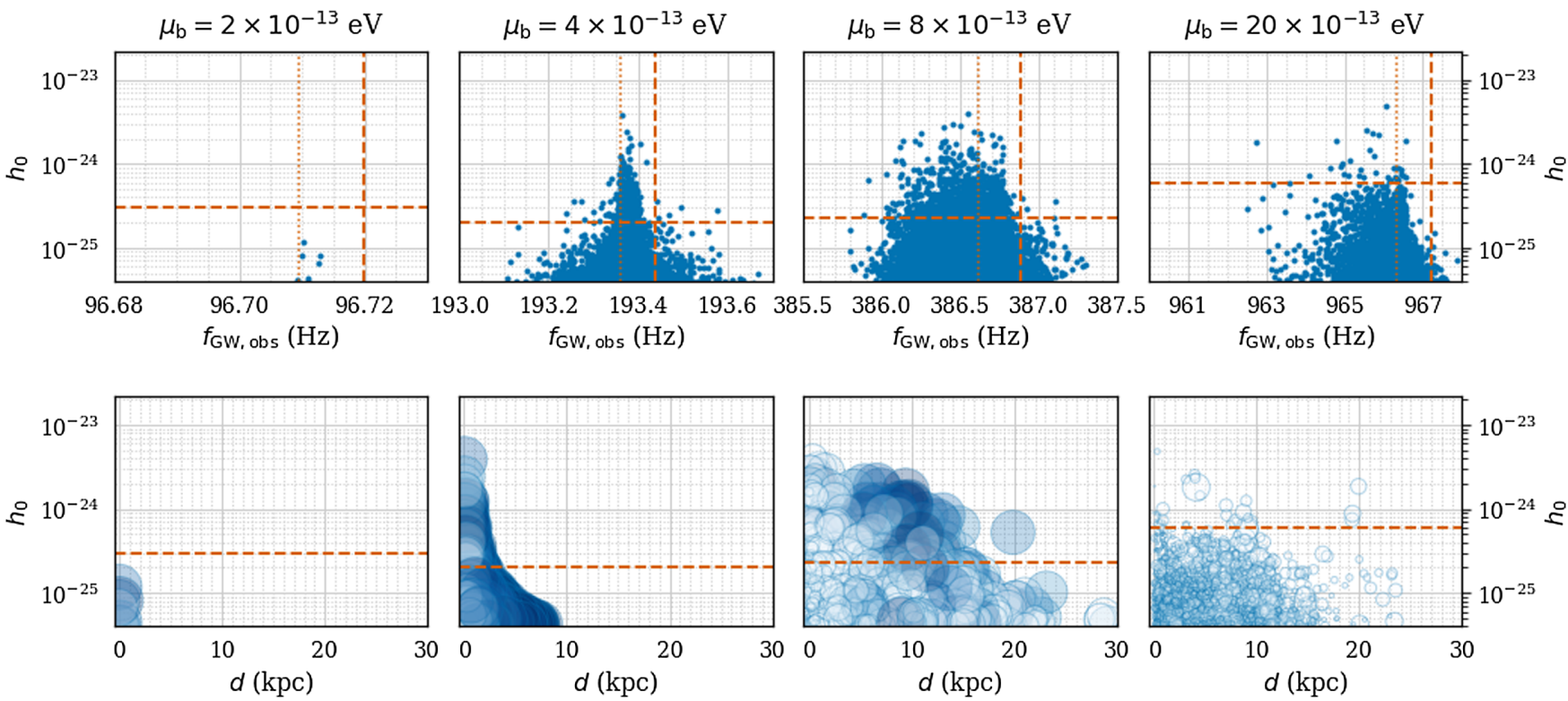

FIG. 10. The ensemble signals for the "standard" black hole population (Table I) and boson mass (left to right) $\mu_{\mathrm{b}}=2 \times 10^{-13}, 4 \times 10^{-13}, 8 \times 10^{-13}$, and $20 \times 10^{-13} \mathrm{eV}$. Top row: Each point represents the annihilation signal from an individual system, illustrating the range of strains and frequencies of the signals. The dashed horizontal red line corresponds to the approximate upper limit at that frequency reported by recent all-sky continuous wave searches $[20,21,47,97]$. The thick dashed vertical red line marks the values of $f_{\mathrm{GW}}^{0}$, and the dotted red line shows $f_{\mathrm{GW}}$ calculated using the mode of the $M_{\mathrm{BH}}$ distribution (Fig. 11). Bottom row: Distribution of strains as a function of distance and black hole mass. Smaller, whiter circles correspond to lighter black holes (minimum $5 \mathrm{M}_{\odot}$ ) and larger bluer circles correspond to heavier black holes (maximum $20 \mathrm{M}_{\odot}$ ); the differences in circle sizes are exaggerated for visual effect and do not scale linearly with $M_{\mathrm{BH}}$. The horizontal dashed red line again represents current search upper limits. 

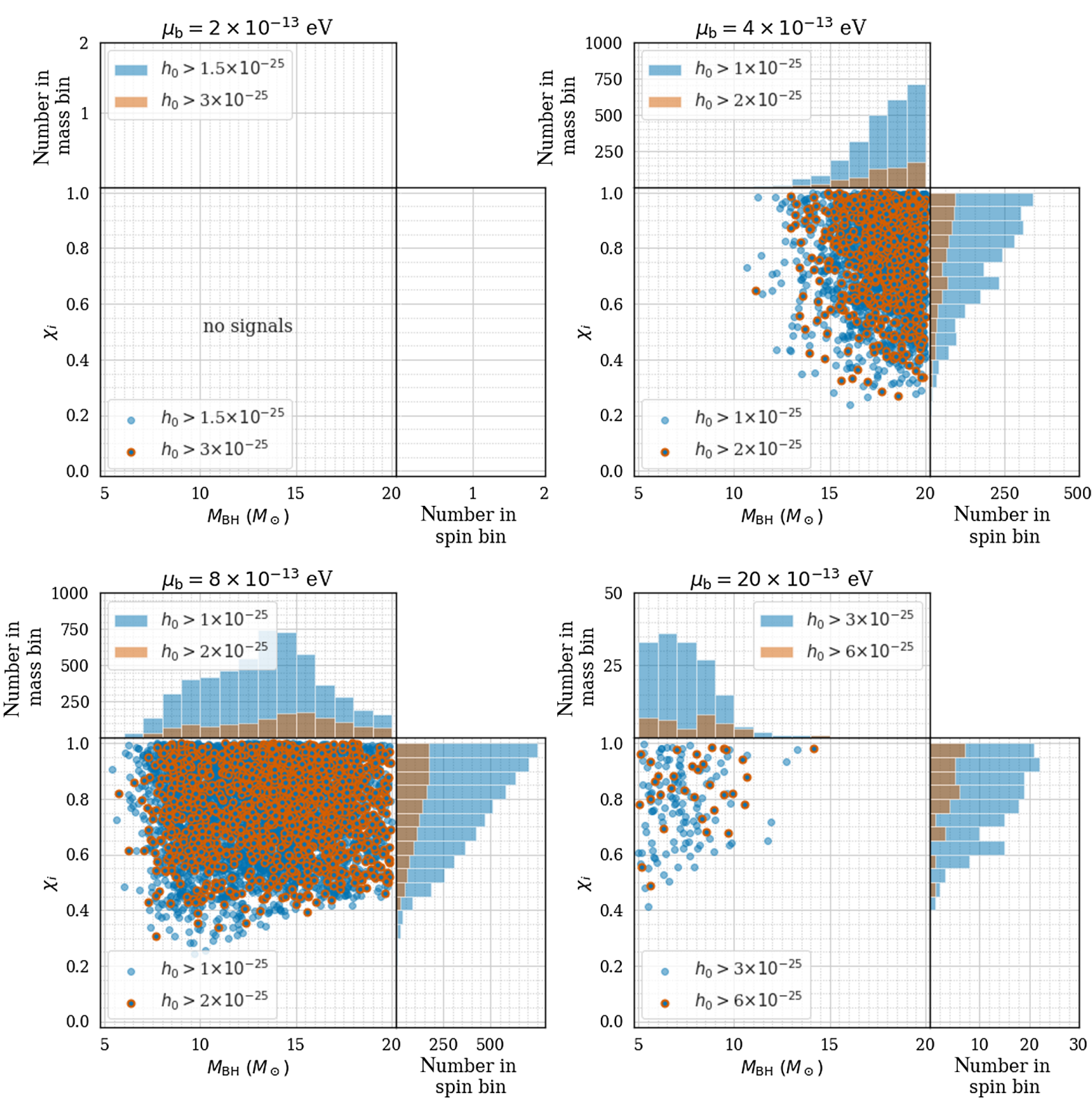

FIG. 11. Each of the four panels shows the mass $M_{\mathrm{BH}}$ and initial spin $\chi_{i}$ of the black holes that produce the ensemble signals for the four boson masses from Fig. 10, assuming the standard black hole population (Table I). In each panel, the scatterplot shows $M_{\mathrm{BH}}$ and $\chi_{i}$ for the systems that are detectable with the current search sensitivities (blue circles with orange outlines) and a factor of 2 improvement in sensitivity (blue circles). The histograms show the distributions of $M_{\mathrm{BH}}$ (top panels) and $\chi_{i}$ (right panels) for these sets of systems. The black holes are assigned spins uniformly distributed in [0, 1], and only systems satisfying Eq. (4) will form clouds. The upper-left set of plots $\left(\mu_{\mathrm{b}}=2 \times 10^{-13} \mathrm{eV}\right)$ contains no signals at these values of $h_{0}$, suggesting that a larger increase in sensitivity is necessary to detect the annihilation signals produced by the lightest bosons.

imposed maximum black hole mass cutoff, (b) heavier black holes result in prohibitively large critical spins $\chi_{c}$ [Eq. (4)] and hence systems with heavier black holes never form, or (c) heavier black holes have short superradiance times for the $\ell=m=2$ level, $\tau_{\text {inst }}^{022}$ [Eq. (A7)], cutting off the signal before today. The ensemble-signal shape as seen in the source frame is shown by the gray dots in Fig. 9.

The line-of-sight black hole velocity produces an additional Doppler shift for each source, proportional to $v_{\mathrm{rad}} / c$, which "smears" the ensemble signal's distribution in frequency (the blue dots in Fig. 9). Nearby black holes produce stronger signals than faraway ones, while farther away black holes tend to have larger line-of-sight velocities (Fig. 7). This means that weaker (more distant) signals tend to have larger relative Doppler shifts. The result is that, at a fixed boson mass, the ensemble of signal frequencies observed at the detector $\left(f_{\mathrm{GW}, \mathrm{obs}}\right)$ is shaped roughly like a triangle, with a wider base and a central "peak." In Fig. 10 (top row), we show the ensemble signals from the standard population of $10^{8}$ black holes at a few illustrative boson masses. 
The spread in $f_{\mathrm{GW}, \text { obs }}$ is greater for heavier bosons for several reasons. First, heavier bosons produce gravitational-wave $(\mathrm{GW})$ emission with larger values of $f_{\mathrm{GW}}^{0}$, and as both the Doppler shift [Eq. (24)] and potential energy shift [Eq. (10)] terms are proportional to $f_{\mathrm{GW}}^{0}$, higher frequencies result in a larger absolute frequency spread, $\Delta f_{\mathrm{GW}}$. In addition, increasing the boson mass from $2 \times 10^{-13}$ to $8 \times 10^{-13} \mathrm{eV}$ increases the relative frequency spread, $\Delta f_{\mathrm{GW}} / f_{\mathrm{GW}}^{0}$, for two reasons: Within this mass range, (1) heavier bosons form systems with a larger range of black hole masses (Fig. 11), leading to a larger range of potential energy corrections [Eq. (10)], and (2) heavier bosons produce detectable signals at larger distances (Fig. 10, bottom row), resulting in larger Doppler corrections. Increasing the boson mass further starts to reduce these effects; the relative frequency spread decreases, while the absolute frequency spread continues to increase. In all cases, the signal frequencies lie in the range $f \in f_{\mathrm{GW}}^{0} \times\{1-0.01,1+0.0025\}$, where the upper end of the range is given by the maximum Doppler shift in the ensemble and the lower end of the range is given by the largest negative Doppler shift plus the largest observed potential energy correction with $\alpha \sim 0.25$.

\section{Maximum signal strength}

The power emitted by the boson cloud in gravitational waves is set by the total energy of the cloud, and the timescale $\tau_{\mathrm{GW}}$ over which the annihilations take place, $P \sim M_{\text {cloud }} / \tau_{\mathrm{GW}}$, providing an estimate of the maximum strain in the detector today:

$$
\begin{aligned}
h_{0} \lesssim & \left(\frac{5 G M_{\text {cloud }} / \tau_{\mathrm{GW}}}{2 \pi^{2} c f_{\mathrm{GW}}^{2} r^{2}}\right)^{1 / 2} \\
\lesssim & 10^{-23}\left(\frac{M_{\text {cloud }}}{0.05 M_{\mathrm{BH}}}\right)^{1 / 2}\left(\frac{M_{\mathrm{BH}}}{20 \mathrm{M}_{\odot}}\right)^{1 / 2}\left(\frac{4 \times 10^{-13} \mathrm{eV}}{\mu_{\mathrm{b}}}\right) \\
& \times\left(\frac{10^{5} \text { years }}{\tau_{\mathrm{GW}}}\right)^{1 / 2}\left(\frac{2 \mathrm{kpc}}{r}\right) .
\end{aligned}
$$

Here we have chosen near-maximal parameters for the cloud and black hole mass, as well as the lightest axion mass that produces observable signals; note that $M_{\text {cloud }}$ and $\tau_{\mathrm{GW}}$ are not independent parameters and cannot reach the optimal values for most $\left(\mu_{\mathrm{b}}, M_{\mathrm{BH}}\right)$ combinations. In addition, given the finite birth rate of black holes and their spatial distribution, the nearby black holes tend to be older, while the small number of young black holes are on average farther away (Fig. 12). We also see that black holes younger than $10^{5} \mathrm{yr}$ contribute to the potentially detectable signal for heavier bosons, but the strain is reduced due to the higher signal frequency and greater typical black hole distance. Thus, it is very unlikely to find strains larger than $h_{0} \sim 10^{-23}$. The typical distribution from black holes at $\gtrsim 1 \mathrm{kpc}$ as well as the analytical strain amplitudes for different black hole age and spin parameter choices are shown in Fig. 9.

\section{Properties of black holes in potentially detectable systems}

The detectability of a signal depends on the exact search involved. As a guideline, we take the approximate search sensitivities of recent all-sky searches in Advanced LIGO data [20-22] at the relevant frequencies.

\section{Black hole distances}

By construction, all the signals in the ensemble arise from black holes within the Milky Way (Fig. 12). The number of black holes increases rapidly as a function of distance, and in general many distant black holes produce signals below the typical search sensitivity (Fig. 10).

If the maximum black hole mass $M_{\mathrm{BH}, \max }=20 \mathrm{M}_{\odot}$, then for the lighter bosons, the signals are very weak and are observable only from short distances (e.g., less than $2 \mathrm{kpc}$ for $\mu_{\mathrm{b}}=4 \times 10^{-13} \mathrm{eV}$ ). Increasing the boson mass increases the signal power, and at $\mu_{\mathrm{b}}=8 \times 10^{-13} \mathrm{eV}$ signals at $10 \mathrm{kpc}$ are observable, although most signals are still produced by systems within $2 \mathrm{kpc}$. If we allow for $M_{\mathrm{BH} \text {,max }}=30 \mathrm{M}_{\odot}$, systems as far away as $15 \mathrm{kpc}$ are detectable for $\mu_{\mathrm{b}} \geq 8 \times 10^{-13} \mathrm{eV}$ (Fig. 34).

\section{Black hole masses}

We consider black holes with masses between $5 \mathrm{M}_{\odot}$ and $20 \mathrm{M}_{\odot}\left(30 \mathrm{M}_{\odot}\right)$ in our standard (heavy) population. For lighter bosons, the signals are weak and the distribution of black holes is therefore peaked toward the heavier black holes (Fig. 11). The signal strain increases with increasing black hole mass, $h_{0} \propto M_{\mathrm{BH}}^{8}$ [Eq. (13)], and the total number of signals is thus sensitive to the upper cutoff of the mass distribution. This is especially evident for $\mu_{\mathrm{b}}=2 \times 10^{-13} \mathrm{eV}$; while the standard population produces no signals with $h_{0}>1.5 \times 10^{-25}$ (Fig. 11), the heavy black hole population produces tens of such signals (Fig. 33).

In contrast, for heavier bosons, there are fewer signals from heavier black holes because of prohibitively high critical spins $\chi_{c}$ or short instability $\left(\tau_{\text {inst }}^{022}\right)$ and gravitationalwave $\left(\tau_{\mathrm{GW}}\right)$ timescales, as discussed in Sec. IV B. Because of these effects, for $\mu_{\mathrm{b}} \gtrsim 8 \times 10^{-13} \mathrm{eV}$, the fraction of signals with black hole masses of $20 \mathrm{M}_{\odot}$ or above goes to zero (Fig. 11).

The effect of the minimum black hole mass is relatively weak: we find that $5 \mathrm{M}_{\odot}-6 \mathrm{M}_{\odot}$ black holes do not contribute any signals above current search sensitivities for boson masses up to $8 \times 10^{-13} \mathrm{eV}$. Black holes as light as $5 \mathrm{M}_{\odot}$ can form systems with $\alpha>0.075$ for $\mu_{\mathrm{b}} \gtrsim 2 \times 10^{-12} \mathrm{eV}$, and they comprise $\sim 20 \%$ of the population of detectable signals at $\mu_{\mathrm{b}} \sim 2 \times 10^{-12} \mathrm{eV}$. Thus, decreasing $M_{\mathrm{BH} \text {,min }}$ would give a slightly larger number of signals for boson masses $\gtrsim 2 \times 10^{-12} \mathrm{eV}$. However, the instrument sensitivity is 

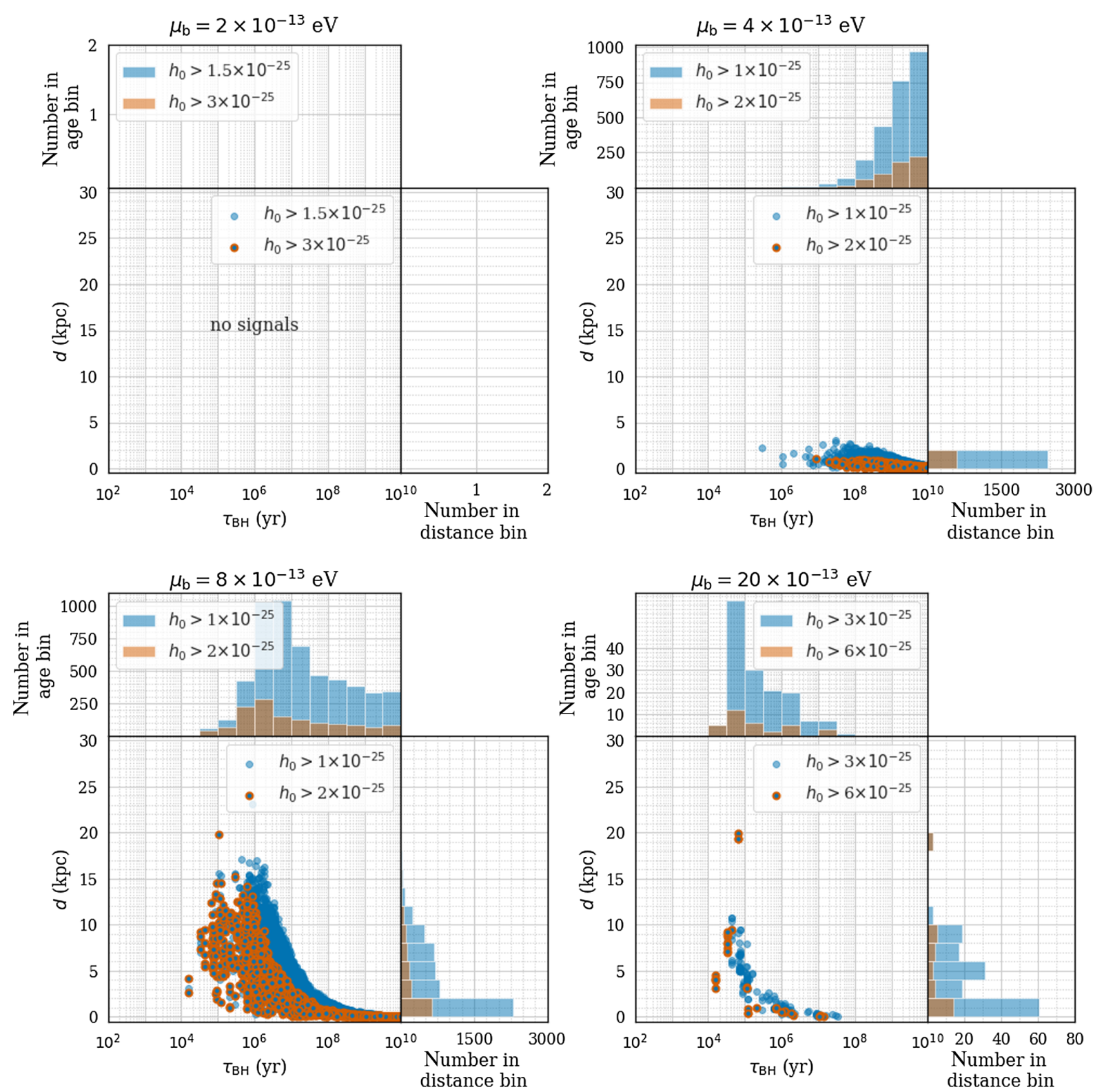

FIG. 12. Each of the four panels shows the age $\tau_{\mathrm{BH}}$ and distance $d$ of the black holes that produce the ensemble signals for the four boson masses in Fig. 10, assuming the standard black hole population (Table I). In each panel, the scatterplot shows $\tau_{\mathrm{BH}}$ and $d$ for the systems that are detectable with the current search sensitivities (blue circles with orange outlines) and a factor of 2 improvement in sensitivity (blue circles). The histograms show the distributions of $\tau_{\mathrm{BH}}$ (top panels) and $d$ (right panels) for these sets of systems. As in Fig. 11, the upper-left set of plots $\left(\mu_{\mathrm{b}}=2 \times 10^{-13} \mathrm{eV}\right)$ contains no signals at these values of $h_{0}$.

lower at the high frequencies corresponding to these heavier bosons, and few clouds are radiating today (Fig. 14).

\section{Black hole spins}

We find that only systems with initial spins of $\chi_{i}>0.25$ produce strains above current search sensitivities (Figs. 11 and 13). Systems with $\chi<0.25$ require $\alpha \lesssim 0.06$ for the cloud to form, producing signals that are too weak to observe. For $\mu_{\mathrm{b}} \geq 8 \times 10^{-13} \mathrm{eV}$, there is a trend toward higher black hole spins as the black hole mass increases (Fig. 11) due to the critical spin increasing for larger $\alpha$.
We show the number of signals with $h_{0}>10^{-25}$ as a function of $\chi_{i, \max }$ for six examples of boson masses in Fig. 13. The lightest boson mass produces few to no signals with $h_{0}>10^{-25}$; for the heavier five boson masses, the number of signals with $h_{0}>10^{-25}$ drops by at least an order of magnitude in reducing the maximum spin from $\chi_{i, \max }=$ 1.0 to $\chi_{i, \max }=0.5$, and 2 orders of magnitude when $\chi_{i, \max }$ decreases to 0.3 . For $\mu_{\mathrm{b}}=2 \times 10^{-12}$ and $3.5 \times 10^{-12} \mathrm{eV}$, there are no signals at or below $\chi_{i, \max }=0.3$ and 0.5 , respectively, since these critical spins would require black holes with mass below $5 \mathrm{M}_{\odot}$ to form clouds. 


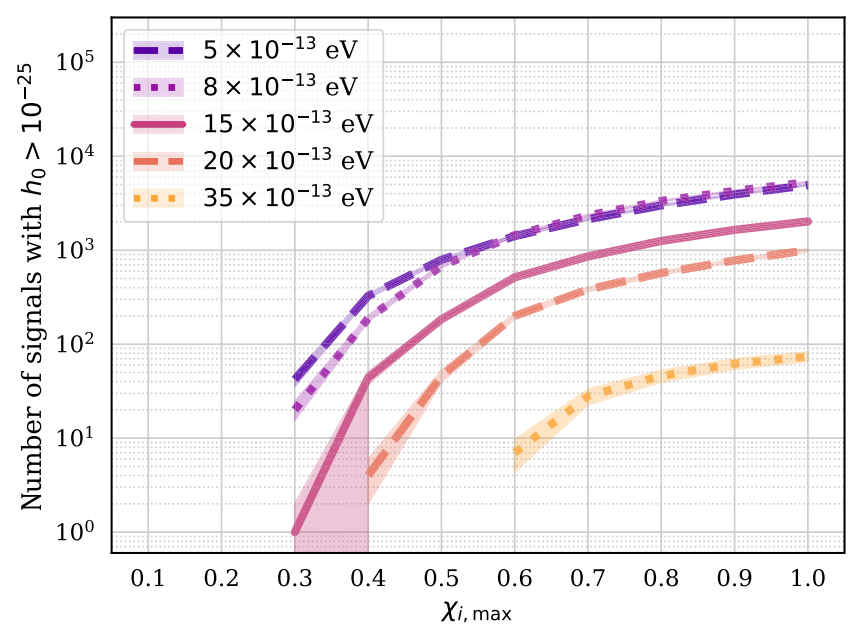

FIG. 13. The number of signals above $h_{0}>10^{-25}$ depends on the maximum of the initial spin distribution, $\chi_{i, \max }$. For a cloud to form, the initial spin must be larger than the critical spin [Eq. (4)]. Since $\chi_{c}$ increases with $\alpha$, this also results in fewer signals above a given strain for heavier bosons. The contours show Gaussian statistical uncertainties. There are no signals with amplitude $h_{0}>$ $10^{-25}$ for $\mu_{\mathrm{b}}=2 \times 10^{-13} \mathrm{eV}$ for any value of $\chi_{i, \max }$.

\section{Black hole ages}

Given the approximately constant-in-time black hole formation rate, young black holes are proportionally less common than old ones. For the lighter bosons, e.g., $\mu_{\mathrm{b}}=4 \times 10^{-13} \mathrm{eV}$, detectable signals arise from heavier black holes (Fig. 11), which are relatively rare; since black holes that are both heavy and young are doubly uncommon, the black holes that produce detectable signals for this boson mass are predominantly $10^{8}-10^{10}$ years old; Fig. 12. Bosons with $\mu_{\mathrm{b}}=8 \times 10^{-13} \mathrm{eV}$ produce detectable signals around black holes with masses across the entire range and have intermediate signal times, so these black holes span the entire range of ages. Finally, for the heaviest bosons, e.g., $\mu_{\mathrm{b}}=2 \times 10^{-12} \mathrm{eV}$, the shortness of the gravitational-wave timescale [Eq. (15)] and the instability timescale of the second level [Eq. (17)] means that very old systems have stopped radiating, so that only young black holes produce detectable signals.

As discussed in Sec. III A, our method of producing $10^{8}$ black holes results in fewer young black holes than the population would statistically contain. Specifically, considering our black hole formation rates, on average we expect $\mathcal{O}(10)$ black holes of $\tau_{\mathrm{BH}} \sim 10^{3} \mathrm{yr}$, and $\mathcal{O}(1)$ black holes of $\tau_{\mathrm{BH}} \sim 10^{2} \mathrm{yr}$. On the other hand, our sample population overestimates the number of $10^{4} \mathrm{yr}$ black holes, as the initial population included an upward fluctuation of the number of these black holes. Thus, our population includes 200 black holes that are younger than $10^{4}$ years old, compared to an average expectation of 62. This disproportionately affects heavier bosons; at $\mu_{\mathrm{b}}=2 \times$ $10^{-12} \mathrm{eV}$, of the order of half of the signals that are

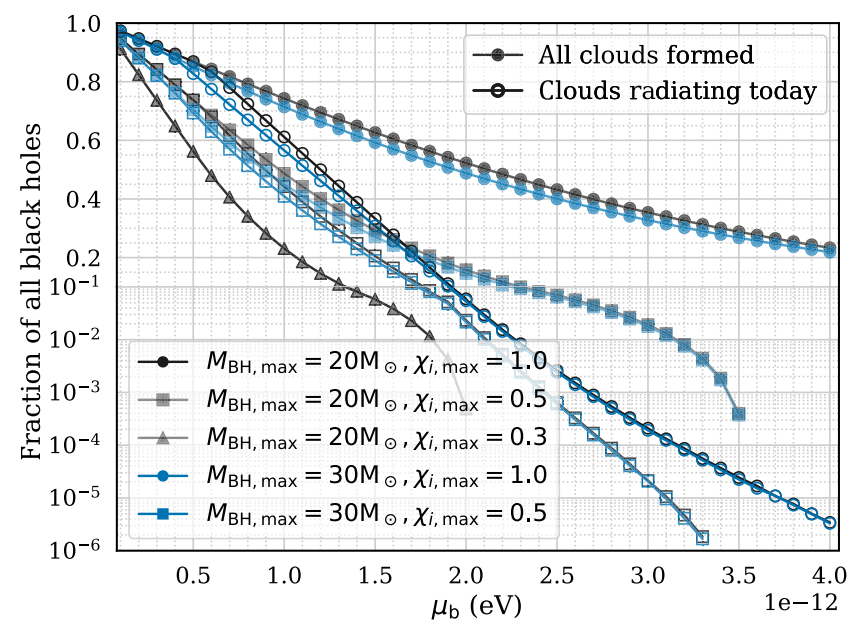

FIG. 14. For a given boson mass, a fraction of black holes is born with spins high enough to support cloud formation (filled markers). Gravitational-wave emission stops when the second level is fully populated, so a subset of the initial clouds are still emitting today (unfilled markers). We consider the five black hole populations of Table I: standard (black circles), heavy (blue circles), moderate spins (gray squares), heavy with moderate spins (blue squares), and pessimistic spin (gray triangles) populations. The percentage of systems still emitting today decreases rapidly with increasing boson mass; at $\mu_{\mathrm{b}}=4 \times 10^{-12} \mathrm{eV}$, only up to one in every $10^{6}$ black holes in the Galaxy is in a currently emitting system.

detectable with current search sensitivities are produced by $\sim 10^{4}$ year old black holes. Therefore, our sampling procedure introduces an order- 1 uncertainty in the number of signals produced by bosons with $\mu_{\mathrm{b}} \geq 2 \times 10^{-12} \mathrm{eV}$.

\section{Number and density of signals as a function of GW amplitude}

Figure 15 shows the total number of signals with characteristic strains above given values of $h_{0} \in\left[10^{-26}, 10^{-23}\right]$ for six reference boson masses. For most values of $h_{0}$, the number of signals above a given $h_{0}$ is highest for $\mu_{\mathrm{b}}=$ $8 \times 10^{-13} \mathrm{eV}\left(f_{\mathrm{GW}} \approx 400 \mathrm{~Hz}\right)$.

For the standard black hole population $\left(M_{\mathrm{BH}, \max }=\right.$ $20 \mathrm{M}_{\odot}$ ), annihilation signals from the lightest bosons are unlikely to be detectable by current continuous wave searches: for $\mu_{\mathrm{b}}=2 \times 10^{-13} \mathrm{eV}$ (corresponding to $f_{\mathrm{GW}} \sim 100 \mathrm{~Hz}$ ), few or no systems produce $\mathrm{GW}$ emission with intrinsic amplitude above $10^{-25}$. The loudest signal in our example has a strain a factor of a few below the current sensitivity thresholds (Fig. 10).

At fixed black hole mass, increasing the boson mass increases the strain as $\mu_{\mathrm{b}}^{9}$. Thus, increasing the boson mass by a factor of 2 to $\mu_{\mathrm{b}}=4 \times 10^{-13} \mathrm{eV}\left(f_{\mathrm{GW}} \approx 200 \mathrm{~Hz}\right)$ greatly increases the number of signals that would be detectable by current searches $\left(h_{0}>2 \times 10^{-25}\right.$ near this frequency). 


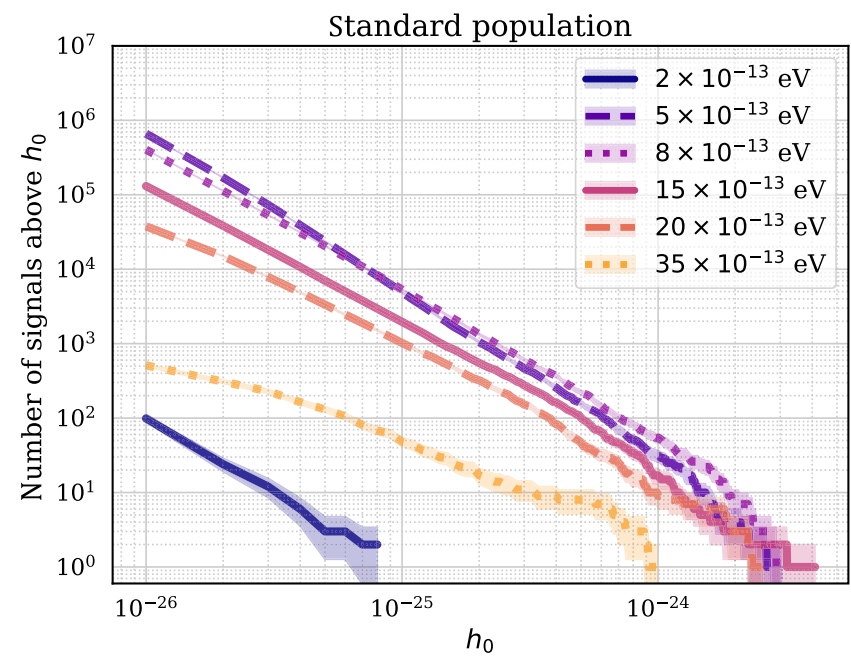

FIG. 15. The number of signals with intrinsic amplitude above a given $h_{0}$ value. The signal number is generally largest at $\mu_{\mathrm{b}}=$ $8 \times 10^{-13} \mathrm{eV}\left(f_{\mathrm{GW}} \approx 400 \mathrm{~Hz}\right)$ for the standard black hole population (Table I). At higher boson masses, the number of signals decreases because both the signal half-time [Eq. (15)] and the signal cutoff time due to the formation of the second level [Eq. (17)] decrease with increasing frequency. The contours show Gaussian statistical uncertainties, an underestimate at a small signal number.

Although the peak strain increases with boson mass, fewer signals are expected at heavier boson masses: Fig. 14 illustrates that both the percentage of systems with fully formed clouds and the percentage of systems that are still radiating continuous waves today decrease with boson mass. The further effect of the signal half-time is not included in Fig. 14 as it depends on the search strain sensitivity and the black hole's age. Figure 15 shows that for bosons with $\mu_{\mathrm{b}}>8 \times 10^{-13} \mathrm{eV}$, the number of signals above a given $h_{0}$ begins to decrease with increasing boson mass.

Figures 16 and 17 show the mean and maximum density of signals in frequency space above a characteristic strain $h_{0}$ for the same six reference bosons as in Fig. 15. Both the mean and maximum signal density are largest for $\mu_{\mathrm{b}}=5 \times 10^{-13} \mathrm{eV}$. This is in contrast to the fact that the number of signals is typically larger for $8 \times 10^{-13} \mathrm{eV}$ (Fig. 15). Signals from heavier bosons are spread over a larger frequency range as described in Sec. IV B, thereby decreasing the signal density.

Finally, Figs. 15-17 demonstrate how signal number and density increase with decreasing strain. The number of signals and the maximum signal density for a future search sensitivity an order of magnitude below the current continuous wave searches can be expected to increase by up to 2 orders of magnitude. Thus, we highlight that as the gravitational-wave observatories improve further continuous wave searches for bosons can be increasingly promising and less reliant on assumptions on black hole

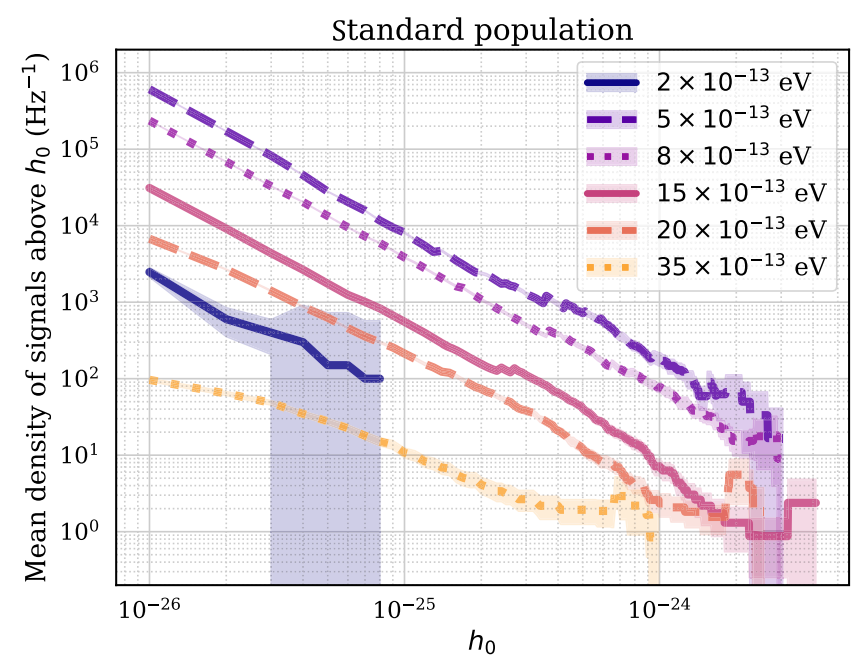

FIG. 16. The mean density of signals above a given $h_{0}$. The mean density is largest for $\mu_{\mathrm{b}}=5 \times 10^{-13} \mathrm{eV}\left(f_{\mathrm{GW}} \approx 250 \mathrm{~Hz}\right)$ for the standard black hole population (Table I). For heavier bosons, the overall density decreases; the number of signals above a given $h_{0}$ drops due to the combination of effects described in Fig. 15, while the frequency range spanned by the ensemble signal continues to increase. The contours show the uncertainty $\sqrt{N} / \Delta f$, where $N$ is the number of signals and $\Delta f$ is the frequency range spanned by the $N$ signals. Note that this does not take into account the additional uncertainty from $\Delta f$, which is itself correlated with $N$.

populations. On the other hand, the issues of signal detectability as detailed in the following section will only become more important to consider in the design and

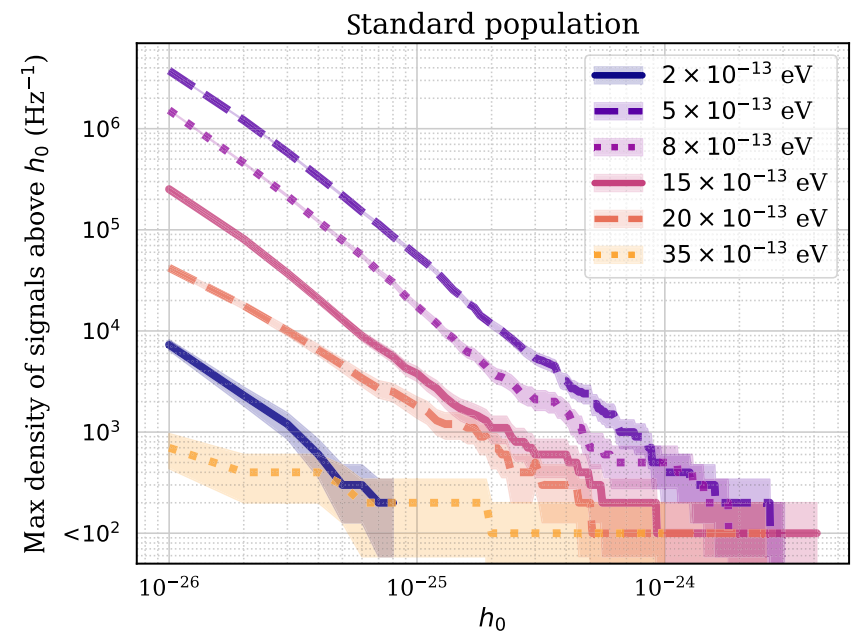

FIG. 17. The maximum density of signals above a given $h_{0}$ is up to an order of magnitude larger than the mean density (Fig. 16). Frequency ranges with sufficiently high signal densities can cause complications for standard searches for continuous wave signals (Sec. V). The contours show the uncertainty $\sqrt{N} / \Delta f$, where $N$ is the number of signals and $\Delta f=0.01 \mathrm{~Hz}$ is the size of the frequency bin over which the maximum density is calculated. 
interpretation of continuous wave searches for boson ensemble signals.

\section{SIGNAL DETECTABILITY}

We now consider the signal detectability in the context of the results of current continuous wave searches with LIGO data, using several black hole mass and spin distributions (Table I).

\section{A. Number of detectable signals}

For bosons in the mass range $\mu_{\mathrm{b}} \in\left[1 \times 10^{-13}\right.$, $\left.4 \times 10^{-12}\right] \mathrm{eV}$, in increments of $2 \times 10^{-14} \mathrm{eV}$, we calculate the number of detectable signals for the five black hole populations (Table I) at the corresponding frequencies $f_{\mathrm{GW}, \mathrm{obs}} \in[50,2000] \mathrm{Hz}$. For a signal to be detectable, we require that its intrinsic strain $h_{0}$ be greater than the $95 \%$ upper limit value in the $\left\{f_{\mathrm{GW}, \mathrm{obs}}\right\}$ frequency bin. Finally, we multiply the total number of detectable signals for each boson mass by $95 \%$.

We use the $95 \%$ upper limits for the low- $\dot{f}$ continuous wave search in the 20-600 Hz range [20,21] and the upper limits for the continuous wave search in the $10-2048 \mathrm{~Hz}$ range [22]. For ease of reading, we refer to the searches [20-22] by their nicknames "Falcon" and "Freq Hough," respectively. For bosons with $\mu_{\mathrm{b}}<1.2 \times 10^{-12} \mathrm{eV}$, we use the near-zero spin-down upper limits from the Falcon searches and for $\mu_{\mathrm{b}} \geq 1.2 \times 10^{-12} \mathrm{eV}$, we use the upper limits from the very broad spin-down Freq Hough search.

The number of detectable signals for the five black hole populations is shown in Fig. 18. All the curves peak at intermediate values of $\mu_{\mathrm{b}}$. As discussed in Secs. IV B

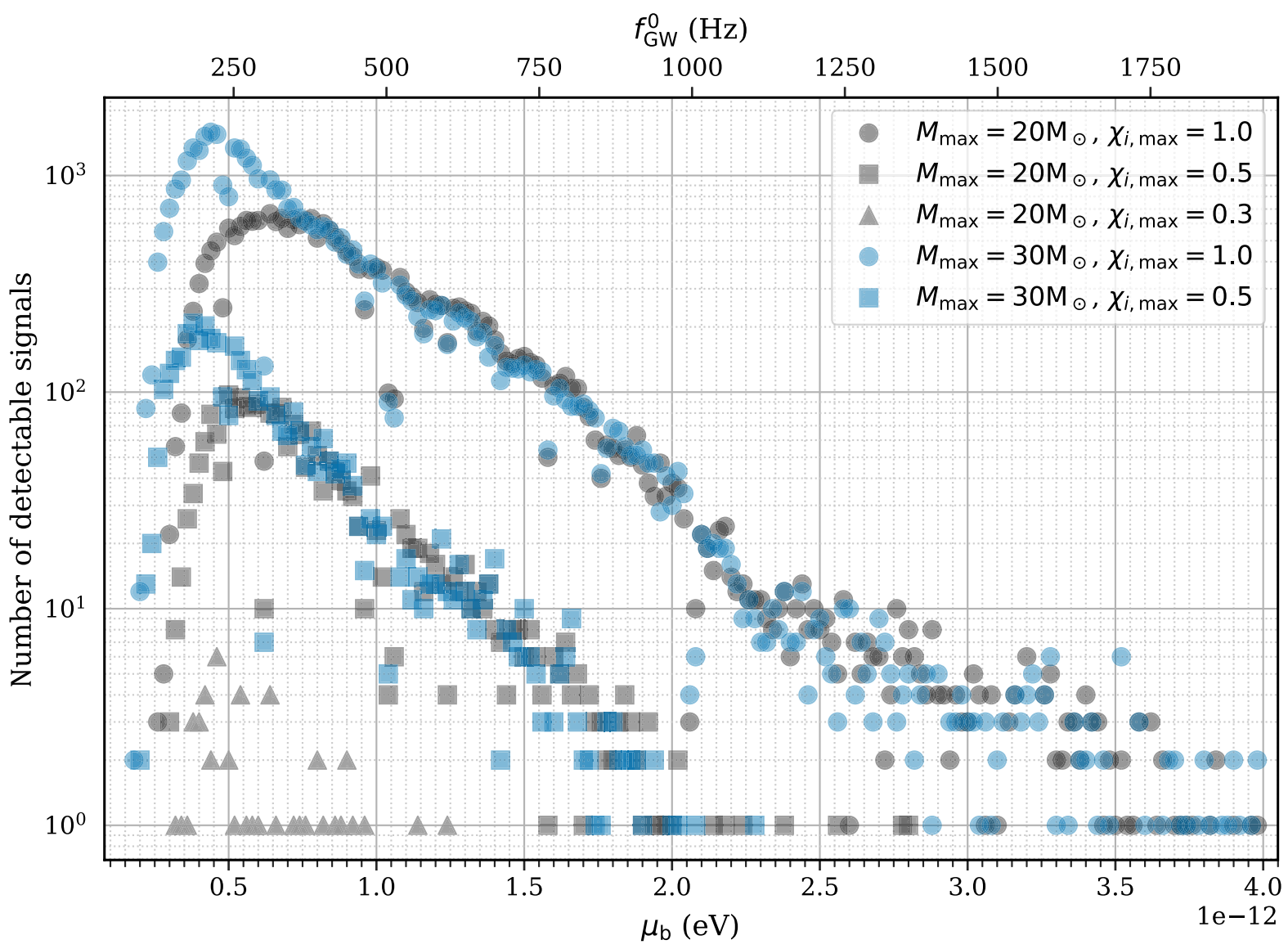

FIG. 18. Number of signals with amplitudes above the detectability level of recent all-sky searches for continuous gravitational waves [20-22], for bosons in the mass range $1 \times 10^{-13}$ to $4 \times 10^{-12} \mathrm{eV}$. The Falcon search [20,21] provides $95 \%$ confidence strict upper limits on continuous wave emission with near-zero frequency derivative between 20 and $600 \mathrm{~Hz}$. These upper limits are valid for any sky position and polarization of the source, even the ones with the most unfavorable coupling to the detectors. In contrast, the upper limits from the Freq Hough search [22] hold for 95\% of the entire population. For this reason, even at the same sensitivity, the Freq Hough upper limits are lower than the Falcon upper limits. We use the Falcon upper limits for bosons with $\mu_{\mathrm{b}}<1.2 \times 10^{-12} \mathrm{eV}$ and the Freq Hough upper limits for bosons with $\mu_{\mathrm{b}} \geq 1.2 \times 10^{-12} \mathrm{eV}$. We consider the standard (black circles) and heavy (blue circles) black hole populations, as well as the moderate (gray squares), heavy moderate (blue squares), and pessimistic (gray triangles) spin populations. The fluctuations are due to stationary lines and other artifacts in the detectors, which decrease the continuous wave search sensitivity in nearby frequency bins. 
and IV D, the signal strength grows with boson mass but the number of emitting systems decreases with increasing boson mass. The two competing effects combined with the sensitivity curve of the LIGO detectors (the noise is smallest at $\sim 170 \mathrm{~Hz}$ ) determine the boson mass $\mu_{\mathrm{b}}$ with the largest number of detectable signals.

For a given spin distribution, a heavier black hole population $\left(M_{\mathrm{BH}, \max }=30 \mathrm{M}_{\odot}\right)$ produces more signals for light bosons $\left(\mu_{\mathrm{b}}<8 \times 10^{-13} \mathrm{eV}\right)$ than does a lighter black hole population $\left(M_{\mathrm{BH} \text {, max }}=20 \mathrm{M}_{\odot}\right)$. For the heavy black hole population, the largest number of detectable signals occurs for $\mu_{\mathrm{b}}=4 \times 10^{-13} \mathrm{eV}$; for the lighter black hole population, the largest number corresponds to $\mu_{\mathrm{b}}=6 \times 10^{-13} \mathrm{eV}$.

In the case in which $M_{\mathrm{BH} \text {,max }}<20 \mathrm{M}_{\odot}$, fewer signals would be produced at the lightest boson masses. At a fixed value of $\alpha$, the strain is linearly proportional to black hole mass, $h_{0 \text {, peak }} \propto M_{\mathrm{BH}}$, so even decreasing the maximum black hole mass by a factor of 1.5 (already in tension with known Galactic black holes [98]) would shift the peak of the detectable signals to bosons a factor of 1.5 heavier.

For $\mu_{\mathrm{b}}>8 \times 10^{-13} \mathrm{eV}$, the two black hole populations produce the same number of detectable signals. For these heavier bosons, systems with heavier black holes form less frequently and have significantly shorter emission lifetimes and do not contribute to the event rates.

For a given black hole mass distribution, the number of detectable signals is most sensitive to the black hole spin distribution, through the superradiance condition of Eq. (4). We consider three different uniform spin distributions: a standard distribution with $\chi_{i} \in[0,1]$, a moderate distribution with $\chi_{i} \in[0,0.5]$, and a pessimistic distribution with $\chi_{i} \in[0,0.3]$.

The standard and moderate spin distributions produce detectable signals for boson masses $2 \times 10^{-13} \mathrm{eV} \lesssim \mu_{\mathrm{b}} \lesssim$ $2.5 \times 10^{-12} \mathrm{eV}$, although the number of detectable signals decreases by an order of magnitude from $\chi_{i, \max }=1$ to 0.5 , as fewer black holes have initial spins above the critical spin. For the pessimistic spin distribution, only a handful of signals are detectable, and only for boson masses $4 \times 10^{-13} \mathrm{eV} \lesssim \mu_{\mathrm{b}} \lesssim 1.3 \times 10^{-12} \mathrm{eV}$. The small signal number, in conjunction with there being no signals with $h_{0}>10^{-25}$ for $\chi_{i, \max }<0.3$ (Fig. 13), suggests that if all black holes are born with spins smaller than 0.3, no boson mass will produce a significant number of signals detectable by current continuous wave searches.

\section{B. Density of signals}

Since searches are designed under specific assumptions about signal properties, their performance may be degraded when the signals do not meet those assumptions. An important property of the target signal population is the number of detectable signals in a parameter space search cell. If the total signal is an incoherent superposition of many signals that cannot be resolved individually, then the total signal is a stochastic process that can best be detected with cross-correlation methods [99]. On the other hand, if the signals are sparse enough that they can be individually resolved, the matched filtering and semicoherent techniques that are commonly used to search for continuous wave signals should be applied [20,100-102]. Between these two extremes is a regime with some loud and resolvable signals on a background of weaker and unresolved ones, which may act like confusion noise.

We now want to understand what situation applies here. To address this question, we consider the Freq Hough [22] and Falcon searches [20,21].

We bin the signal frequency with the resolution of the first stage of the respective continuous wave search. We compute the ensemble signal for a standard black hole population and count the number of signals in each frequency bin with amplitude equal to or greater than the amplitude upper limit from the given continuous wave search, as a function of the signal frequency. For every bin, we also determine the mean and maximum signal amplitude over the ensemble signals with frequency in that bin and amplitude greater than the search upper limit amplitude. Figures 19 and 20 show these quantities for $\mu_{\mathrm{b}}=4 \times 10^{-13} \mathrm{eV}$, which is close to the boson mass yielding the highest number of detectable signals per bin. The maximum number of signals in a single Freq Hough bin is $\sim 10$, whereas for Falcon it is 2 . This difference is due mostly to the fact that the Freq Hough frequency bin is 14 times larger than the Falcon bin. We show the equivalent figures for a range of boson masses in Appendix E. As the number of signals scales linearly with the number of black holes, a smaller number of black holes could still yield multiple signals in a single bin for some combination of boson mass and maximum black hole mass. For instance, a population of $10^{7}$ black holes produces multiple signals in a single Freq Hough bin for boson masses of $3 \times 10^{-13} \mathrm{eV}$, assuming that $M_{\mathrm{BH}, \max }=30 \mathrm{M}_{\odot}$ (Fig. 35).

Although there are many Freq Hough signal-frequency bins that contain multiple signals, these signals are in principle still resolvable by the search because they come from different sky locations. However, due to large-scale parameter correlations of the detection statistics, sufficiently strong signals produce high detection statistic values even at template parameters far from the signal parameters. This is evident, for instance, in [103]; in spite of this being a search aimed at the Galactic Center, it still detects all LIGO hardware-injected fake signals at sky locations far from the Galactic Center. This means that when the ensemble signal comprises many loud signals in nearby frequency bins, even if each signal comes from a different sky location, it will contribute to the detection statistics at the templates of other signals, possibly giving rise to contamination/confusion noise. So the high concentration of detectable signals in many neighboring 

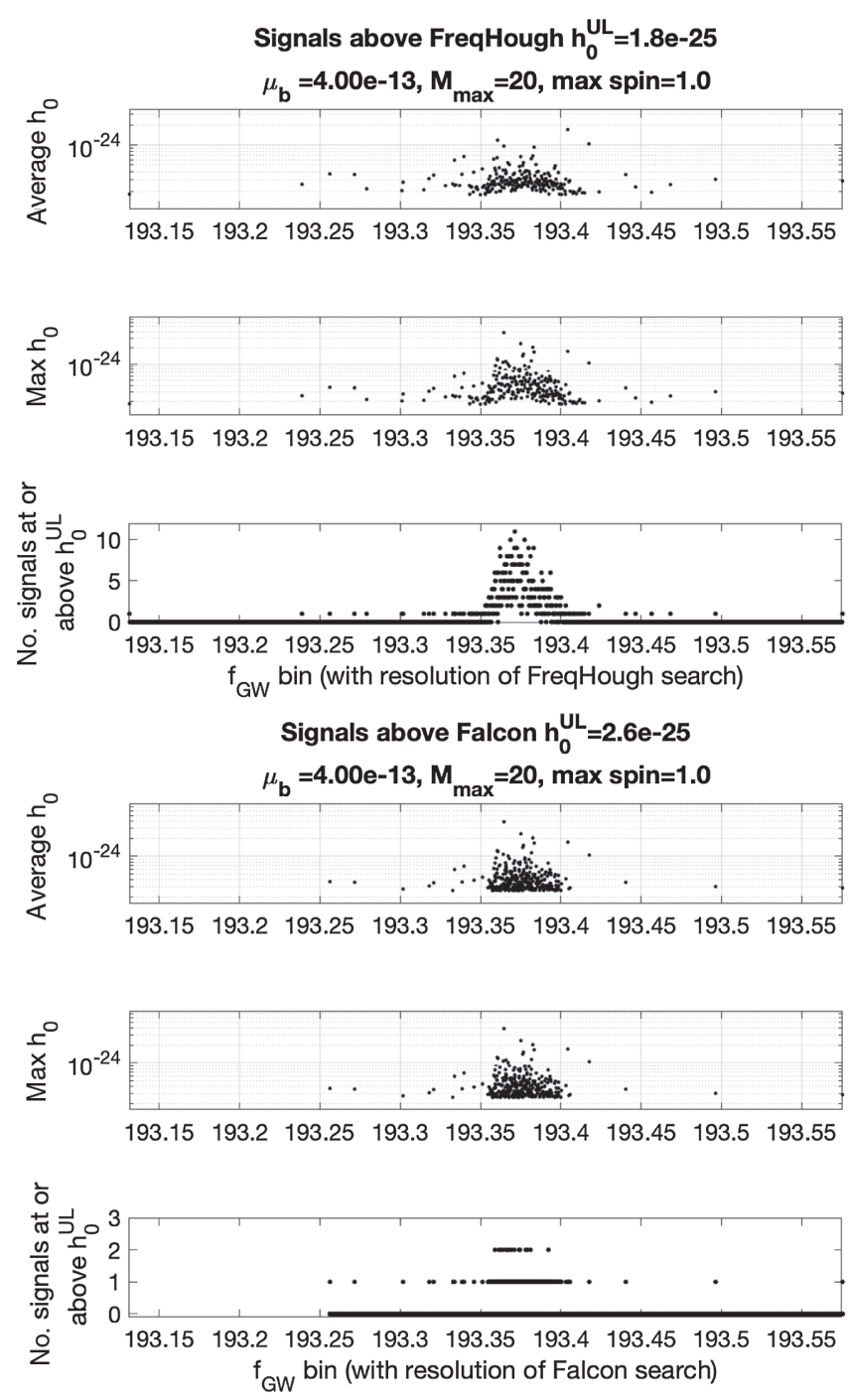

FIG. 19. The $x$ axes show the signal frequency binned with the resolution of the two searches: $2.4 \times 10^{-4} \mathrm{~Hz}$ for the Freq Hough search and $1.7 \times 10^{-5} \mathrm{~Hz}$ for the Falcon search. From the bottom panel going up, the $y$ axis shows the number of detectable signals in each bin, their maximum intrinsic amplitude, and their average amplitude. The main reason why there are fewer signals per frequency bin in the Falcon searches [20,21] than in the Freq Hough search [22] is that the frequency bin of Freq Hough is significantly larger (by a factor of 14) than the frequency bin of Falcon. The maximum initial spin of the black hole population is taken to be 1 .

frequency bins calls for caution while interpreting results from continuous wave searches for rare signals as boson annihilation signals.

A large signal density has consequences because the complex all-sky continuous wave search pipelines [20-23, 104] comprise several follow-up stages of increasing coherence lengths, with vetoes and thresholds designed to reject noise fluctuations and coherent disturbances while preserving signal candidates. The procedures are carefully calibrated with extensive Monte Carlo simulations on fake
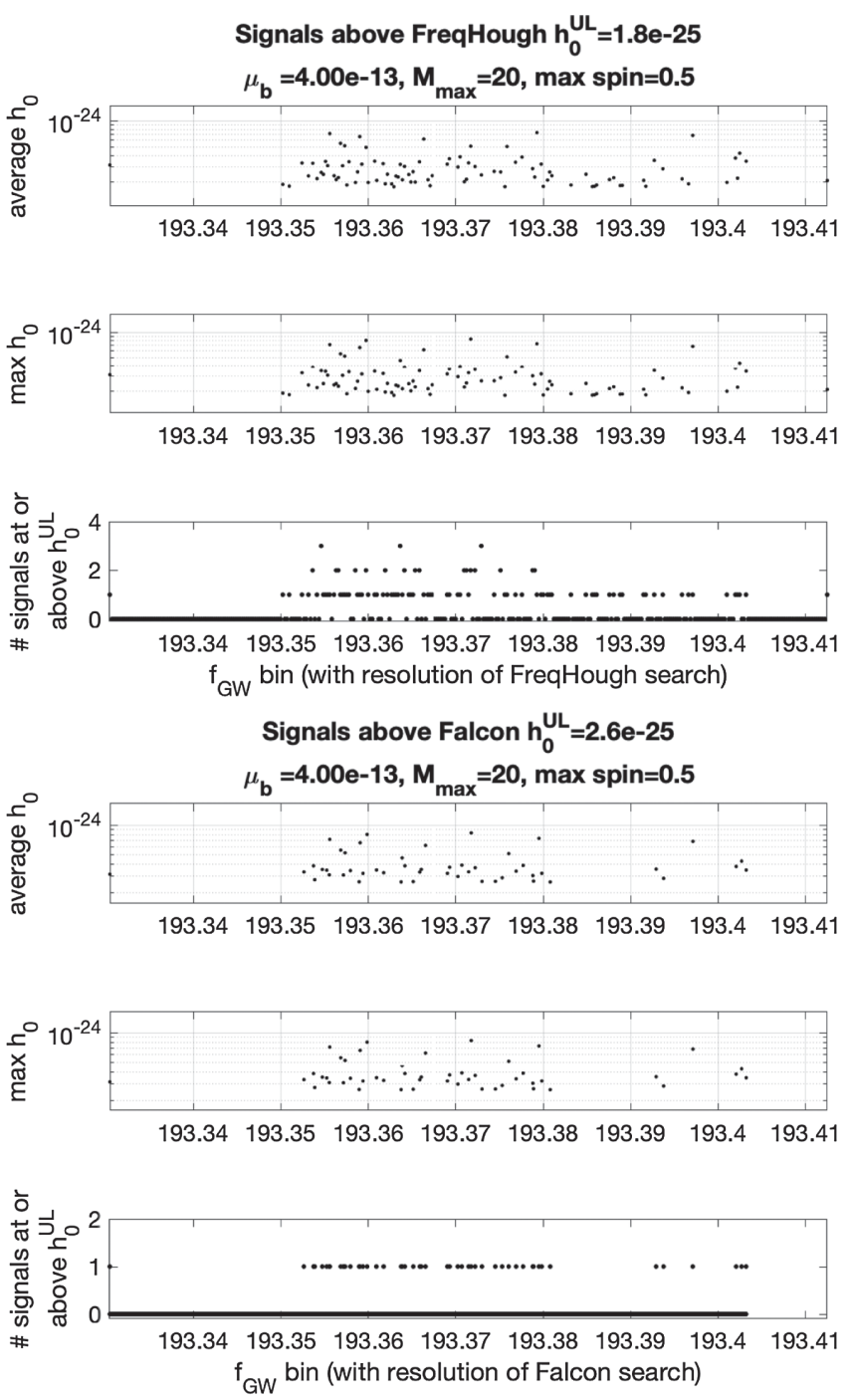

FIG. 20. Same plots as those in Fig. 19 but with the assumption that the maximum initial spin of the black hole population is 0.5 .

isolated signals added to real noise. It is unlikely that settings that have been tuned to isolated signals would be equally effective for the ensemble signals explored in this study, especially in regions of high signal density. We expect that the detection efficiency of searches for which the density and number of detectable signals per bin is larger will be impacted more severely; so, for example, the impact will be greater for the Freq Hough search [22] than the Falcon search [20,21].

We note that in Fig. 19, the frequencies where the signals are the strongest are also the frequencies with the highest density of signals. This is not the case in general. As discussed in Sec. IV B, for a given boson mass, systems with heavier black holes produce signals with lower frequencies and larger strains; at the same time, heavier black holes are rarer and have larger minimum spins required for cloud formation. This causes the typical "peaked" shape (Fig. 9) of the ensemble signal so that 


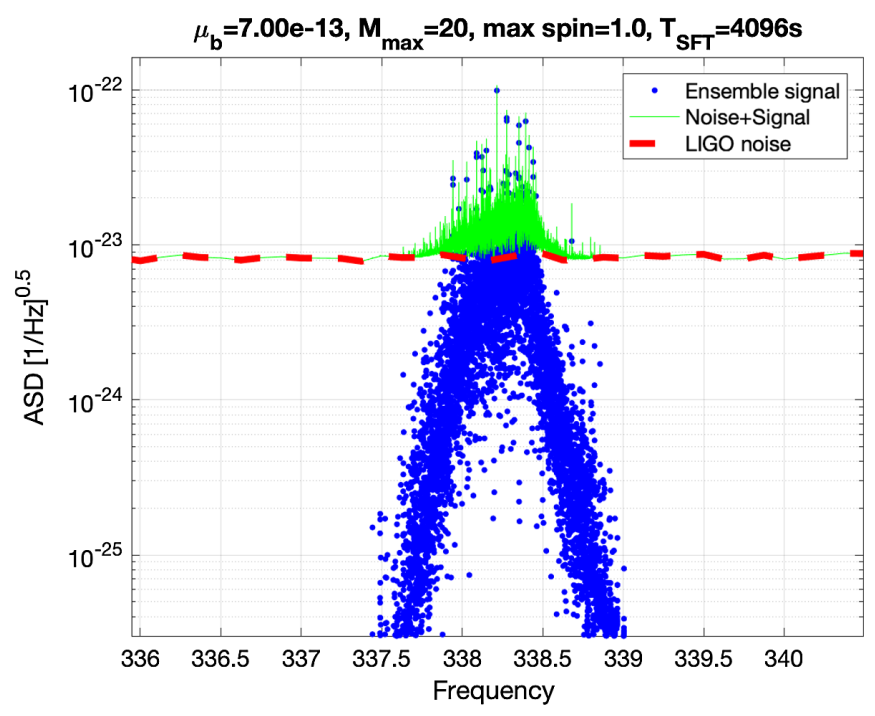

FIG. 21. The amplitude spectral density of the LIGO O2 data alone (dashed red line) and of an ensemble signal (top green points) assuming that $\mu_{\mathrm{b}}=7 \times 10^{-13} \mathrm{eV}$ and a Galactic black hole population with maximum mass $20 \mathrm{M}_{\odot}$ and maximum initial spin of 1 . The time baseline assumed is $4096 \mathrm{~s}$, as used by the Freq Hough search [22].

the loudest signals tend to be located at or below the frequencies with the densest clustering of signals, as can be seen in Appendix E.

A high density of loud signals in a broad enough frequency range could also have a more subtle negative impact on the ability of standard continuous wave searches to detect signals from the ensemble. The reason is that the average noise level data is "normalized out" of the detection statistic, but if this is not done carefully, there is a danger of reducing the signal. Most continuous wave searches (and all of the ones that we refer to in this paper) whiten the data at an early point in the analysis by dividing the fast Fourier transform of the data taken over some short time baseline (SFT) with the amplitude spectral density estimated from the data itself. The noise level is estimated through an average of the noise power over tens of SFT bins. Under the assumption that the data is noise dominated, with at most a single detectable signal concentrated in a SFT bin or two, the "average" over tens of bins is insensitive to the signal. For instance, Freq Hough [22] searches use an autoregressive average on the power spectral density over $\lesssim 0.02 \mathrm{~Hz} \quad[105,106]$ and the Einstein@Home searches [23,104] use running medians over $\lesssim 0.06 \mathrm{~Hz}$ frequency bins.

If the ensemble signal is a collection of many signals loud enough to increase the apparent noise floor in a broad enough band in the input data (the SFTs), then the noise estimate will be sensitive to this increase, the search normalization procedure will down weight the data containing the signals, and this might decrease the detection statistic associated with some of the ensemble signals.
In this case, the upper limits derived for isolated signals should be used with caution when reinterpreted to apply to ensemble signals. In Fig. 21, we plot the amplitude spectral density (ASD) expected for the ensemble signal corresponding to $\mu_{\mathrm{b}}=7 \times 10^{-13} \mathrm{eV}$. There clearly is additional power due to the ensemble signal extending over $\gtrsim 1 \mathrm{~Hz}$. This excess would also appear in the noise estimate and, for the reason explained above, could change the detection efficiency curve as a function of signal amplitude with respect to the isolated signal case. More plots like that in Fig. 21 are given in Appendix F for different combinations of boson mass, maximum black hole mass, and maximum initial black hole spin. For $3 \leq \mu_{\mathrm{b}} \leq 7 \times 10^{-13} \mathrm{eV}$, an increased ASD on the SFT timescale is visible, except for ensembles with initial maximum black hole spin 0.3 or less and the maximum black hole mass of $20 \mathrm{M}_{\odot}$.

\section{EXISTING LITERATURE AND RESULTS}

Several works have made projections of the detectability of the boson annihilation signal at Advanced LIGO and Virgo [12-14]. These studies predict tens to thousands of signals detectable with Advanced LIGO/Virgo continuous wave searches, with a range of assumptions about black hole mass and spin distributions in combination with often only idealized search concepts. Other works have searched the gravitational-wave data for boson annihilation signals leveraging existing search pipelines and/or results from other astrophysical searches.

All-sky O2 continuous wave survey nondetection results of [22] are used to exclude bosons with $1.1 \times 10^{-13} \mathrm{eV}<$ $\mu_{\mathrm{b}}<4 \times 10^{-13} \mathrm{eV}$ assuming that all black holes are formed with spin 0.998, or $1.2 \times 10^{-13} \mathrm{eV}<\mu_{\mathrm{b}}<1.8 \times$ $10^{-13} \mathrm{eV}$ assuming that all black holes are formed with spin 0.6 , and a range of $3 \mathrm{M}_{\odot}-100 \mathrm{M}_{\odot}$ black hole masses in both cases. We find that the number of signals below $\sim 3 \times$ $10^{-13} \mathrm{eV}$ falls off very rapidly for both $M_{\max , \mathrm{BH}}=30 \mathrm{M}_{\odot}$ and $M_{\max , \mathrm{BH}}=20 \mathrm{M}_{\odot}$ (Fig. 18), indicating that the excluded masses of [22] rely on a speculative population of heavy black holes. Our analysis of the ensemble signal shows that any bound derived from a single spin or distance assumption can result in misleading conclusions. Furthermore, the ensemble signal from bosons with mass between $3 \times 10^{-13}$ and $4 \times 10^{-13} \mathrm{eV}$ would likely be detected with a lower efficiency than that of an isolated signal, and hence nondetections from a standard continuous wave search cannot automatically be translated into exclusions for boson signals at the same frequency at the same $h_{0}$ level.

A cross-correlation search technique is considered in [19]: the boson annihilation ensemble signal from outside the Milky Way is treated as a stochastic background [15]. Based on null results, Tsukada et al. [19] excluded the range $2.0 \times 10^{-13}-3.8 \times 10^{-13} \mathrm{eV}$ under an "optimistic" black hole spin distribution (defined as unity maximum 
dimensionless spin and letting the minimum vary) but not under a "pessimistic" one (zero minimum spin while letting the maximum vary). The range of black hole masses considered is $3 \mathrm{M}_{\odot}-100 \mathrm{M}_{\odot}$. The amplitude spectral density estimates based on our ensemble signal suggest that their null result could exclude Galactic signals from some boson masses even for maximum initial black hole spins of 0.5 , as long as the black hole masses go at least up to $30 \mathrm{M}_{\odot}$.

A way to bypass the uncertainties in the system's parameters is to consider a known black hole, for example, the remnant of a merger of two compact objects [13]. For the LIGO black holes, the formation time is precisely known, and the spin and mass are measured with good precision but the remnant is too far away to yield a signal detectable by the current interferometers [13,107]. Turning to galactic sources, Sun et al. [108] searched for continuous wave emission from Cygnus X-1, a well-known system: a black hole of $\sim 15 \mathrm{M}_{\odot}, 5 \times 10^{6}$ years old at a distance of $1.86 \mathrm{kpc}$. The absence of a detection disfavors the existence of bosons in the range $5.8 \times 10^{-13}-8.6 \times 10^{-13} \mathrm{eV}$. This result, however, assumes a near-extremal initial black hole spin of 0.99 and implies a low current spin, $\chi \in[0.25,0.36]$, which is in tension with continuum and reflection measurements of the spin, $\chi \gtrsim 0.95[109,110]$.

\section{CONCLUSIONS}

In this work, we simulate the ensemble annihilation signal from a population of isolated Galactic black holes and predict the signal detectability using recent all-sky continuous wave searches. We study the dependence of the ensemble signal on black hole population properties. We show how population assumptions, in particular, on black hole mass and spin distributions, can strongly affect the detectability of the ensemble signal. We propose that the results of future boson annihilation signal searches be interpreted using the ensemble-signal paradigm that we present here. We have made the ensemble-signal population parameters used in this work publicly available to facilitate future studies [96].

Our analysis is the first study to characterize the unique shape of the ensemble signal-constructed from a range of distributions of the underlying parametersand suggests a clear way to distinguish between these signals and the isolated signals produced by rotating neutron stars.

We choose a population of $10^{8}$ black holes as a benchmark. For a population with the same mass, age, and initial spin distribution but a different number of black holes, simple scaling arguments can be applied to our results in order to predict the results for that population. A study of the impact of different black hole population parameters on the boson mass constraints is very interesting but outside the scope of this paper. On the other hand, the ensemble signal approach that we propose here is exactly meant to enable this type of study.
We have considered the signals from five different black hole populations. We use two mass distributions: the standard black hole mass distribution (maximum black hole mass $M_{\mathrm{BH} \text {,max }}=20 \mathrm{M}_{\odot}$ ) covers the range of masses for known Galactic black holes [98] and is therefore conservative, while the heavy black hole population $\left(M_{\mathrm{BH}, \max }=30 \mathrm{M}_{\odot}\right)$ allows us to test how the properties of the ensemble signal depend on the heaviest black holes. We also consider three different initial spin distributions: the optimistic spin distribution of $\chi_{i} \in[0,1]$, the moderate spin distribution of $\chi_{i} \in[0,0.5]$, and the pessimistic spin distribution of $\chi_{i} \in[0,0.3]$. For all populations, we assume a total of $10^{8}$ Galactic black holes. The predicted number typically varies between $10^{7}$ and $10^{8}$ and is highly uncertain, as there are no direct observations; the number of signals scales proportionally to the black hole number.

Assuming there are $10^{8}$ isolated black holes in the Galaxy with $M_{\mathrm{BH}} \in\left[5 \mathrm{M}_{\odot}, 30 \mathrm{M}_{\odot}\right]$ and initial spin $\chi_{i} \in[0,1]$, bosons with masses $\sim 2.5-17 \times 10^{-13} \mathrm{eV}$ produce 100 or more signals with amplitude above the upper limits in the $\mathrm{O} 1 / \mathrm{O} 2$ continuous wave searches, and masses $\sim 4.5 \times 10^{-13} \mathrm{eV}$ produce over 1000 signals above those upper limits $[20,21,47,97]$. For a lighter population of black holes $\left(M_{\mathrm{BH}} \in\left[5 \mathrm{M}_{\odot}, 20 \mathrm{M}_{\odot}\right]\right)$, the boson mass range yielding signal amplitudes above the existing upper limits is not significantly different than the range for the heavier population - the lowest boson mass yielding 100 or more signals increases to $\sim 3.5 \times 10^{-13} \mathrm{eV}$-and the highest number of signals is a factor of $\sim 2$ lower.

If we take the maximum black hole spin at birth to be 0.5 rather than 1.0, keeping $M_{\mathrm{BH} \text {,max }}=20 \mathrm{M}_{\odot}$, we find that the number of detectable signals drops by an order of magnitude. This suggests that black holes with $\chi_{i}>0.5$ produce $90 \%$ of the "detectable" signals, so even if only $10 \%$ of black holes are born with $\chi_{i}>0.5$, we predict tens to hundreds of detectable signals for $3 \times 10^{-13} \mathrm{eV} \lesssim \mu_{\mathrm{b}} \lesssim 17 \times 10^{-13} \mathrm{eV}$. The number of detectable signals drops by at least another order of magnitude if the maximum spin at birth is 0.3 , and black holes with $\chi_{i}$ between 0.3 and 0.5 produce $\sim 10 \%$ of the detectable signals. If instead all black holes are born with spins $\lesssim 0.3$, we expect ten or fewer signals across the entire frequency range. Given other uncertainties such as the overall number of Galactic black holes, in this regime even a single detectable signal is not assured.

We caution that for boson masses in the range $\sim 3 \times 10^{-13}-10 \times 10^{-13} \mathrm{eV}$, the sensitivity of standard continuous wave search methods that have been carefully tuned for the regime of quiet and very sparse signals, should be characterized again on a dense ensemble of loud signals. The interplay between the potential down weighting of the signal from the normalization of the data and the overlap in parameter space of the detection statistic from different signals in the ensemble and how these elements factor in a multistage follow-up process needs to be investigated. For boson masses between $4 \times 10^{-13}-6 \times 10^{-13} \mathrm{eV}$, even for 
moderately rotating black hole populations, the GW ensemble signal is very prominent, which is a dramatically different regime than the one assumed by standard continuous wave searches.

While the detection of a single particularly loud signal from a nearby black hole could serendipitously occur even with a highly "mismatched" search, ultimately the identification of a boson annihilation signature-as opposed to a continuous wave signal from a compact rotating objectlies in the identification of the ensemble signal. Searches for gravitational-wave signals from boson clouds around black holes are only starting to be explored. This work lays the foundation for this type of detectability study.

\section{ACKNOWLEDGMENTS}

The authors thank Richard Brito for fruitful discussions, and for providing the numerical results and uncertainties of the annihilation power. We thank Bruce Allen, Pia Astone, Banafsheh Beheshtipour, Alessandra Buonanno, Horng Sheng Chia, Vladimir Dergachev, Badri Krishnan, Andrew MacFadyen, and Cole Miller for useful discussions. M. B. is supported by the James Arthur Postdoctoral Fellowship. D. T. is supported by the Advanced Leading Graduate Course for Photon Science at the University of Tokyo, and by JSPS KAKENHI Grant No. JP19J21578. N.K. is supported by the Hakubi project at Kyoto University.

\section{APPENDIX A: GENERAL FORMULAS FOR SCALAR SUPERRADIANCE}

In this appendix we provide the more general forms for the equations that are used in the text. All analytic formulas are valid for $\alpha / \ell \ll 1$. We find that the potentially detectable signals in this work arise from systems with $0.03 \lesssim \alpha \lesssim 0.2$. We also comment on the assumptions and uncertainties that enter into the signal calculations.

\section{Signal frequency}

As the signal comes from the annihilation of two bosons, the frequency of the emitted gravitational wave $f_{\mathrm{GW}}$ is given by twice the boson energy $\omega_{R}$ :

$$
f_{\mathrm{GW}}^{n \ell m}=\frac{\omega_{\mathrm{GW}}}{h}=\frac{2 \omega_{R}}{h} .
$$

The energy of the boson is given by

$$
\omega_{R}^{n \ell m}=\mu_{\mathrm{b}}-\Delta \omega_{R, \mathrm{BH}}^{n \ell m}-\Delta \omega_{R, \mathrm{cloud}}^{n \ell m},
$$

where $\Delta \omega_{R, \mathrm{BH}}^{n \ell m}$ is the gravitational potential energy and $\Delta \omega_{R, \text { cloud }}^{n \ell m}$ the gravitational self-energy of the cloud. The potential energy is given by [43]

$$
\begin{aligned}
\Delta \omega_{R, \mathrm{BH}}^{n \ell m} \simeq & \mu_{\mathrm{b}}\left(\frac{\alpha^{2}}{2(\ell+n+1)^{2}}+\frac{\alpha^{4}}{8(\ell+n+1)^{4}}\right. \\
& -\frac{2 \ell-3(\ell+n+1)+1}{(\ell+n+1)^{4}(\ell+1 / 2)} \alpha^{4} \\
& \left.-\frac{2 \chi_{i} m \alpha^{5}}{(\ell+n+1)^{3} \ell(\ell+1 / 2)(\ell+1)}\right),
\end{aligned}
$$

and the uncertainty on the analytic calculation compared to the numerical result was shown to be $\lesssim 1 \%$ for $\alpha \lesssim 0.3$, becoming an excellent approximation for $\alpha \lesssim 0.2$ [43].

The self-energy of the cloud depends on the mass of the cloud in the nonrelativistic limit as $[39,107]$

$$
\Delta \omega_{R, \text { cloud }}^{n \ell m} \approx \mu_{\mathrm{b}}\left(0.2 \alpha^{2} \frac{M_{\text {cloud }}}{M_{\mathrm{BH}}}\right) .
$$

The analogous calculation for a vector in the state $n=0, j=1, \ell=0, m=1$ varies by up to $50 \%$ for $\alpha<$ 0.3 depending on whether the relativistic corrections of the wave function are included [111]. We expect the uncertainty on our calculation to be smaller, as the vector states (with $\ell=0$ ) are localized closer to the black hole than the scalar states (with $\ell=1$ ) and therefore are more affected by relativistic effects.

For $0.01 \lesssim \alpha \lesssim 0.3$, we find that the self-energy contribution is much smaller than the black hole gravitational potential contribution, $10^{-6} \lesssim \Delta \omega_{R, \text { cloud }}^{n \ell m} / \Delta \omega_{R, \mathrm{BH}}^{n \ell m} \lesssim 10^{-3}$. This is less than the error on the analytic estimate of Eq. (A3); nevertheless, as this quantity is time dependent, it determines the frequency drift of the signal; see Appendix A 5.

\section{Superradiance rate}

The instability rate of the boson is $[18,37]$

$$
\begin{aligned}
\omega_{s r}^{n \ell m} \approx & 2 \mu_{b} C_{n \ell m}(\alpha, \chi) \alpha^{4 \ell+4} \\
& \times\left(1+\sqrt{1-\chi^{2}}\right)\left(\frac{m \chi}{2\left(1+\sqrt{1-\chi^{2}}\right)}-\alpha_{\omega}\right),
\end{aligned}
$$

where $\chi$ is the dimensionless black hole spin and $C_{n \ell m}(\alpha, \chi) \ll 1$ is a numerical coefficient which is a function of the bound state quantum numbers as well as $(\alpha, \chi)$,

$$
\begin{aligned}
C_{n \ell m}(\alpha, \chi)= & \frac{2^{4 \ell+2}(2 \ell+n+1) !}{n !(\ell+n+1)^{2 \ell+4}}\left(\frac{\ell !}{(2 \ell) !(2 \ell+1) !}\right)^{2} \\
& \times \prod_{j=1}^{\ell}\left(4\left(\sqrt{1-\chi^{2}}+1\right)^{2}\left(\frac{m \chi}{2\left(\sqrt{1-\chi^{2}}+1\right)}-\alpha_{\omega}\right)^{2}\right. \\
& \left.+j^{2}\left(1-\chi^{2}\right)\right) .
\end{aligned}
$$


We define $\alpha_{\omega} \equiv \alpha \omega_{R} / \mu$, i.e., the $\alpha$ corrected for the potential energy contribution to the boson energy. The fastest-growing level for a light scalar, the $n=0, \ell=m=1$ level, has $\omega_{s r}^{011} \simeq \frac{1}{24} \chi \alpha^{8} \mu_{\mathrm{b}}$.

The rate for $\ell=1$ is larger than that for $\ell=2$ by a factor of $\sim C_{011} / C_{022} \alpha^{-4} \sim 10^{3} \alpha^{-4}$.

The instability $e$-folding timescale is then given by

$$
\tau_{\mathrm{inst}}^{n \ell m}=\frac{\hbar}{\omega_{s r}^{n \ell m}},
$$

with $\ln (N)=\ln \left(M_{\mathrm{BH}} c^{2} / \mu_{\mathrm{b}}\right) \sim 180$ instability timescales required to fully saturate the cloud growth. The analytical form is accurate to within a factor of 2 for $\alpha \lesssim 0.25$ and within $50 \%$ for $\alpha \lesssim 0.2 .^{3}$

\section{Final cloud mass}

Superradiance will start if the superradiance condition is satisfied, i.e., the initial spin of the black hole is above the critical spin,

$$
\chi_{c}^{n l m}=\frac{4 M_{\mathrm{BH}} \omega_{R} m}{m^{2}+4 M_{\mathrm{BH}}^{2} \omega_{R}^{2}} \approx \frac{4 \alpha m}{m^{2}+4 \alpha^{2}} .
$$

As the black hole loses mass and angular momentum, the superradiance condition and thus the final spin are affected. The equations governing the evolution are

$$
\begin{gathered}
\dot{N}(t)=\omega_{s r}^{n \ell m}(t) N(t), \\
\dot{M}(t)=-\omega_{R}^{n \ell m}(t) \dot{N}(t), \\
\dot{J}(t)=-\dot{N}(t),
\end{gathered}
$$

where $N(t)$ is the number of particles in the cloud, $M(t)$ is the black hole mass, and $J(t)=G M(t)^{2} \chi(t)$ is the angular momentum of the black hole. The superradiance rate and the energy of the boson depend on time through their implicit dependence on $\alpha(t)=G \mu M(t)$ and $\chi(t)=J(t) /\left(G M(t)^{2}\right)$.

The process saturates when

$$
\chi_{f}=\frac{4 M_{\mathrm{f}} \omega_{R, f}}{1+4 M_{\mathrm{f}}^{2} \omega_{R, f}^{2}} .
$$

By conservation of angular momentum, the number of particles in the cloud is given by the angular momentum lost by the black hole, $N_{f}=J_{i}-J_{f}$, with each particle carrying one unit of angular momentum (for $\ell=m=1$ ). The mass of the cloud is given by the number of particles times their energies,

\footnotetext{
${ }^{3}$ We thank Horng Sheng Chia for providing the numerical rate comparison.
}

$$
M_{\text {cloud }}=\omega_{R} N_{f}=\omega_{R}\left(\chi_{i} G M_{i}^{2}-\chi_{f} G M_{f}^{2}\right) .
$$

In the limit $\alpha\left(\chi_{i}-\chi_{c}\right) \ll 1$, this reduces to

$$
M_{\text {cloud }}=\alpha\left(\chi_{i}-\chi_{c}\right) M_{i},
$$

which is accurate to $1 \%$ for $\alpha<0.03$ and underestimates the cloud mass by $\sim 50 \%$ for $\alpha \sim 0.2, \chi \sim 1$. We use the numerical evolution of $\alpha$ and $\chi$ to establish the final cloud mass.

\section{Gravitational-wave signal}

The power emitted in gravitational waves for $\alpha \ll 1$ has been computed analytically in the Schwarzschild background and gives [44]

$$
P_{\mathrm{GW}} \approx 0.025 \frac{c^{5}}{G} \alpha^{14} \frac{M_{\mathrm{cloud}}^{2}}{M_{\mathrm{BH}}^{2}} .
$$

At larger $\alpha>0.1$, we use the power calculated numerically in [14],

$P(\alpha)=\frac{1}{2 \pi} \frac{c^{5}}{G} \frac{M_{\text {cloud }}^{2}}{M_{\mathrm{BH}}^{2}}\left(\frac{\hbar c^{3}}{\omega_{\mathrm{GW}}^{n \ell m} G M_{\mathrm{BH}}}\right)^{2} \mathcal{A}_{\tilde{e} \tilde{m}}^{2}\left(\alpha, \chi_{i}\right)$,

where $\tilde{\ell}, \tilde{m}$ are spherical harmonics modes of the emitted gravitational radiation and $\mathcal{A}_{\tilde{\ell} \tilde{m}}$ is a dimensionless function that contains information about the fraction of energy deposited in the $\tilde{l}=\tilde{m}=2$ mode. The uncertainty in $\mathcal{A}$ ranges between $\sim 5 \%$ at intermediate $\alpha$ up to $\sim 15 \%$ at $\alpha<0.1$ and/or large spin. ${ }^{4}$ We use a polynomial interpolation between the low- $\alpha$ (Eq. (12) and large- $\alpha$ [Eq. (A16)] regime. We show the normalized power for the full range of $\alpha$ in Fig. 22.

For $\alpha \gtrsim 0.3$, the power emitted in modes with $\tilde{\ell}, \tilde{m}>2$ becomes dominant [45] and defines the depletion rate of the cloud. Since we have only considered the $\tilde{\ell}=\tilde{m}=2$ mode in our power calculations, the gravitational-wave emission timescales $\tau_{\mathrm{gw}}$ calculated here are an overestimate. However, this does not change our conclusions with respect to the signal detectability, as only systems with $\alpha \lesssim 0.2$ are detectable using current searches.

The maximal peak strain is related to the power emitted by the axion cloud as

$$
h_{0, \text { peak }}(\alpha)=\left(\frac{10 G \hbar^{2} P(\alpha)}{c^{3} \omega_{\mathrm{GW}}^{2} r^{2}}\right)^{1 / 2}
$$

where the two gravitational-wave polarizations are given by

\footnotetext{
${ }^{4}$ We thank Richard Brito for discussions on the details of the calculation and uncertainties.
} 


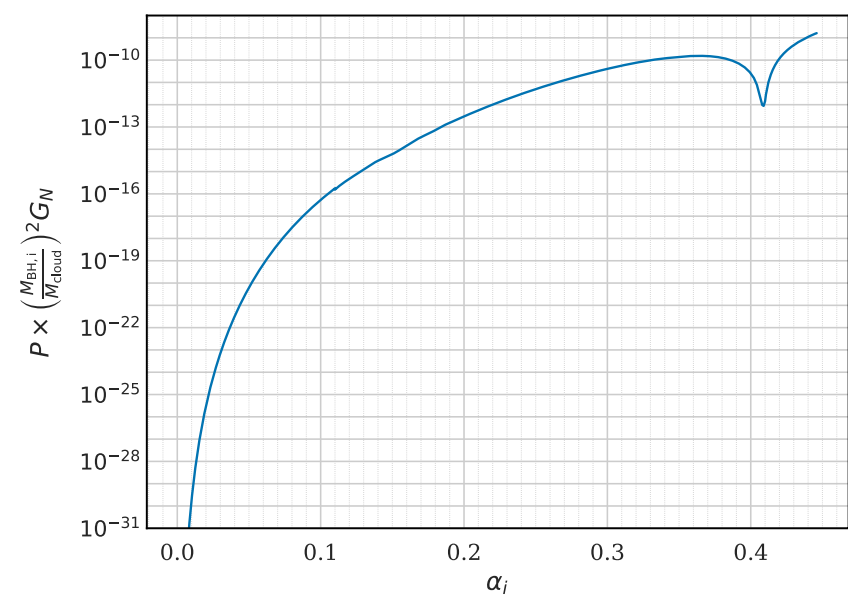

FIG. 22. The power emitted in the $\tilde{l}=\tilde{m}=2$ spherical harmonics mode of the gravitational radiation, normalized by $\left(M_{\mathrm{BH}, \mathrm{i}} / M_{\text {cloud }}\right) G_{N}$ to be a dimensionless quantity. The initial black hole spin assumed here is $\chi_{i}=0.99$; the curves for other values of $\chi_{i}$ are very similar and primarily differ in their maximum value of $\alpha_{i}$. The power for $\alpha_{i}>0.1$ is calculated numerically [Eq. (A16)] and is suppressed for some high spins, resulting in a "dip" near $\alpha \sim 0.4$. This directly affects the shape of $h_{0}$ and $\tau_{\mathrm{gw}}$.

$$
\begin{aligned}
& h_{+}(t)=\frac{1}{2} h_{0}\left(1+\cos ^{2} \imath\right) \cos \Phi(t), \\
& h_{\times}(t)=h_{0}(\cos \imath) \sin \Phi(t) .
\end{aligned}
$$

As the power is emitted in the vicinity of a Kerr black hole, the angular dependence is not exactly as defined in Eq. (A18) but is instead specified by spin-weighted spheroidal harmonics, which depend on $\alpha$ and the black hole spin $\chi[14,112]$. However, for $\alpha, \chi_{f}$ corresponding to $\alpha \lesssim 0.3(0.2)$, the standard quadrupolar emission is an excellent approximation to within $5 \%(3 \%)$ or better in $h_{\times}$and to within $10 \%(5 \%)$ or better in $h_{+}$. Given that the exact angular power calculation is computationally intensive and the continuous wave pipelines are optimized for strain angular dependence according to Eq. (A18), we neglect the extra effect of spin in our analysis. We also focus only of the $\tilde{\ell}=\tilde{m}=2$ gravitational-wave mode, which dominates the total power for $\chi \sim \chi_{c}$ and $\alpha \lesssim$ 0.35 [45].

We consider only the gravitational-wave emission from the $n=0, \ell=m=1$ cloud; this bound state produces the largest strain: using the values of [45], the strain of the first level is larger than that of the second by $h_{0}{ }^{011} / h_{0}{ }^{022} \sim 90 \alpha^{-2}$.

The time evolution of the signal as the cloud depletes through GW radiation is also related to the power emitted and is given by

$$
\tau_{\mathrm{GW}}(\alpha)=\frac{M_{\text {cloud }} c^{2}}{P(\alpha)}
$$

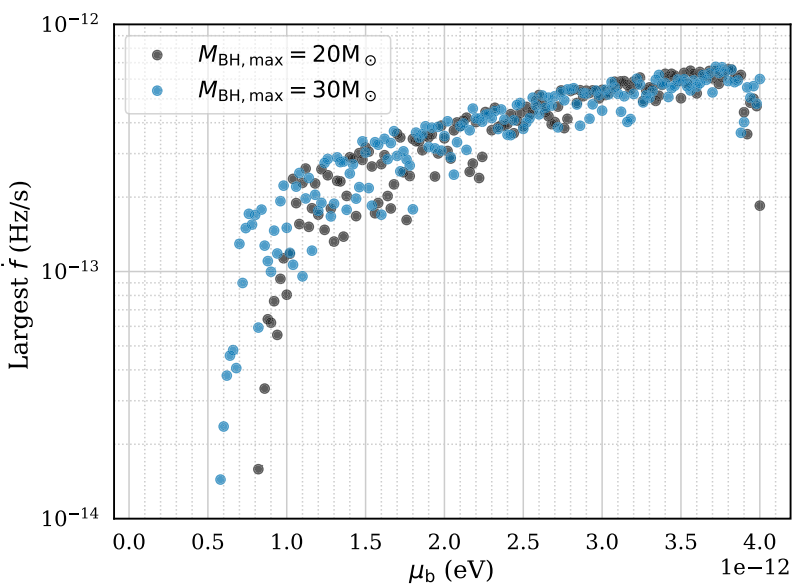

FIG. 23. The spin-up $\dot{f}$ due to the decreasing binding energy of the cloud depends on $\mu_{\mathrm{b}}$ and $M_{\mathrm{BH}}$ [Eq. (16)]. For both the standard and heavy black hole populations, the largest value of $\dot{f}$ is $6 \times 10^{-13} \mathrm{~Hz} / \mathrm{s}$.

The calculations of the power have been performed in the point particle approximation, i.e., the backreaction of the cloud on the metric is neglected. During the process of superradiance, the black hole spins down and loses mass to the cloud; thus, the emission is taking place approximately in a background defined by the final black hole mass and we use the final, smaller value of $\alpha$ to evaluate the strain and timescale expressions. The presence of the cloud may be viewed as an additional contribution to the mass in the Kerr metric, in which case the initial value of $\alpha$ could be used as an approximation [111]. Our expression is more conservative (as the final $\alpha$ is smaller and thus the power is reduced) and is correct in the limit when the cloud has a mass much smaller than that of the black hole, as is the case for many of our old, long-lasting signals. The difference between using the initial and final $\alpha$ gives approximately a $50 \%$ change in the total power, which is a conservative estimate of the overall uncertainty in the rate.

\section{Frequency drift}

As the cloud annihilates to gravitational waves, its gravitational potential energy decreases, leading to a positive frequency drift,

$$
\begin{aligned}
\dot{f}_{\mathrm{GW}}(t) \approx & 5 \times 10^{-15} \mathrm{~Hz} / \mathrm{s} \\
& \times\left(\frac{\alpha}{0.1}\right)^{19}\left(\frac{10 \mathrm{M}_{\odot}}{M}\right)^{2}\left(\frac{\chi_{i}-\chi_{c}}{0.5}\right)^{2}\left(\frac{M_{\text {cloud }}(t)}{M_{\text {cloud }}^{\max }}\right)^{2} .
\end{aligned}
$$

There is a larger, negative frequency drift at early times as the cloud is growing, but we neglect this in our analysis as the strain is small at these times.

In Fig. 23, we check to see that the $\dot{f}$ caused by the cloud's decreasing mass is still smaller than can be resolved by the 


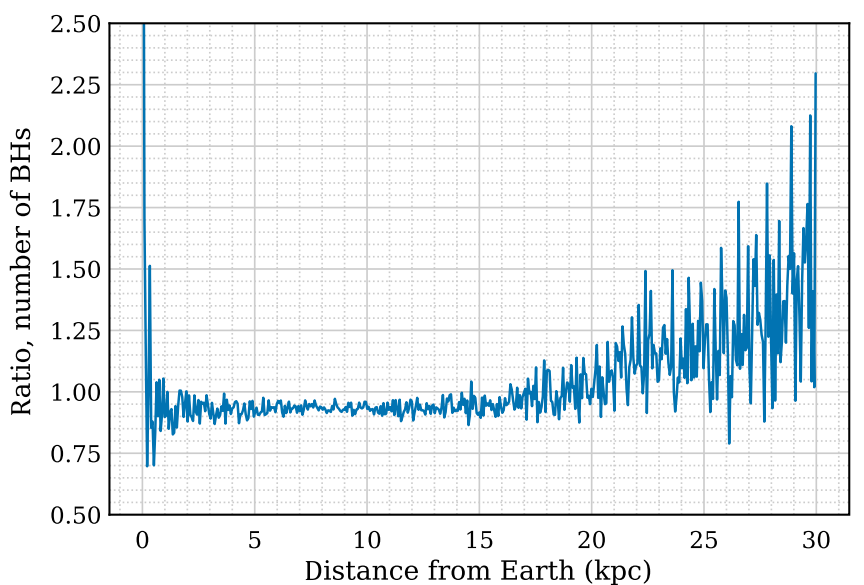

FIG. 24. If black holes are born with faster natal kicks (100 rather than $50 \mathrm{~km} / \mathrm{s}$ ), more of them have sufficiently high speeds to escape the gravitational pull of the Galactic bulge and disk. This causes a slight deficit of black holes at small Galactic radii, which also means there are fewer BHs close to Earth, decreasing on average the number of loud signals.

first stage of current all-sky continuous wave searches. This is true for both the standard $\left(M_{\mathrm{BH}, \max }=20 \mathrm{M}_{\odot}\right)$ and heavy $\left(M_{\mathrm{BH}, \max }=30 \mathrm{M}_{\odot}\right)$ populations. In both cases, the maximum $\dot{f}$ from the changing cloud mass is $6 \times 10^{-13} \mathrm{~Hz} / \mathrm{s}$.

\section{APPENDIX B: HIGHER NATAL KICKS}

Throughout this work, we focus on a black hole population with natal kicks given by a Maxwell-Boltzmann

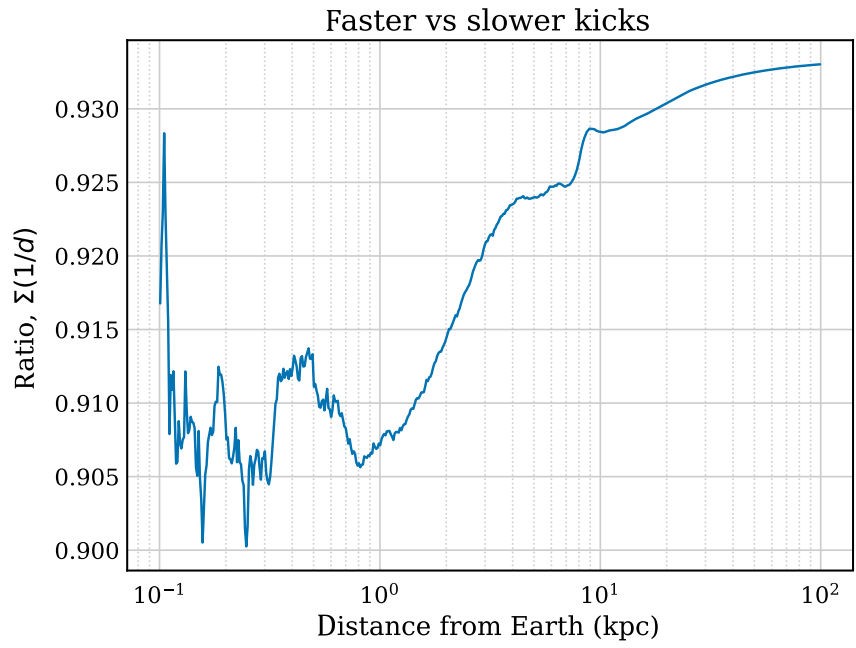

FIG. 25. The ratio of the sum of $1 / d$ for all the black holes within a distance $d$ provides a comparison of the number of signals between the two populations. Here we plot the ratio for the population with faster versus slower kicks. Overall, this ratio is smaller than unity and is decreasing toward shorter distances; the ratio is affected by small number statistics at distances much less than $1 \mathrm{kpc}$. The lower signal number is consistent with the fact that the population of black holes with faster kicks in general produces fewer signals above a given $h_{0}$ (Fig. 25).

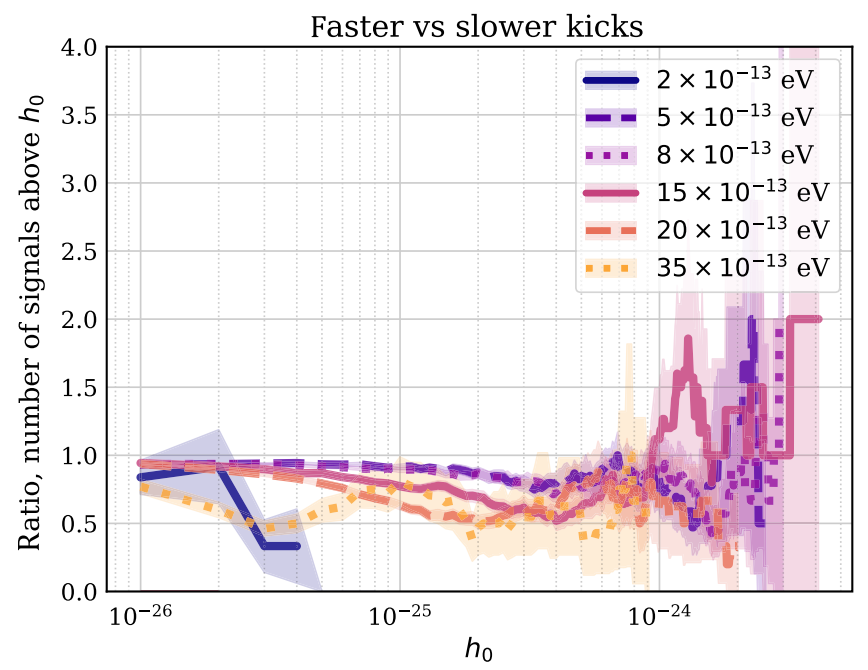

FIG. 26. The loudest signals in an ensemble are in general produced by the closest black holes. A black hole population with slightly faster natal kicks produces a slight deficit of boson clouds close to Earth (Fig. 24), resulting in fewer signals at larger values of $h_{0}$. This effect is greater for heavier bosons, as the louder signals are preferentially produced by closer black holes.

distribution with average 3D velocity of $50 \mathrm{~km} / \mathrm{s}$. Here we examine the ensemble signals that would be produced by black holes assuming they are born with average natal kicks of $100 \mathrm{~km} / \mathrm{s}$.

The black holes in the $100 \mathrm{~km} / \mathrm{s}$ population move faster on average, but the apparent $\dot{f}$ due to the proper motion is still many orders of magnitude smaller than that resolvable by continuous wave searches and is not a concern.

Black holes born with faster natal kicks are more likely to have sufficiently high speeds to escape the Galactic

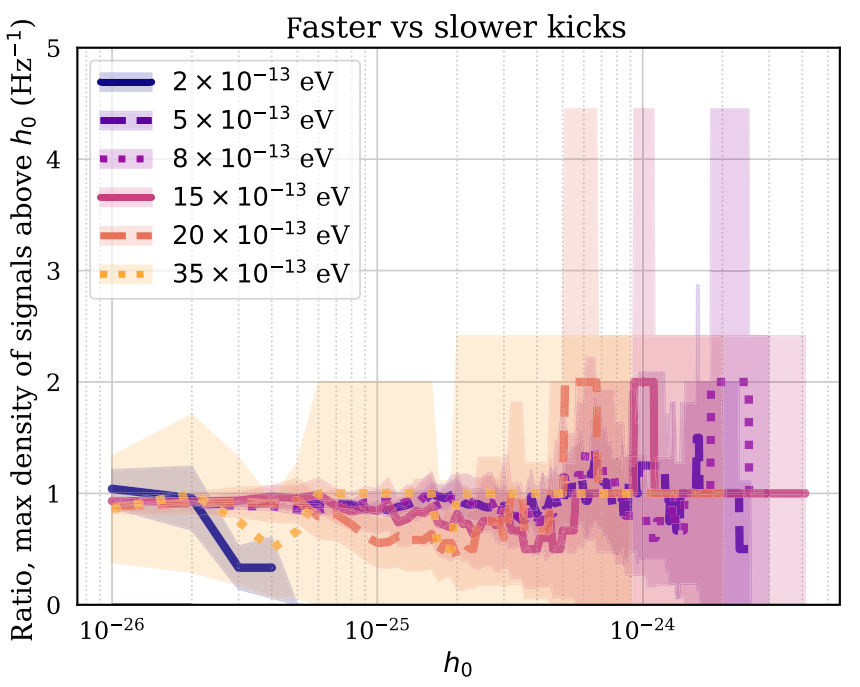

FIG. 27. The ratio of maximum signal density above a given $h_{0}$ between the faster and slower kick populations is consistent with unity. 


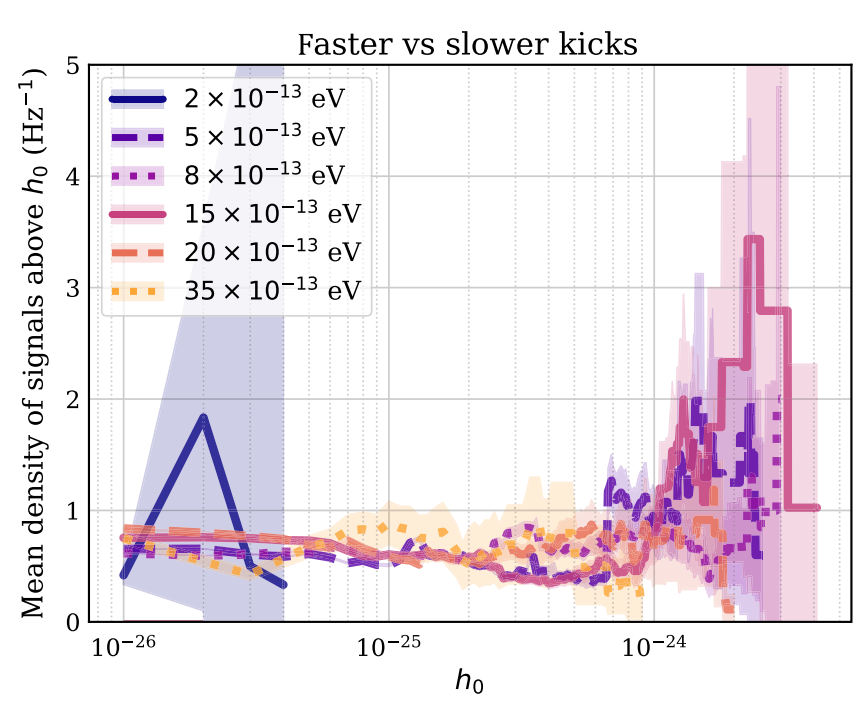

FIG. 28. The ratio of mean signal density above a given $h_{0}$ between the faster and slower kick populations reflects the number of signals above a given $h_{0}$ (Fig. 26). In general, black holes with faster natal kicks produce signals with larger Doppler shifts, thereby increasing the frequency range spanned by the ensemble and decreasing the mean signal density further.

bulge and disk and travel farther on average. Thus, they are more likely to be found at larger distances from both the Galactic Center and Earth (Fig. 24). Figure 25 shows the ratio of the sum of $1 / d$ for all the black holes within a distance $d$; this quantity is smaller than 1 and decreases with decreasing distance from Earth until very small distances, where it is dominated by small number fluctuations. The ratio of the sum of $1 / d$ approximates Fig. 26, the ratio of the number of signals above a given value of $h_{0}$, which is in general less than 1 and decreases with increasing $h_{0}$ until the loudest signals $\left(h_{0}>10^{-24}\right)$, a regime that is dominated by the few closest black holes.

The smaller number of signals above a given $h_{0}$ for the population with larger natal kicks also produces smaller maximum and mean densities on average (Figs. 27 and 28).

\section{APPENDIX C: LIGHT BLACK HOLE POPULATION}

We compare the ensemble signals from the standard black hole population $\left(M_{\mathrm{BH}} \in[5,20] \mathrm{M}_{\odot}\right)$ with the ensemble signals from a lighter black hole population $\left(M_{\mathrm{BH}} \in[3,20] \mathrm{M}_{\odot}\right)$. We maintain the distribution shape (Salpeter function) as well as the total number of black holes $\left(10^{8}\right)$; reducing the minimum black hole mass therefore reduces the number of black holes of all other masses.

For $\mu_{\mathrm{b}}=3.5 \times 10^{-12} \mathrm{eV}$, the addition of the lighter black holes results in a larger number of signals with $h_{0} \lesssim 3 \times 10^{-25}$; a similar but smaller effect is seen for $\mu_{\mathrm{b}}=2 \times 10^{-12} \mathrm{eV}$. This is due to the fact that at these

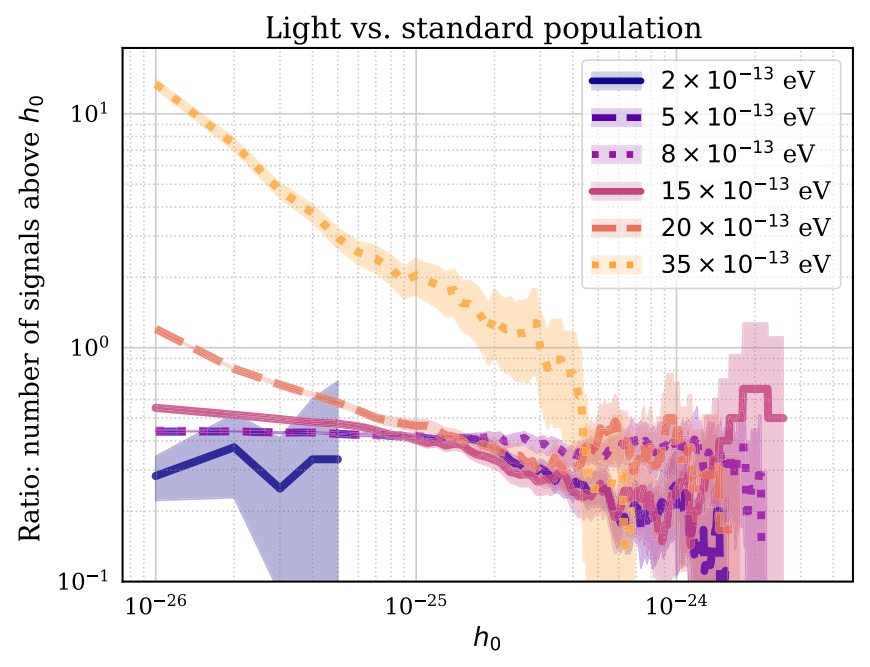

FIG. 29. Increasing the number of light black holes $\left(M_{\mathrm{BH}}<5 \mathrm{M}_{\odot}\right)$ while maintaining the same number of black holes effectively reduces the number of black holes at all other masses. Therefore, the number of signals above a given $h_{0}$ decreases by a factor of 2 for most of the boson masses. The exception is the heaviest boson $\left(\mu_{\mathrm{b}}=3.5 \times 10^{-12} \mathrm{eV}\right)$, for which the number of signals is larger with the light black hole population for signals with $h_{0}<3 \times 10^{-25}$.

heavy boson masses, lighter black holes can more easily satisfy the critical spin condition while still producing relatively large signals. However, the current continuous wave search upper limits are around $10^{-24}$ near the signal frequencies produced by these heavy bosons, so the addition of the lighter black holes does not affect our estimates of the number of signals detectable by current continuous wave searches.

The overall effect is that the total number of signals decreases by about a factor of 2 for all but the heaviest boson masses (Fig. 29). In reality, the black hole mass distribution should turn over at the lightest masses, i.e., the majority of black holes are still expected to have mass above $5 \mathrm{M}_{\odot}[98,113]$. Therefore, Fig. 29 can be considered a lower bound on the ratio of the number of signals for the case in which black holes lighter than $5 \mathrm{M}_{\odot}$ exist in the Galaxy.

\section{APPENDIX D: HEAVY BLACK HOLE POPULATION}

We examine the ensemble signal for a heavy population of black holes, with $M_{\mathrm{BH} \text {,max }}=30 \mathrm{M}_{\odot}$ and compare the resultant ensemble signals with the signals from the standard population.

We show the ratio of the heavy to the standard population in the number of signals (Fig. 30), mean density of signals (Fig. 31), and maximum density of signals (Fig. 32). The ratios are generally consistent with 1 . The exceptions are the number and density of signals for the lightest boson 


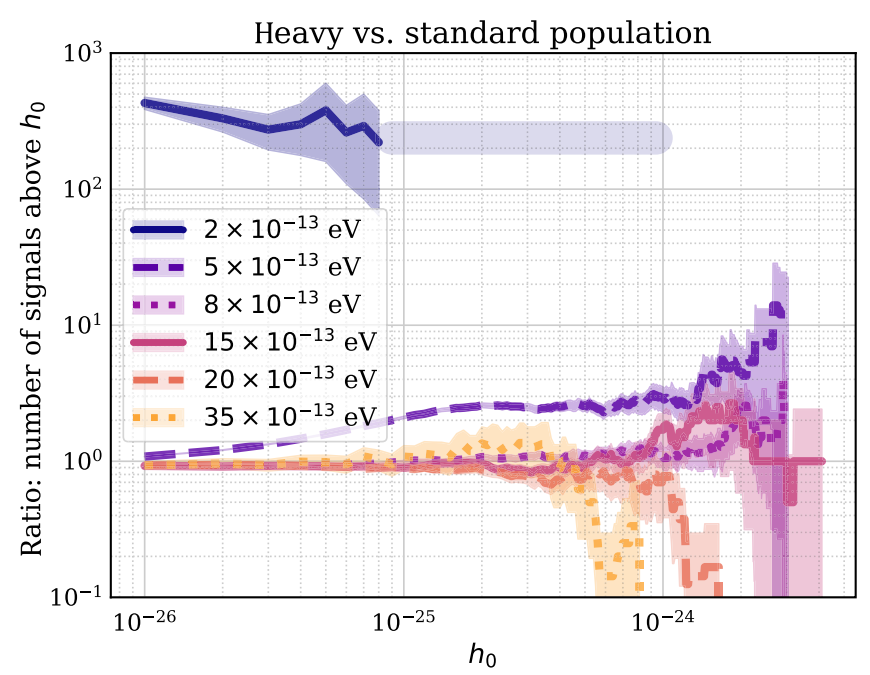

FIG. 30. The ratio of the number of signals above a given $h_{0}$ for the heavy $\left(M_{\mathrm{BH}, \max }=30 \mathrm{M}_{\odot}\right)$ versus standard $\left(M_{\mathrm{BH}, \max }=20 \mathrm{M}_{\odot}\right)$ populations. The number of signals for the lighter bosons $\left(\mu_{\mathrm{b}}=2 \times 10^{-13}\right.$ and $\left.5 \times 10^{-13} \mathrm{eV}\right)$ increases in going from the standard to the heavy black hole population, while the number of signals for the heavier bosons stays approximately the same (Sec. VA). For $\mu_{\mathrm{b}}=2 \times 10^{-13} \mathrm{eV}$, the signals from the heavy black hole population extend to strains of $h_{0} \approx 10^{-24}$ (indicated by the light, thick bar) while the signals from the standard black hole population only have $h_{0} \leq 10^{-25}$.

mass of $2 \times 10^{-13} \mathrm{eV}$, which is a factor of several hundred larger for the heavy population, and for $5 \times 10^{-13} \mathrm{eV}$, a factor of approximately 2 larger. In addition, the mean density of signals is reduced by $\sim 20 \%$ for the intermediate boson mass of $8 \times 10^{-13} \mathrm{eV}$ due to the larger range of

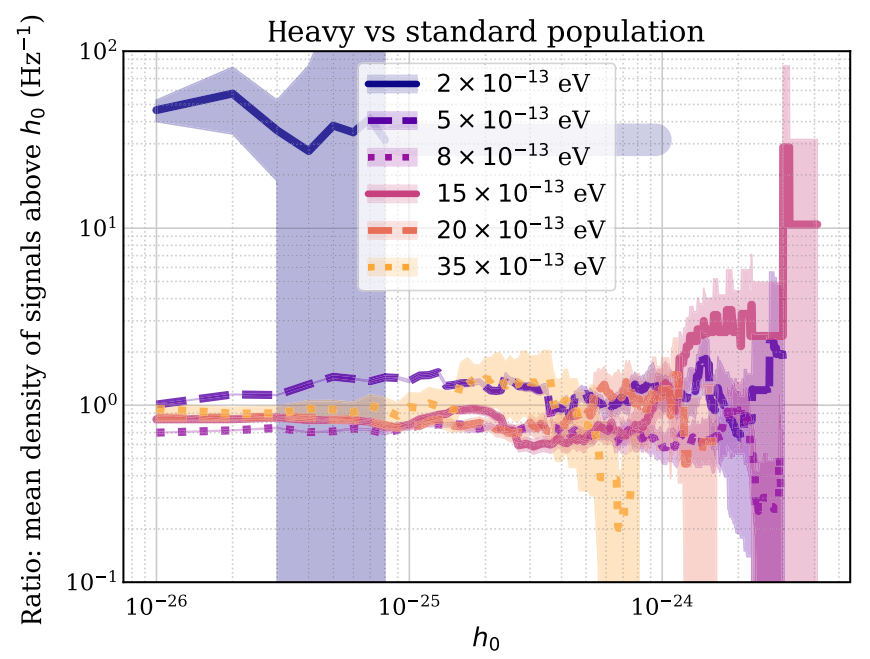

FIG. 31. The ratio of the mean signal density above a given $h_{0}$ for the heavy versus standard black hole populations is approximately unity for the five heavier boson masses. For the lightest mass $\left(\mu_{\mathrm{b}}=2 \times 10^{-13} \mathrm{eV}\right)$, the mean density is over 10 times larger for the heavier black hole population.

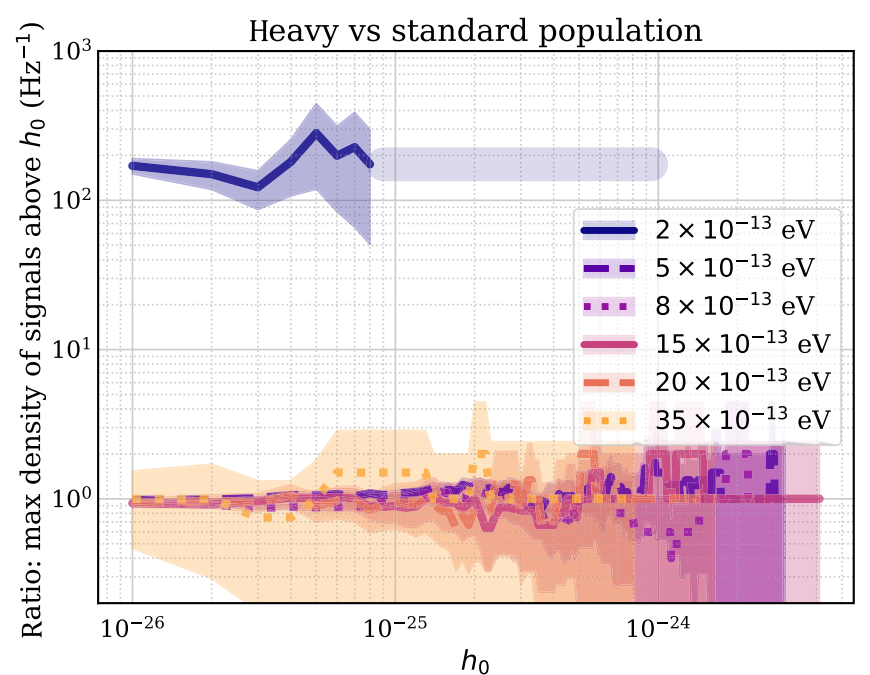

FIG. 32. For the six boson masses plotted here, the ratio of the maximum signal density above a given $h_{0}$ for the heavy versus standard black hole populations is similar to the ratio of the mean signal density. The maximum signal density for the lightest boson $\left(\mu_{\mathrm{b}}=2 \times 10^{-13} \mathrm{eV}\right)$ is over 100 times larger for the heavier black hole population.

frequencies covered by the heavier black hole population for a fixed black hole number.

We also show the mass and spin distributions (Fig. 33) as well as the age and distance distributions (Fig. 34) for the heavy black hole population. In the heavy population, black holes from farther away are able to source loud signals relative to the standard population.

\section{APPENDIX E: ENSEMBLE SIGNAL: SIGNALS ABOVE THE DETECTION THRESHOLD}

As discussed in Sec. VB, large signal numbers and densities can affect the search efficiency. Figures 35-42 show the number of signals, as well as the maximum and average strain of signals, with amplitude $h_{0}$ above the upper limit values set by two recent continuous wave allsky searches, Freq Hough [22] and Falcon [20,21,114] for boson masses between $3 \times 10^{-13}$ and $1.5 \times 10^{-12} \mathrm{eV}$. We consider both standard and heavy black hole mass distributions, and both standard and moderate spin distributions.

\section{APPENDIX F: CONTRIBUTION TO THE NOISE AMPLITUDE SPECTRAL DENSITY FROM THE ENSEMBLE SIGNAL}

As discussed in Sec. V B, the amplitude spectral density can be significantly altered from search expectations by the ensemble signal. Figures 43-47 show the expected amplitude spectral density of the ensemble signal from a Fourier transform of varying time baseline $\left(T_{S F T}\right)$, under different assumptions on, in particular, the maximum black hole 

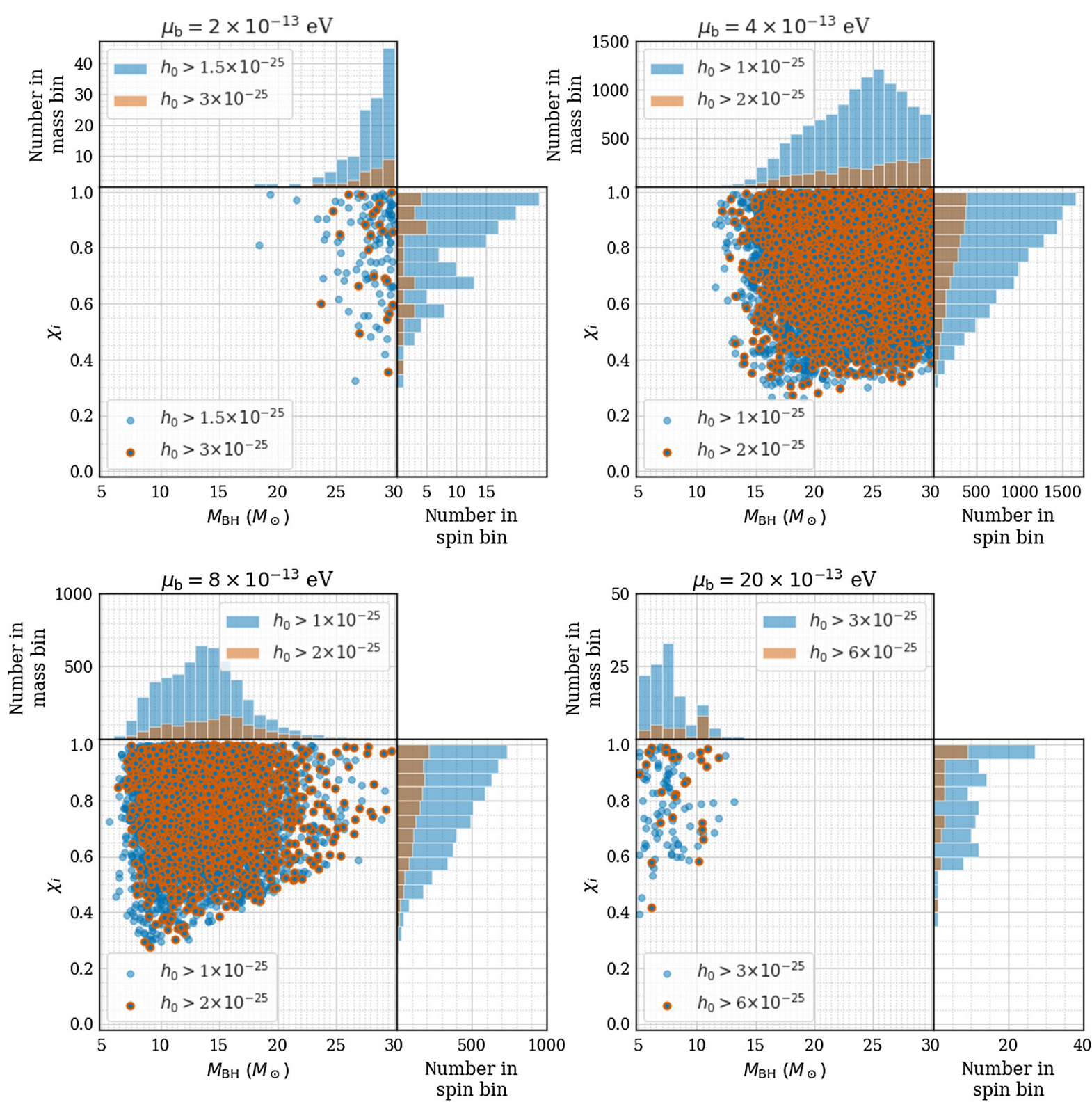

FIG. 33. Mass and spin properties of the heavy black hole population. For the two heavier boson masses $\left(\mu_{\mathrm{b}}=8 \times 10^{-13}\right.$ and $2 \times 10^{-12} \mathrm{eV}$, the black holes that produce the potentially detectable ensemble signals have similar properties to the black holes that produce the signals in Fig. 10 (the standard population) due to the fact that systems with large values of $\alpha$ have dramatically shortened lifetimes. For the two lighter boson masses $\left(\mu_{\mathrm{b}}=2 \times 10^{-13}\right.$ and $\left.4 \times 10^{-13} \mathrm{eV}\right)$, the addition of black holes with $M_{\mathrm{BH}}>20 \mathrm{M}_{\odot}$ greatly increases the number of detectable signals. 

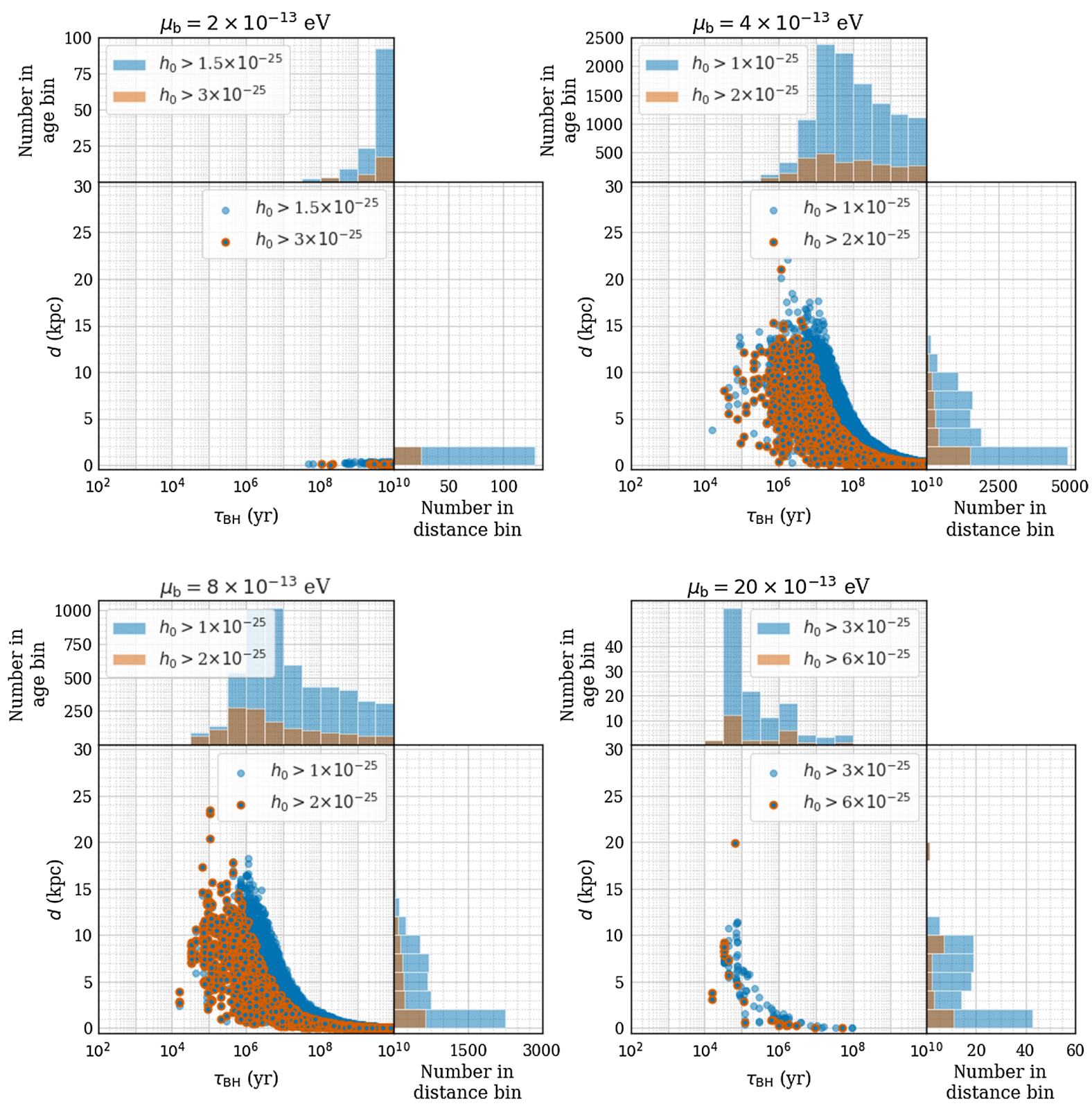

FIG. 34. Ages and distances of the heavy black hole population. As in Fig. 33, the addition of black holes with $M_{\mathrm{BH}}>20 \mathrm{M}_{\odot}$ increases the number of detectable systems for the lighter bosons $\left(\mu_{\mathrm{b}}=2 \times 10^{-13}\right.$ and $\left.4 \times 10^{-13} \mathrm{eV}\right)$ but has little to no effect on the heavier bosons. 

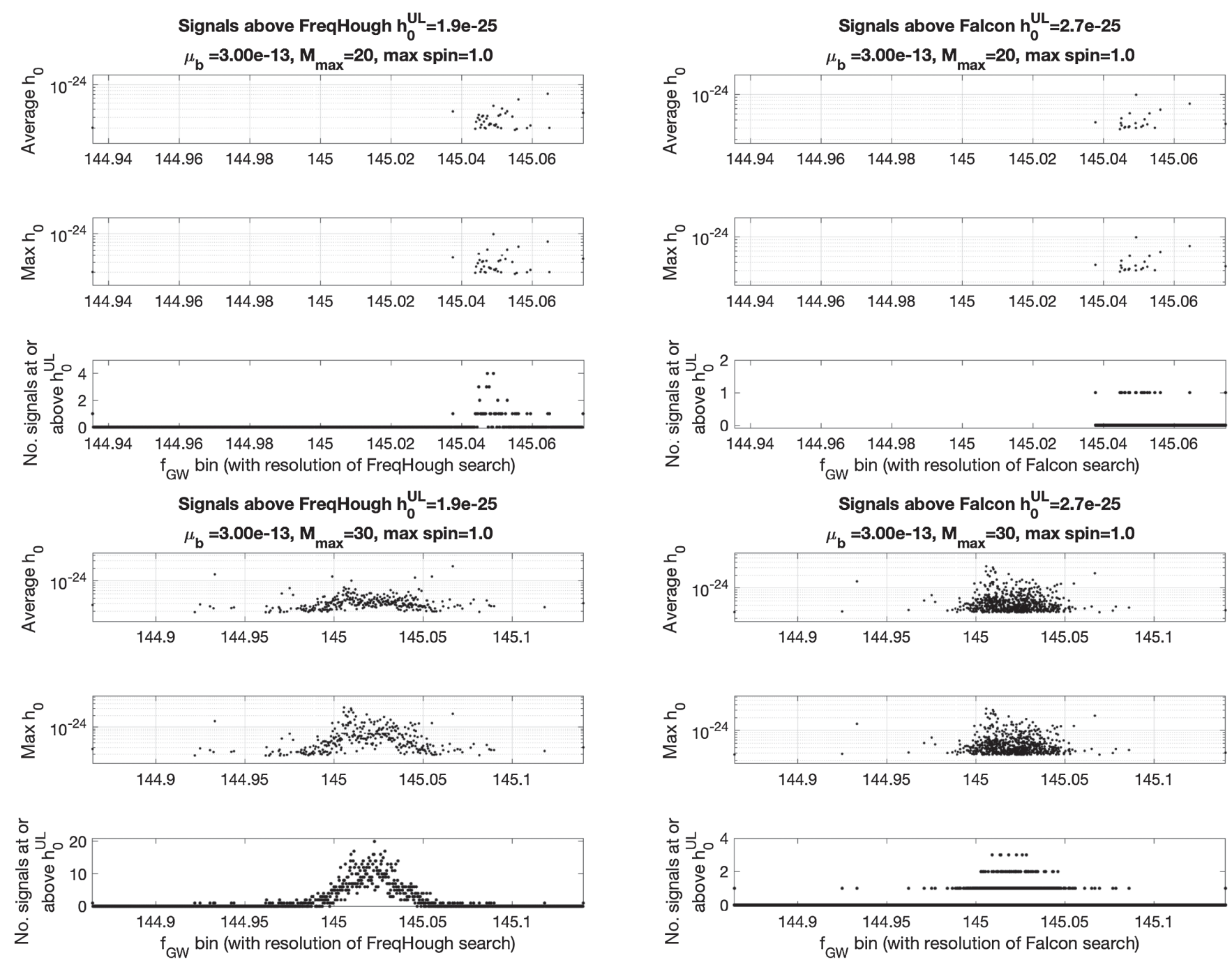

FIG. 35. The $x$ axes show the signal frequency binned with the resolution of the two searches: (left panels) Freq Hough, $2.4 \times 10^{-4} \mathrm{~Hz}$ [22] and (right panels) Falcon, $1.7 \times 10^{-5} \mathrm{~Hz}$ [20,21]. From the bottom panel up, the $y$ axis shows the number of detectable signals in each bin, their maximum intrinsic amplitude, and their average amplitude. 

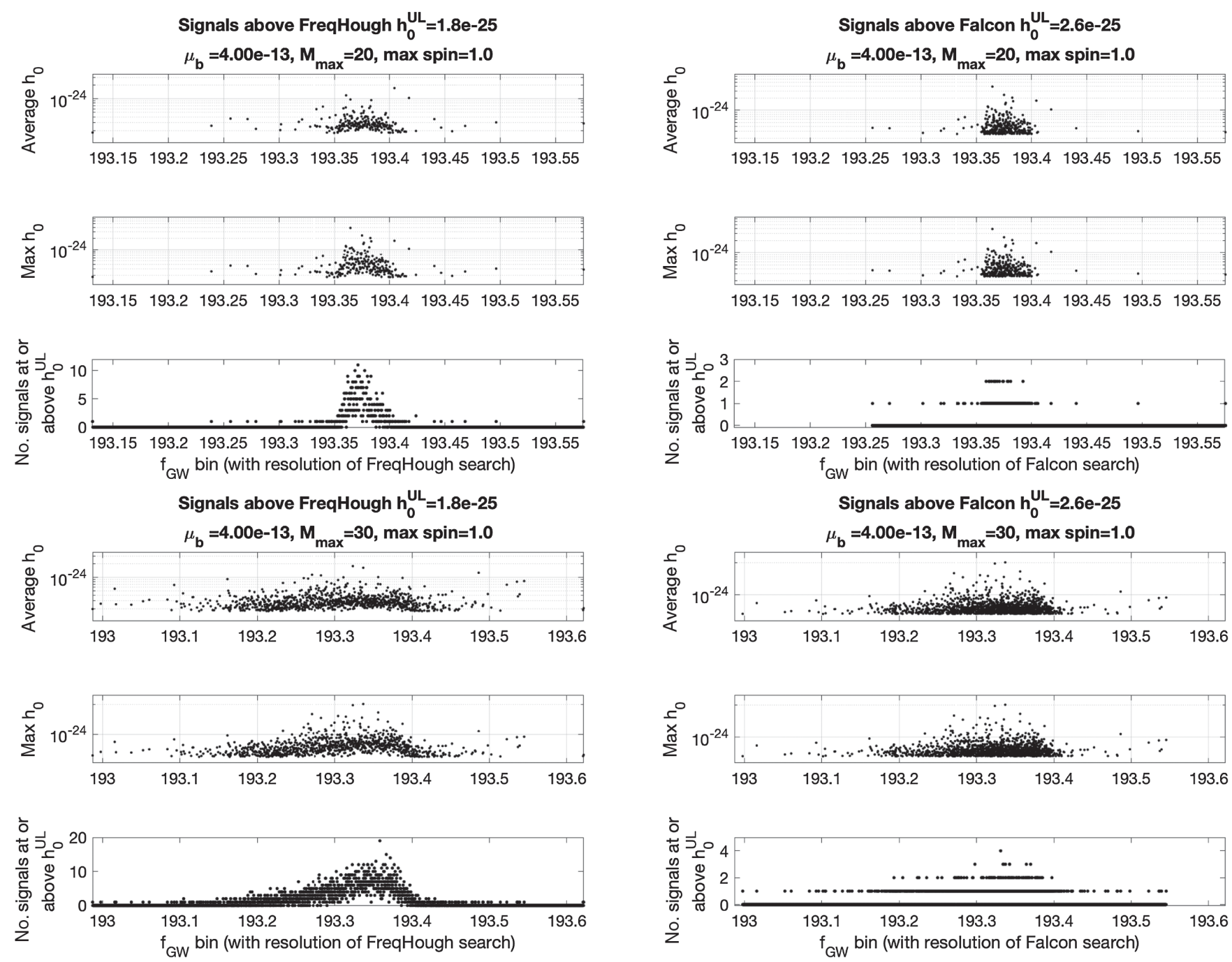

FIG. 36. The $x$ axes show the signal frequency binned with the resolution of the two searches: (left panels) Freq Hough, $2.4 \times 10^{-4} \mathrm{~Hz}$ [22] and (right panels) Falcon [20,21], $1.7 \times 10^{-5} \mathrm{~Hz}$. From the bottom panel up, the $y$ axis shows the number of detectable signals in each bin, their maximum intrinsic amplitude, and their average amplitude. 

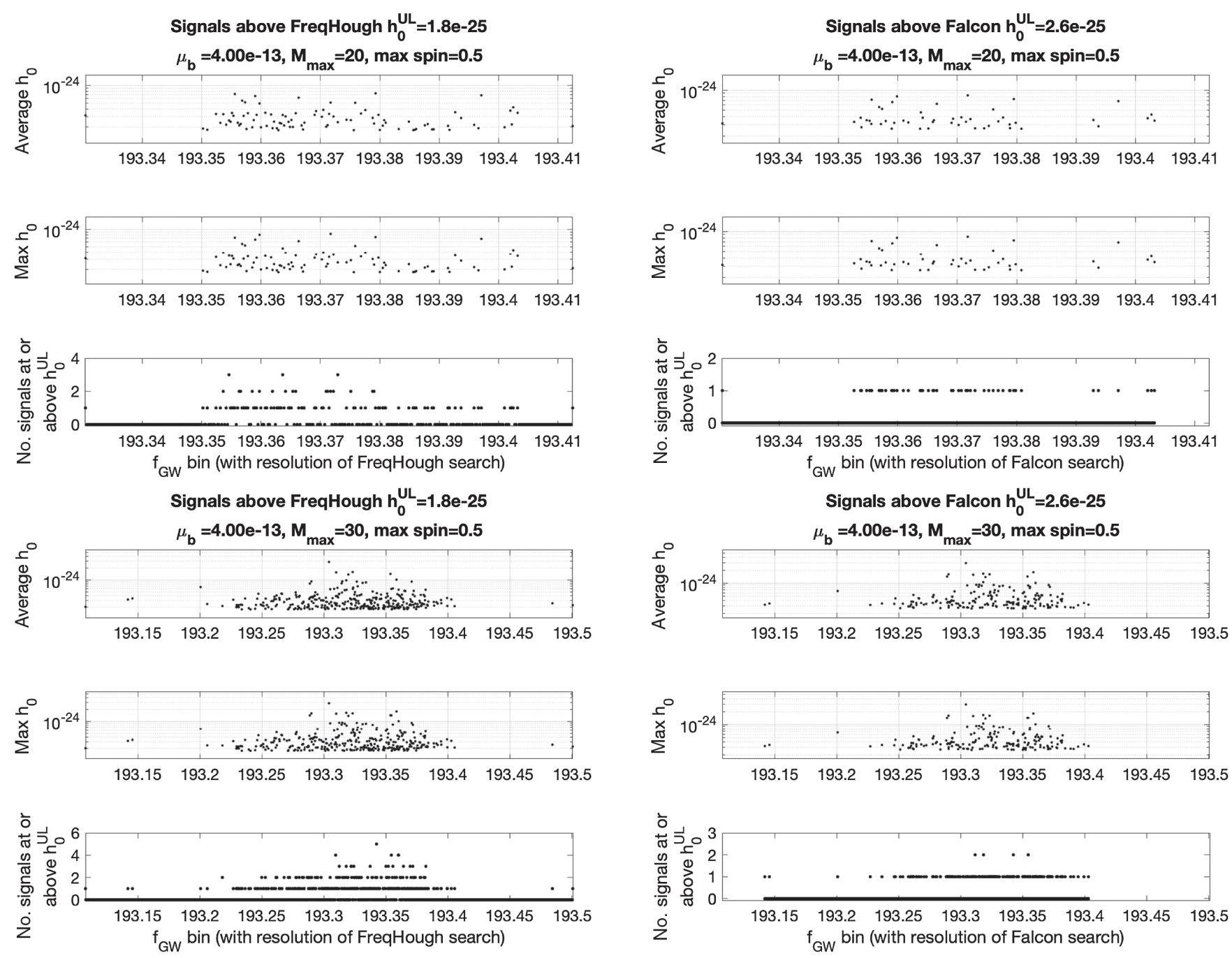

FIG. 37. The $x$-axis plots show the signal frequency binned with the resolution of the two searches: (left panels) Freq Hough, $2.4 \times 10^{-4} \mathrm{~Hz}$ [22] and (right panels) Falcon [20,21], $1.7 \times 10^{-5} \mathrm{~Hz}$. From the bottom panel up, the $y$ axis shows the number of detectable signals in each bin, their maximum intrinsic amplitude, and their average amplitude. 

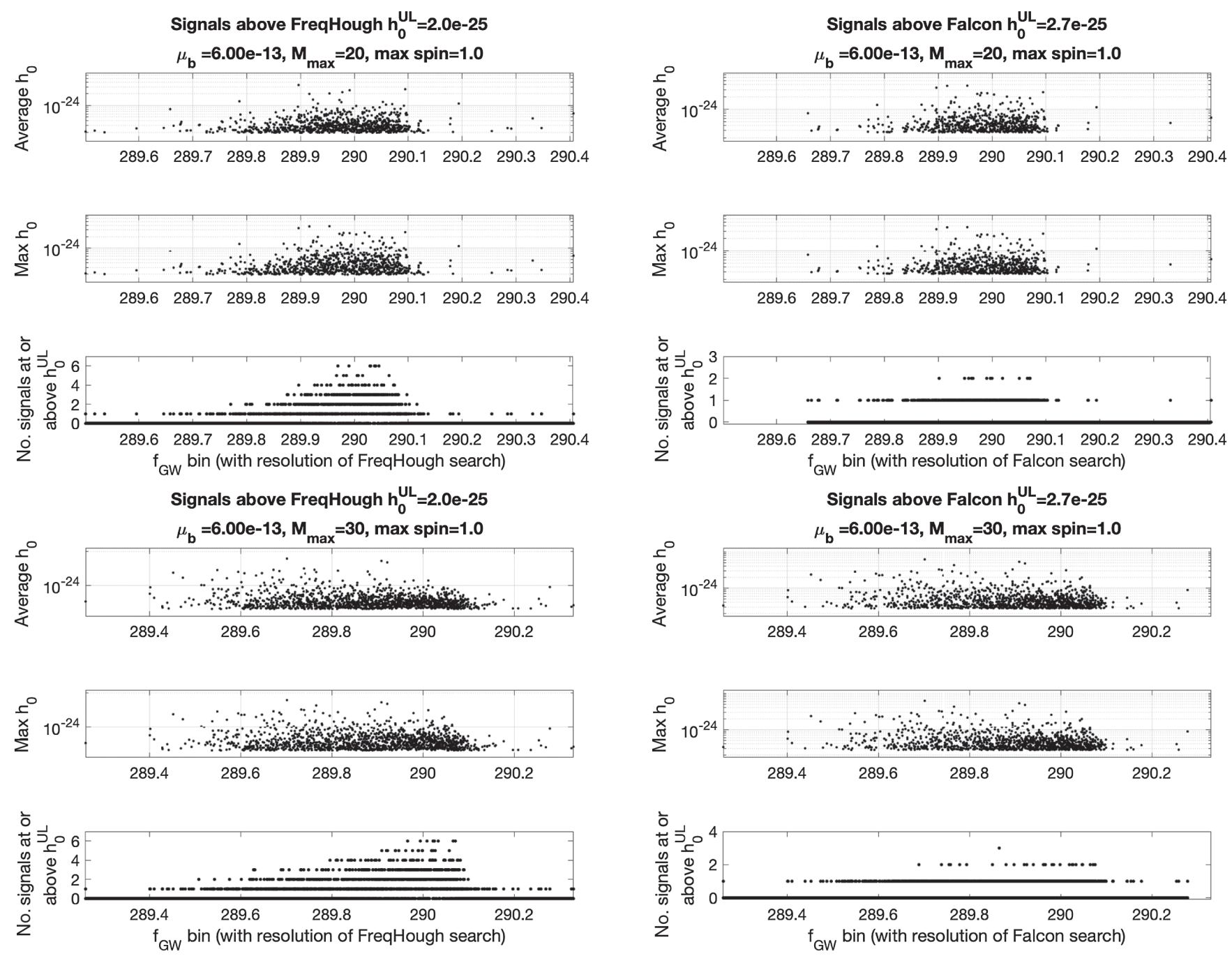

FIG. 38. The $x$ axes show the signal frequency binned with the resolution of the two searches: (left panels) Freq Hough, $2.4 \times 10^{-4} \mathrm{~Hz}$ [22] and (right panels) Falcon [20,21], $1.7 \times 10^{-5} \mathrm{~Hz}$. From the bottom panel up, the $y$ axis shows the number of detectable signals in each bin, their maximum intrinsic amplitude, and their average amplitude. 

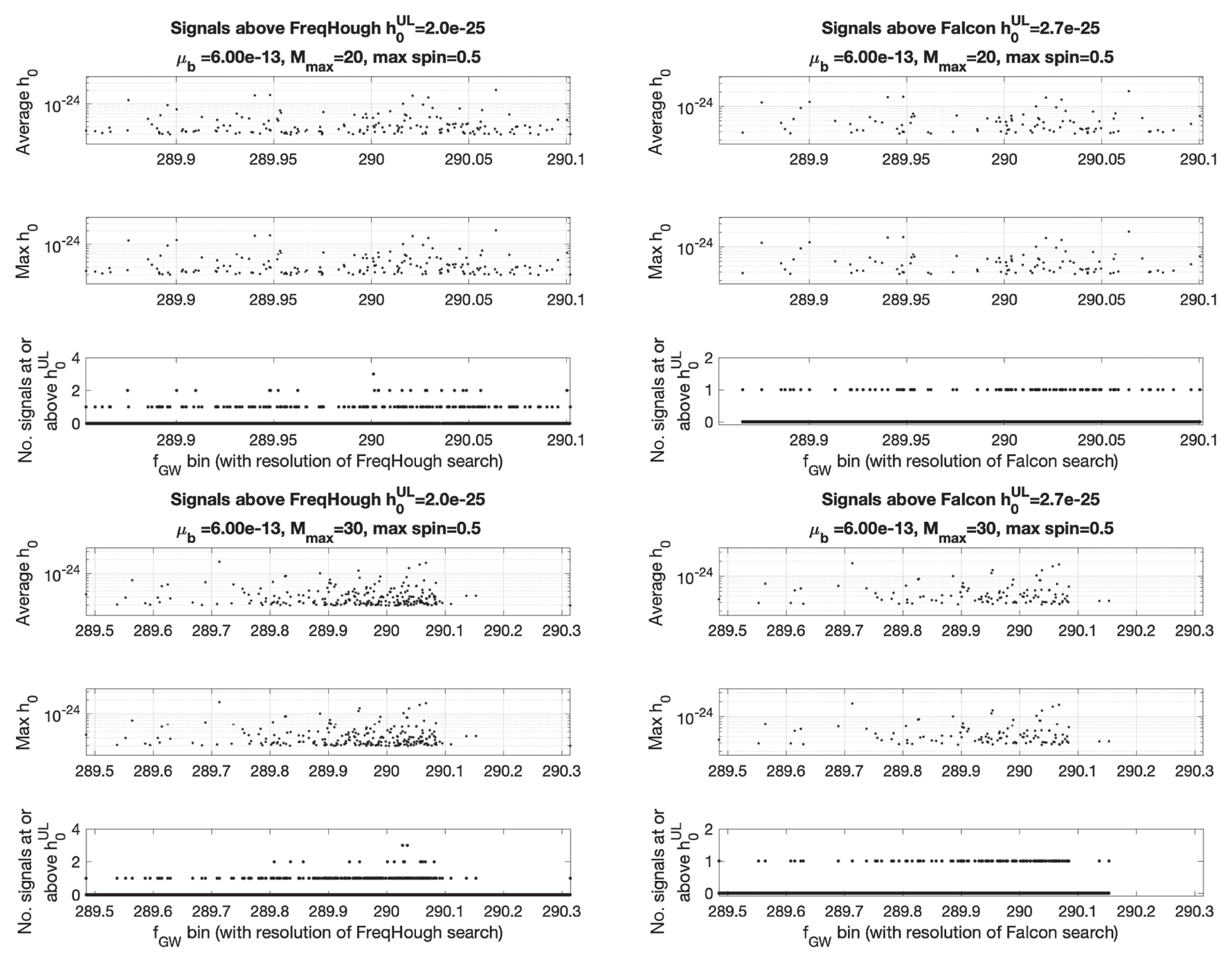

FIG. 39. The $x$ axes show the signal frequency binned with the resolution of the two searches: (left panels) Freq Hough, $2.4 \times 10^{-4} \mathrm{~Hz}$ [22] and (right panels) Falcon, $1.7 \times 10^{-5} \mathrm{~Hz}$ [20,21]. From the bottom panel up, the $y$ axis shows the number of detectable signals in each bin, their maximum intrinsic amplitude, and their average amplitude. 

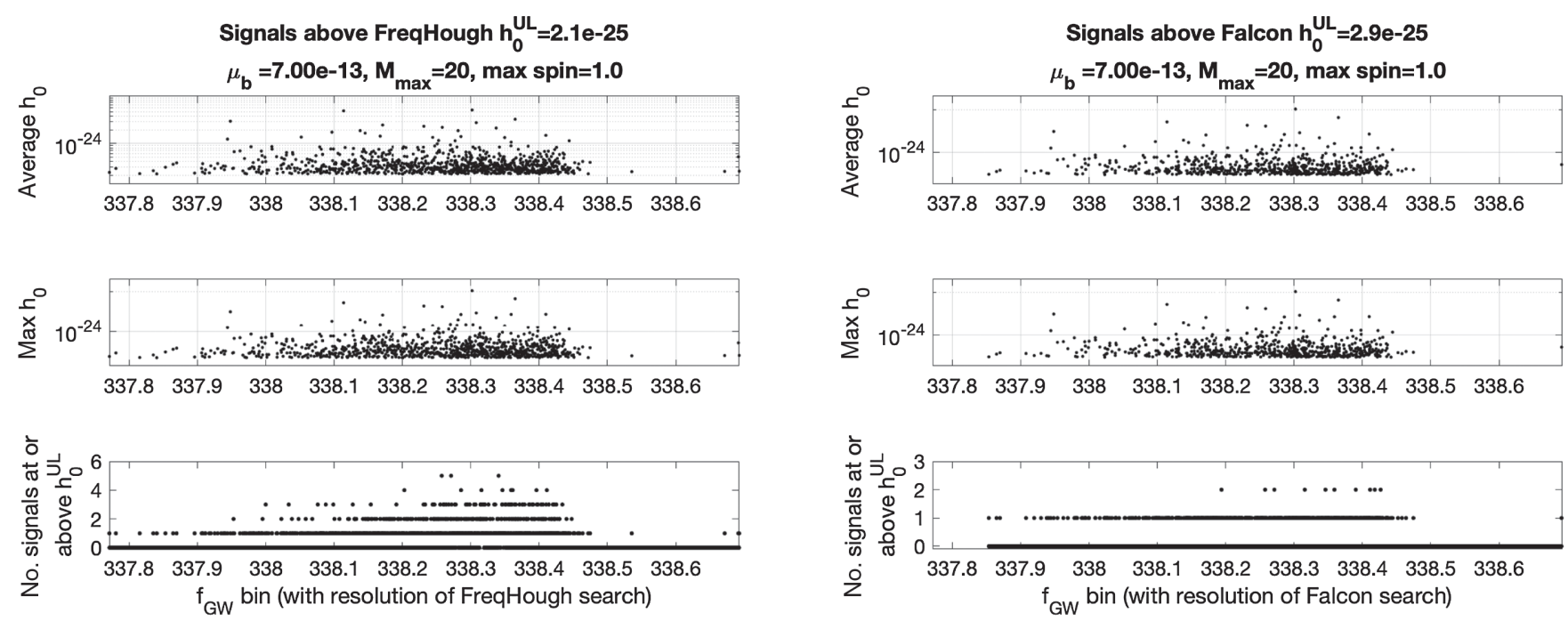

Signals above FreqHough $h_{0}^{\mathrm{UL}}=2.1 \mathrm{e}-25$
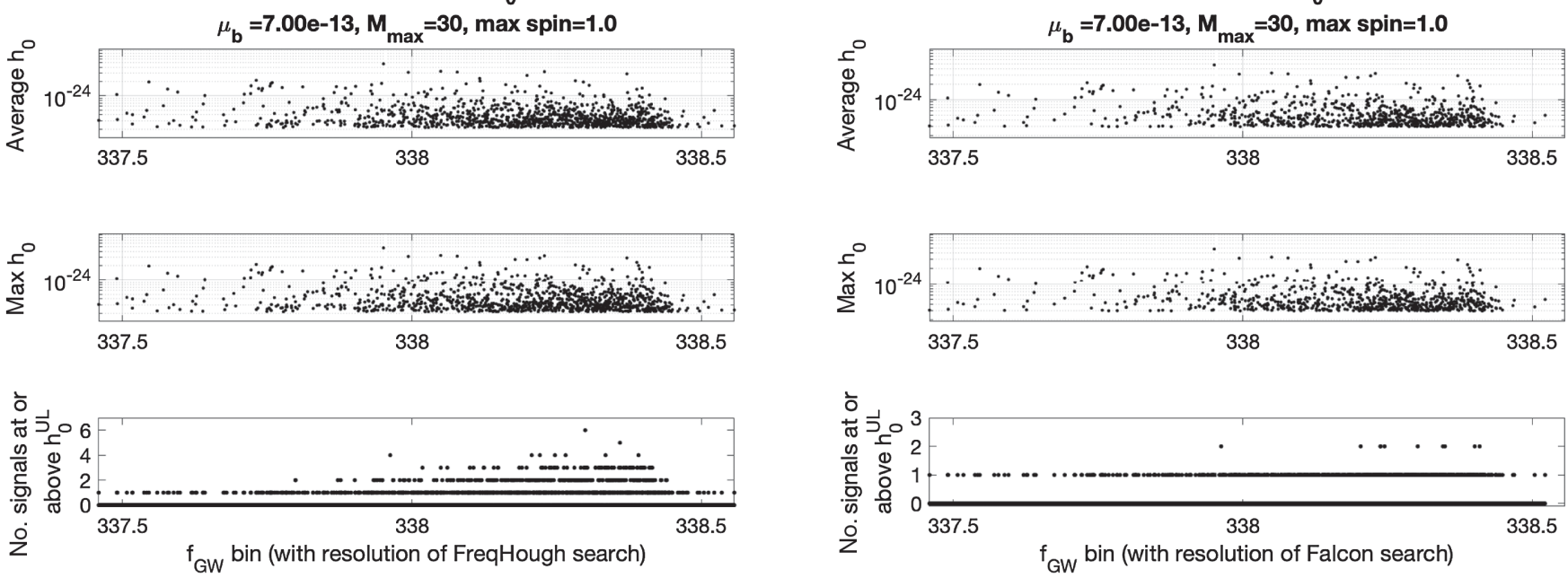

FIG. 40. The $x$ axes show the signal frequency binned with the resolution of the two searches: (left panels) Freq Hough, $2.4 \times 10^{-4} \mathrm{~Hz}$ [22] and (right panels) Falcon, $1.7 \times 10^{-5} \mathrm{~Hz}$ [20,21]. From the bottom panel up, the $y$ axis shows the number of detectable signals in each bin, their maximum intrinsic amplitude, and their average amplitude. 

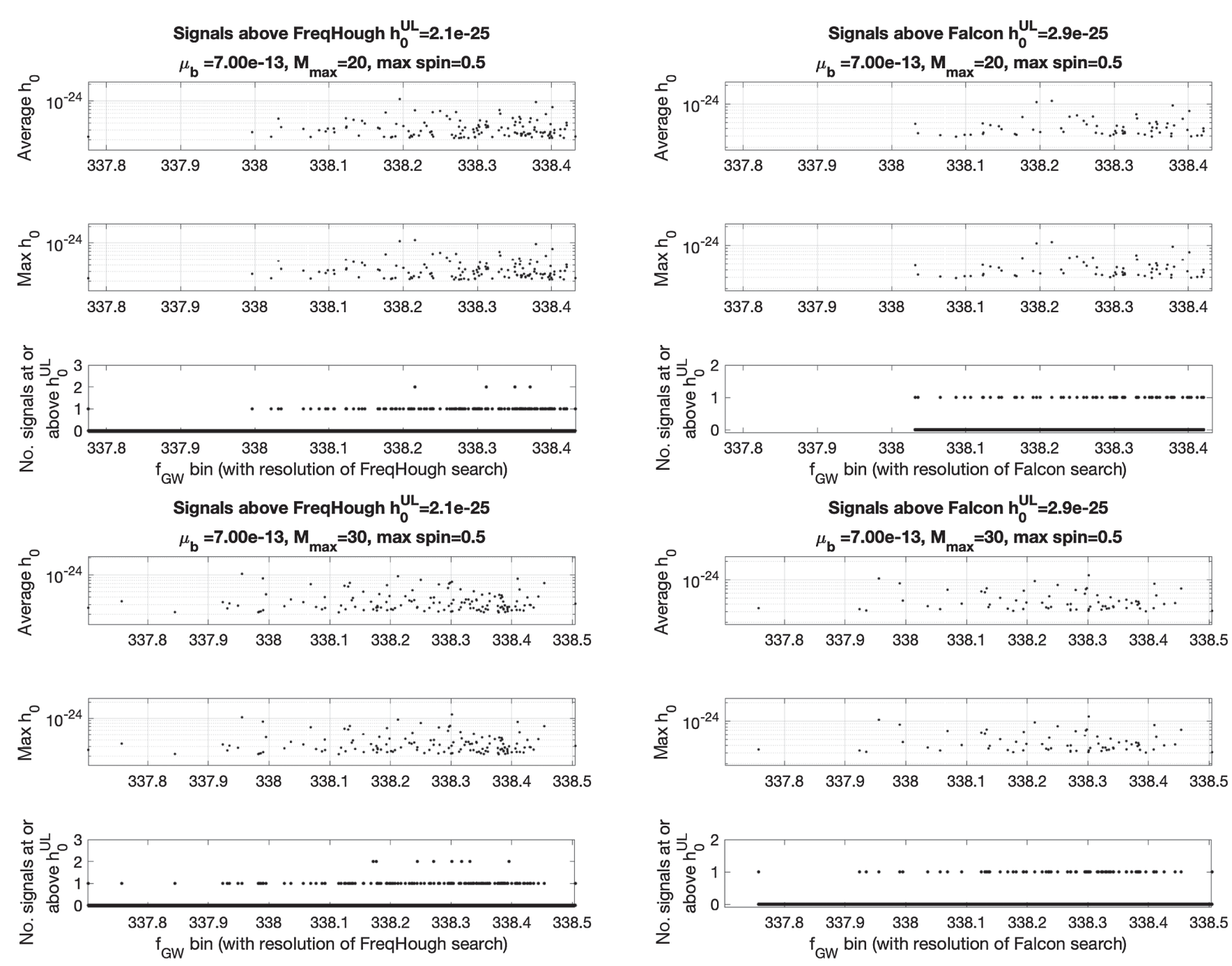

FIG. 41. The $x$ axes show the signal frequency binned with the resolution of the two searches: (left panels) Freq Hough, $2.4 \times 10^{-4} \mathrm{~Hz}$ [22] and (right panels) Falcon, $1.7 \times 10^{-5} \mathrm{~Hz}$ [20,21]. From the bottom panel up, the $y$ axis shows the number of detectable signals in each bin, their maximum intrinsic amplitude, and their average amplitude. 

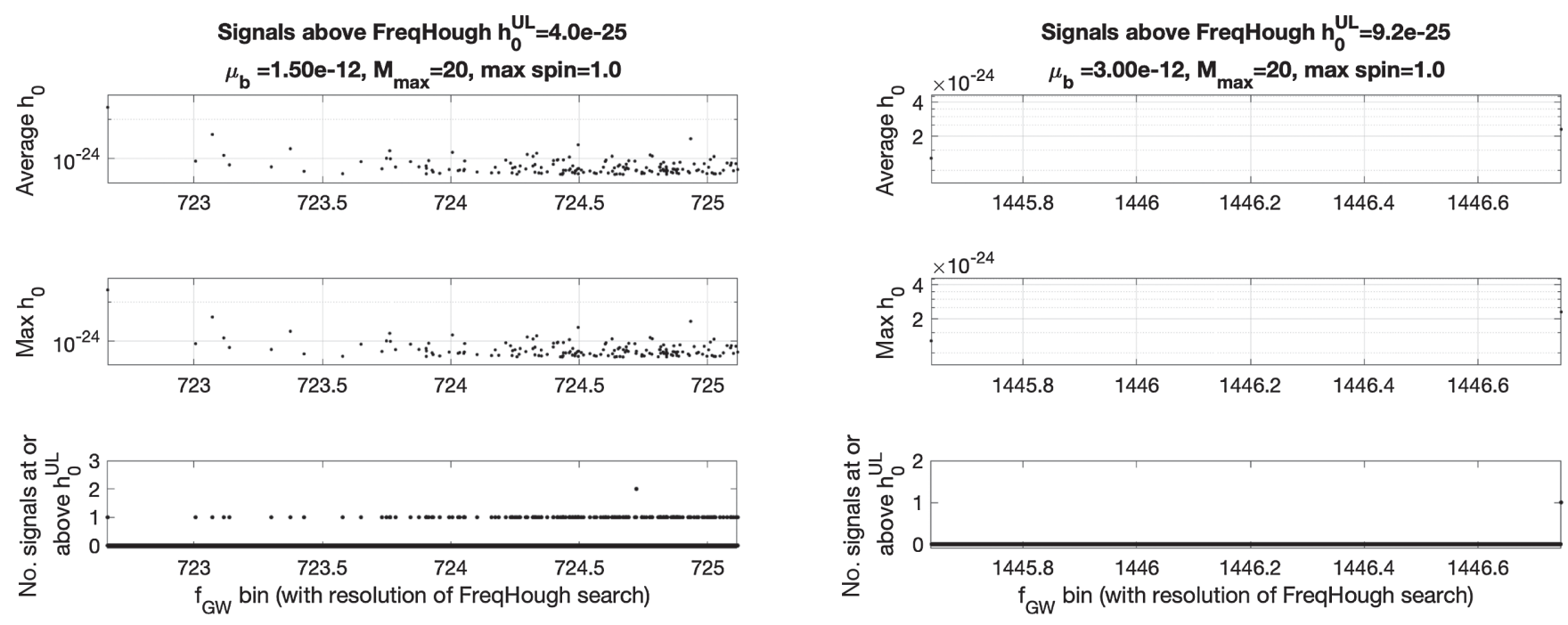

Signals above FreqHough $h_{0}^{\mathrm{UL}}=4.0 \mathrm{e}-25$

Signals above FreqHough $h_{0}^{\mathrm{UL}}=9.2 \mathrm{e}-25$
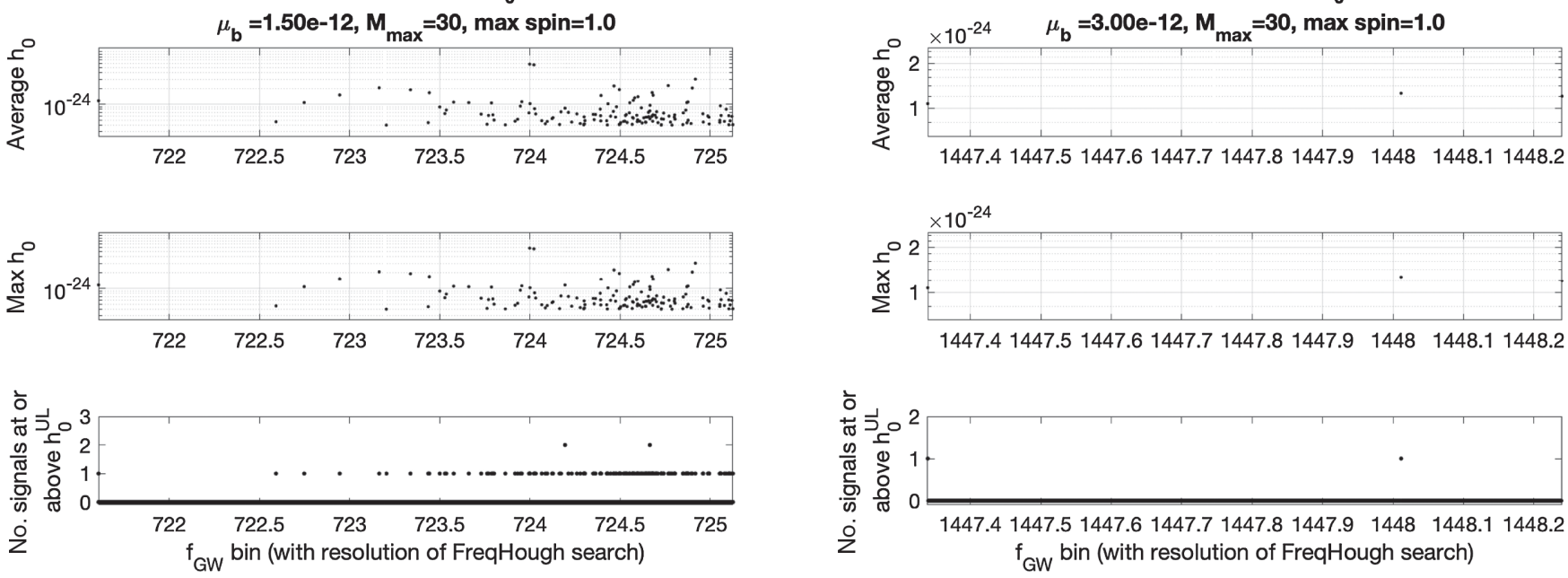

FIG. 42. The $x$ axes show the signal frequency binned with the resolution of the two searches: (left panels) Freq Hough, $2.4 \times 10^{-4} \mathrm{~Hz}$ [22] and (right panels) Falcon, $1.7 \times 10^{-5} \mathrm{~Hz}$ [20,21]. From the bottom panel up, the $y$ axis shows the number of detectable signals in each bin, their maximum intrinsic amplitude, and their average amplitude. 

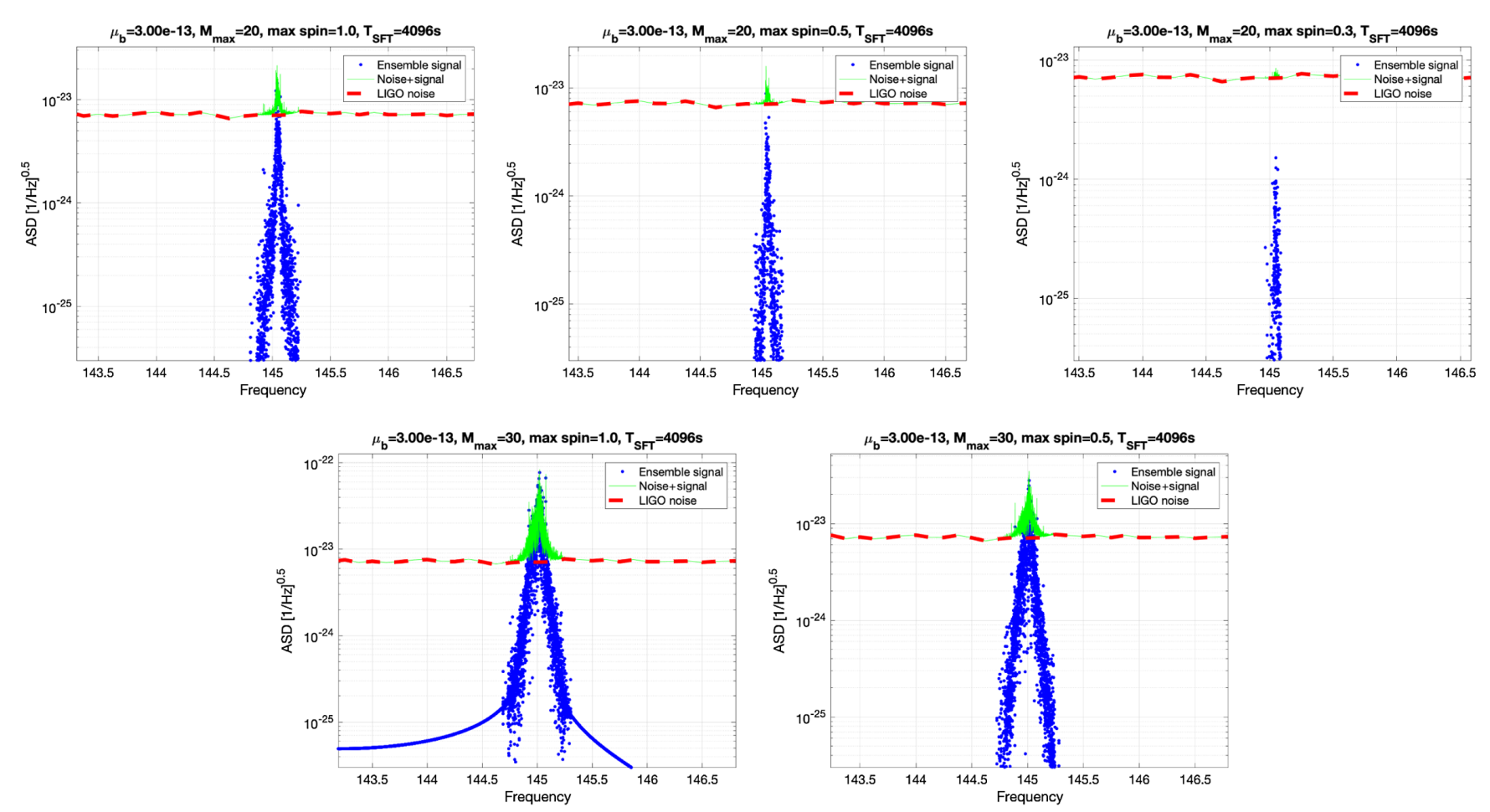

FIG. 43. The amplitude spectral density of the LIGO O2 data alone (dashed red line) and of an ensemble signal (top green points) assuming that $\mu_{\mathrm{b}}=3 \times 10^{-13} \mathrm{eV}$ and a Galactic black hole population with maximum mass $20 \mathrm{M}_{\odot}$ (top plots) and max initial spin of $0.3,0.5$ and 1 , and maximum mass $30 \mathrm{M}_{\odot}$ (bottom plots) and maximum initial spins of 0.5 and 1 . The time baseline assumed is $4096 \mathrm{~s}$, as used by the Freq Hough search in this frequency range [22].
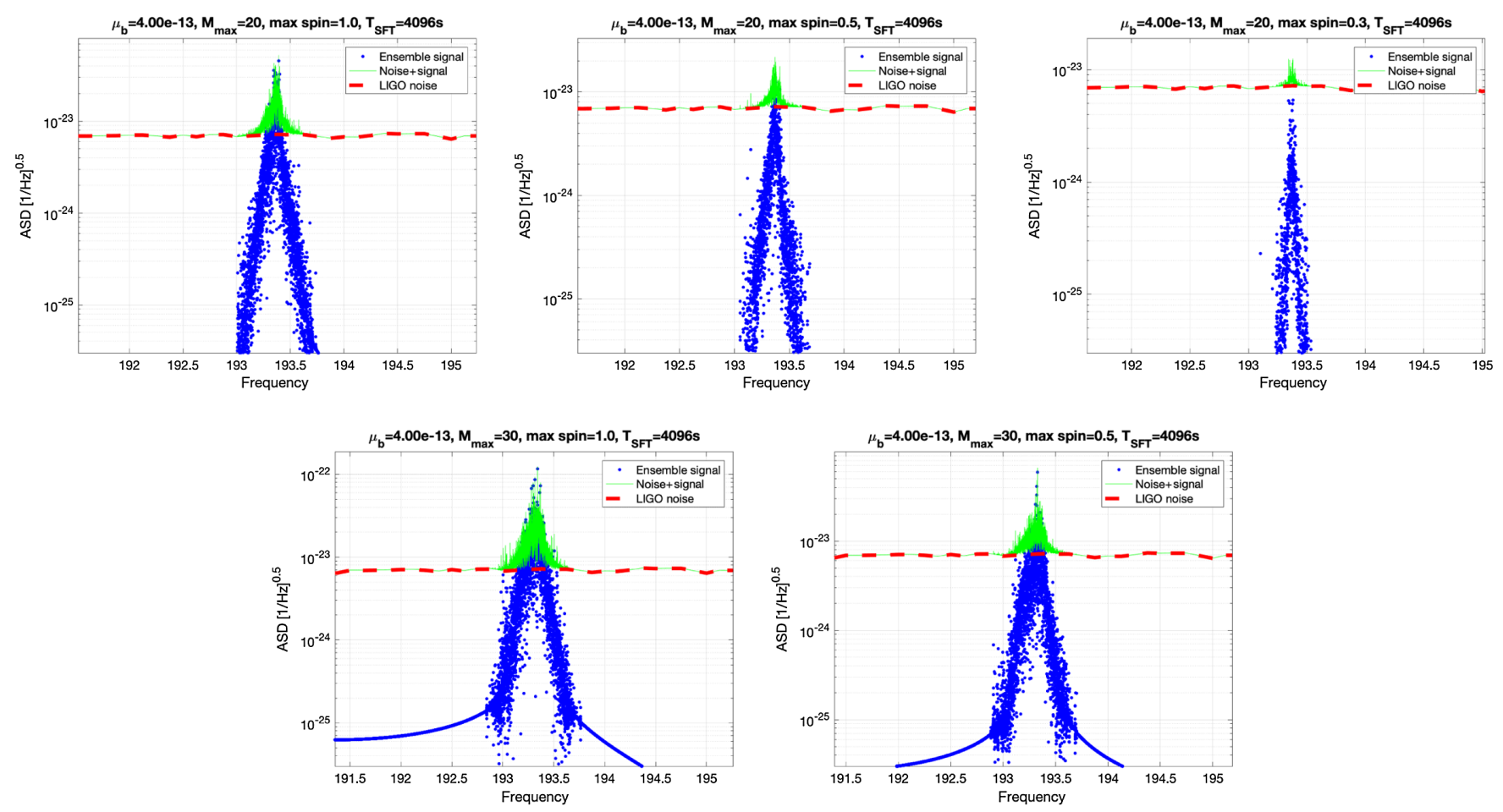

FIG. 44. The amplitude spectral density of the LIGO O2 data alone (dashed red line) and of an ensemble signal (top green points) assuming that $\mu_{\mathrm{b}}=4 \times 10^{-13} \mathrm{eV}$ and a Galactic black hole population with maximum mass $20 \mathrm{M}_{\odot}$ (top plots) and maximum initial spins of $0.3,0.5$, and 1 , and maximum mass $30 \mathrm{M}_{\odot}$ (bottom plots) and maximum initial spins of 0.5 and 1 . The time baseline assumed is 4096 s, as used by the Freq Hough search in this frequency range [22]. 

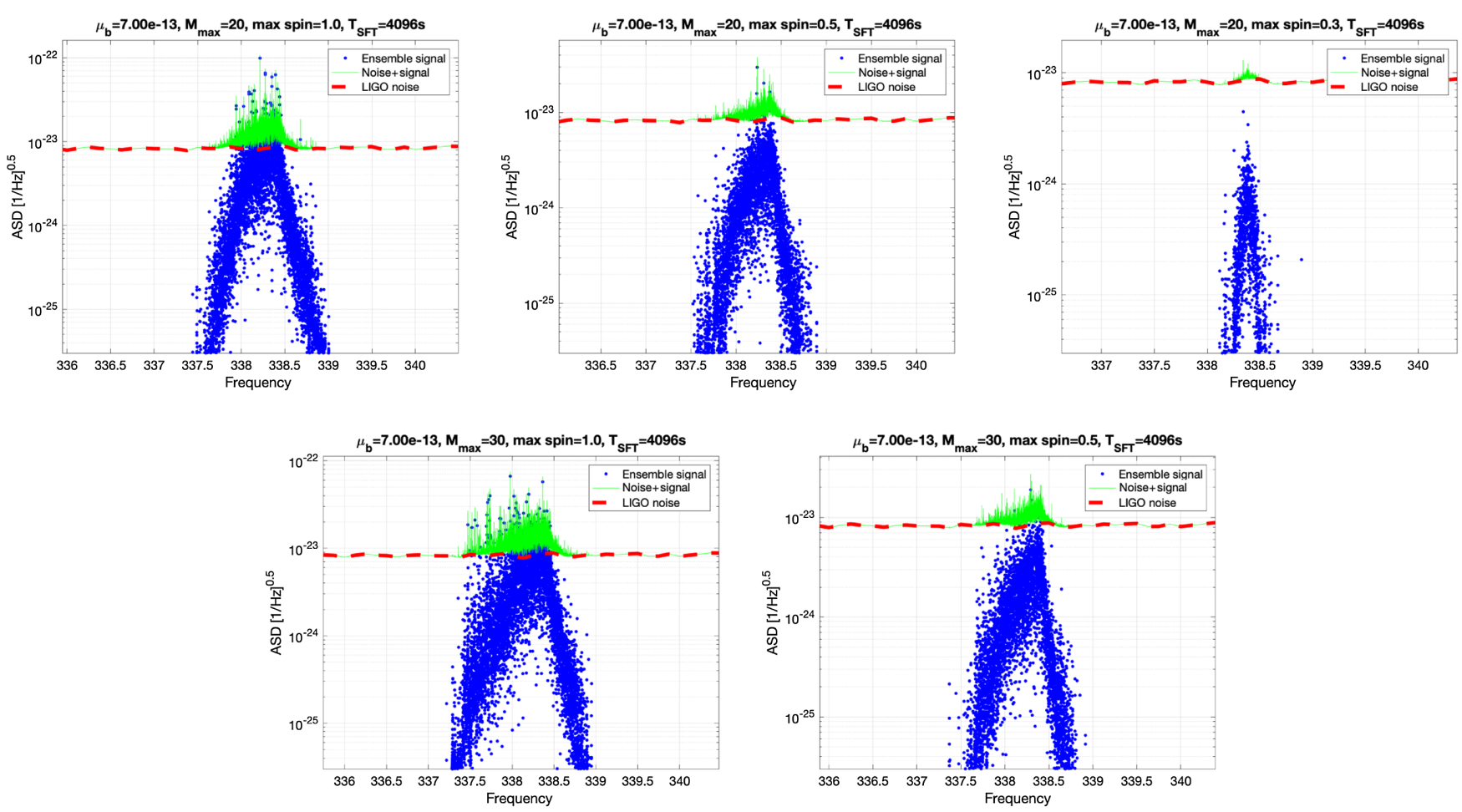

FIG. 45. The amplitude spectral density of the LIGO O2 data alone (dashed red line) and of an ensemble signal (top green points) assuming that $\mu_{\mathrm{b}}=7 \times 10^{-13} \mathrm{eV}$ and a Galactic black hole population with maximum mass $20 \mathrm{M}_{\odot}$ (top plots) and maximum initial spins of $0.3,0.5$, and 1 , and maximum mass $30 \mathrm{M}_{\odot}$ (bottom plots) and maximum initial spins of 0.5 and 1 . The time baseline assumed is 4096 s, as used by the Freq Hough search in this frequency range [22].
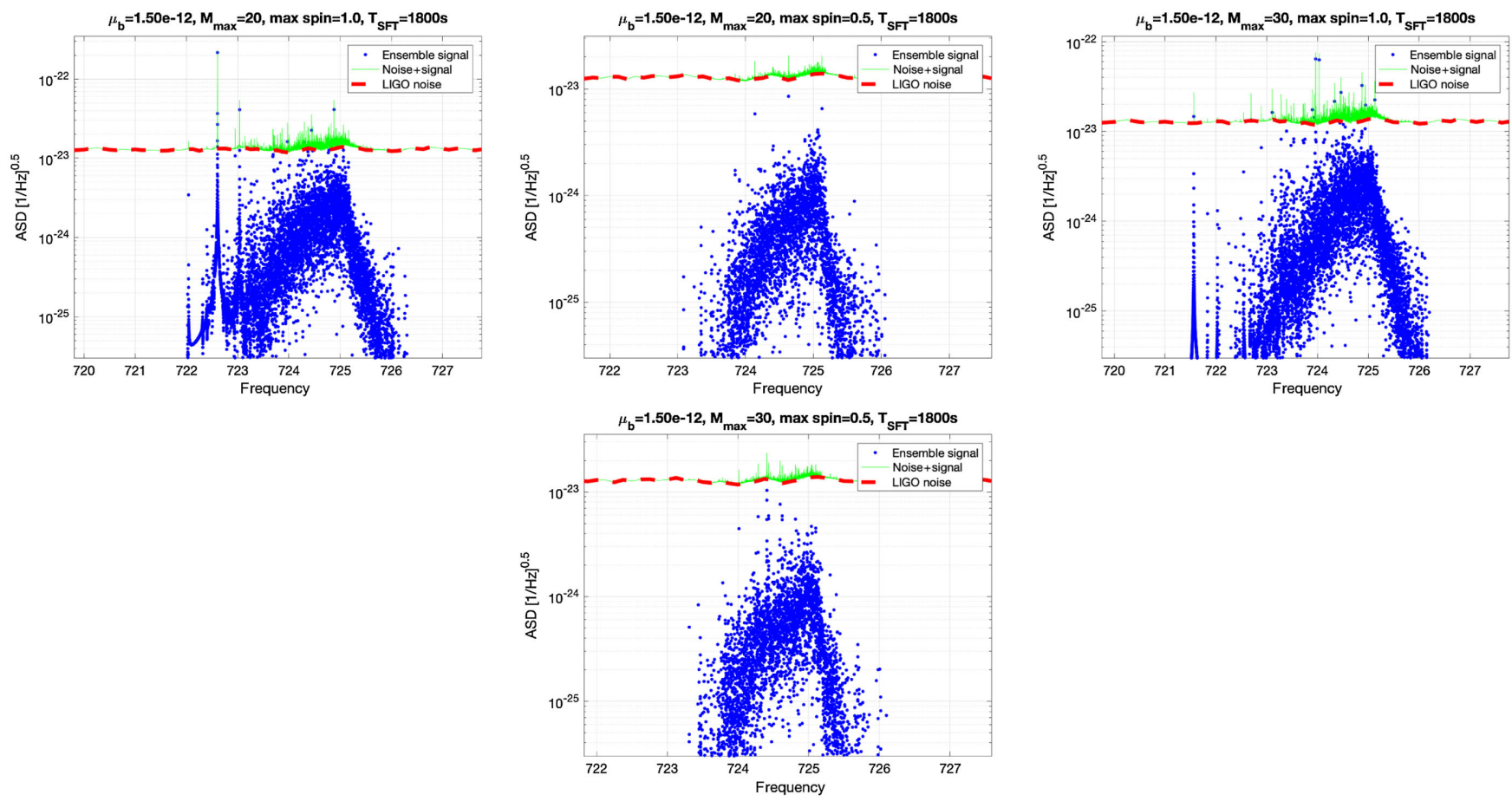

FIG. 46. The amplitude spectral density of the LIGO O2 data alone (dashed red line) and of an ensemble signal (top green points) assuming that $\mu_{\mathrm{b}}=1.5 \times 10^{-12} \mathrm{eV}$ and a Galactic black hole population with maximum mass $20 \mathrm{M}_{\odot}$ or $30 \mathrm{M}_{\odot}$ and maximum initial spins of 0.5 and 1 . The time baseline assumed is $1800 \mathrm{~s}$, close to what is used by the Freq Hough search in this frequency range [22]. The distinct excess at low frequencies arises from an exceptionally young and nearby black hole. 

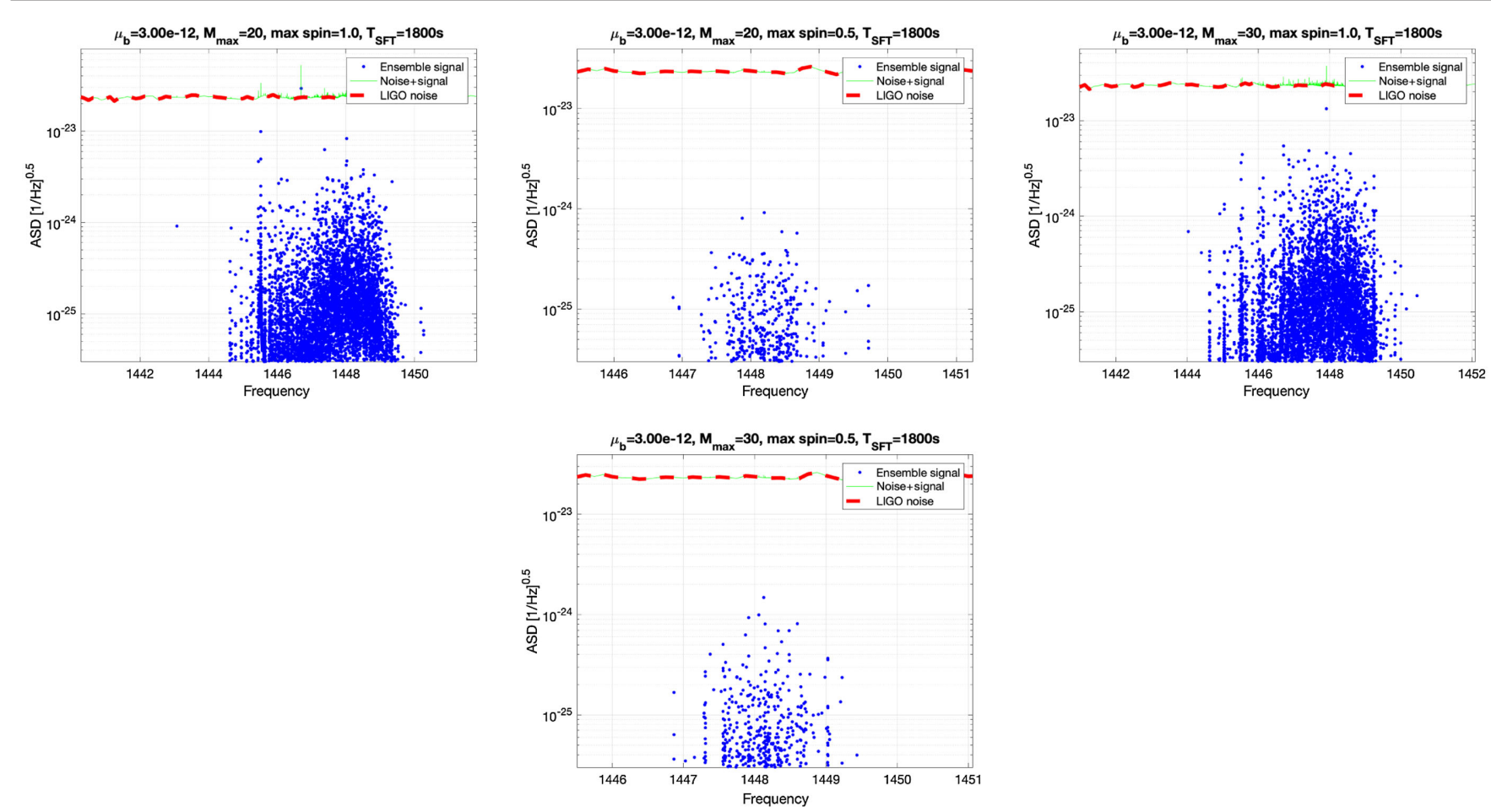

FIG. 47. The amplitude spectral density of the LIGO O2 data alone (dashed red line) and of an ensemble signal (top green points) assuming that $\mu_{\mathrm{b}}=3 \times 10^{-12} \mathrm{eV}$ and a Galactic black hole population with maximum mass $20 \mathrm{M}_{\odot}$ or $30 \mathrm{M}_{\odot}$ and maximum initial spins of 0.5 and 1. The time baseline assumed is $1800 \mathrm{~s}$, close to what is used by the Freq Hough search in this frequency range [22].

mass and maximum initial black hole spin. The time baseline $T_{S F T}$ at the range of frequencies corresponding to the range of boson masses is chosen to reflect existing search strategies. The values of the boson mass, maximum black hole mass, and maximum black hole spin, as well as $T_{S F T}$, are indicated in the figure titles.
[1] B. P. Abbott et al., Phys. Rev. X 9, 031040 (2019).

[2] K. Riles, Mod. Phys. Lett. A 32, 1730035 (2017).

[3] A. Arvanitaki, S. Dimopoulos, S. Dubovsky, N. Kaloper, and J. March-Russell, Phys. Rev. D 81, 123530 (2010).

[4] A. Arvanitaki and S. Dubovsky, Phys. Rev. D 83, 044026 (2011).

[5] A. Pierce, K. Riles, and Y. Zhao, Phys. Rev. Lett. 121, 061102 (2018).

[6] H.-K. Guo, K. Riles, F.-W. Yang, and Y. Zhao, Commun. Phys. 2, 155 (2019).

[7] C. J. Horowitz, M. A. Papa, and S. Reddy, Phys. Lett. B 800, 135072 (2020).

[8] R. D. Peccei and H. R. Quinn, Phys. Rev. Lett. 38, 1440 (1977).

[9] S. Weinberg, Phys. Rev. Lett. 40, 223 (1978).

[10] F. Wilczek, Phys. Rev. Lett. 40, 279 (1978).

[11] R. Essig et al., arXiv:1311.0029.

[12] A. Arvanitaki, M. Baryakhtar, and X. Huang, Phys. Rev. D 91, 084011 (2015).

[13] A. Arvanitaki, M. Baryakhtar, S. Dimopoulos, S. Dubovsky, and R. Lasenby, Phys. Rev. D 95, 043001 (2017).
[14] R. Brito, S. Ghosh, E. Barausse, E. Berti, V. Cardoso, I. Dvorkin, A. Klein, and P. Pani, Phys. Rev. D 96, 064050 (2017).

[15] R. Brito, S. Ghosh, E. Barausse, E. Berti, V. Cardoso, I. Dvorkin, A. Klein, and P. Pani, Phys. Rev. Lett. 119, 131101 (2017).

[16] M. C. Miller and J. M. Miller, Phys. Rep. 548, 1 (2015).

[17] J. E. McClintock, R. Narayan, and J. F. Steiner, Space Sci. Rev. 183, 295 (2014).

[18] V. Cardoso, O. J. C. Dias, G. S. Hartnett, M. Middleton, P. Pani, and J. E. Santos, J. Cosmol. Astropart. Phys. 03 (2018) 043.

[19] L. Tsukada, T. Callister, A. Matas, and P. Meyers, Phys. Rev. D 99, 103015 (2019).

[20] V. Dergachev and M. A. Papa, Phys. Rev. Lett. 123, 101101 (2019).

[21] V. Dergachev and M. A. Papa, Phys. Rev. D 101, 022001 (2020).

[22] C. Palomba et al., Phys. Rev. Lett. 123, 171101 (2019). 
[23] B. P. Abbott et al. (LIGO Scientific and Virgo Collaborations), Phys. Rev. D 96, 122004 (2017).

[24] J. G. Rosa and S. R. Dolan, Phys. Rev. D 85, 044043 (2012).

[25] P. Pani, V. Cardoso, L. Gualtieri, E. Berti, and A. Ishibashi, Phys. Rev. D 86, 104017 (2012).

[26] S. Endlich and R. Penco, J. High Energy Phys. 05 (2017) 052.

[27] M. Baryakhtar, R. Lasenby, and M. Teo, Phys. Rev. D 96, 035019 (2017).

[28] W. E. East, Phys. Rev. D 96, 024004 (2017).

[29] R. Brito, S. Grillo, and P. Pani, Phys. Rev. Lett. 124, 211101 (2020).

[30] R. Brito, V. Cardoso, and P. Pani, Lect. Notes Phys. 971, 1 (2015).

[31] Y. B. Zeldovich, JETP Lett. 14, 180 (1971).

[32] C. W. Misner, Phys. Rev. Lett. 28, 994 (1972).

[33] A. A. Starobinskii, Sov. Phys. JETP 37, 28 (1973).

[34] T. Damour, N. Deruelle, and R. Ruffini, Lett. Nuovo Cimento 15, 257 (1976).

[35] I. M. Ternov, V. R. Khalilov, G. A. Chizhov, and A. B. Gaina, Izv. Vyssh. Uchebn. Zaved., Fiz. 21N9, 109 (1978) [Sov. Phys. J. 21, 1200 (1978)].

[36] T. J. M. Zouros and D. M. Eardley, Ann. Phys. (N.Y.) 118, 139 (1979).

[37] S. L. Detweiler, Phys. Rev. D 22, 2323 (1980).

[38] A. Gruzinov, arXiv:1604.06422.

[39] M. Baryakhtar, M. Galanis, R. Lasenby, and O. Simon (to be published).

[40] H. Yoshino and H. Kodama, Prog. Theor. Exp. Phys. 128, 153 (2012).

[41] H. Yoshino and H. Kodama, Classical Quantum Gravity 32, 214001 (2015).

[42] S. Dolan, Phys. Rev. D 76, 084001 (2007).

[43] D. Baumann, H. S. Chia, and R. A. Porto, Phys. Rev. D 99, 044001 (2019).

[44] R. Brito, V. Cardoso, and P. Pani, Classical Quantum Gravity 32, 134001 (2015).

[45] H. Yoshino and H. Kodama, Prog. Theor. Exp. Phys. 2014, 043E02 (2014).

[46] B. P. Abbott (LIGO Scientific and Virgo Collaborations), Phys. Rev. D 96, 122004 (2017).

[47] B. P. Abbott et al., Phys. Rev. D 96, 062002 (2017).

[48] P. Bosch, S. R. Green, and L. Lehner, Phys. Rev. Lett. 116, 141102 (2016).

[49] S. L. Shapiro and S. A. Teukolsky, Black Holes, White Dwarfs, and Neutron Stars: The Physics of Compact Objects (Wiley, New York, 1983).

[50] D. P. Caputo, N. de Vries, A. Patruno, and S. P. Zwart, Mon. Not. R. Astron. Soc. 468, 4000 (2017).

[51] A. Heger, C. L. Fryer, S. E. Woosley, N. Langer, and D. H. Hartmann, Astrophys. J. 591, 288 (2003).

[52] W.-Q. Zhang, S. E. Woosley, and A. Heger, Astrophys. J. 679, 639 (2008).

[53] N. Prantzos and S. Boissier, Astron. Astrophys. 406, 259 (2003).

[54] R. Diehl et al., Nature (London) 439, 45 (2006).

[55] A. Olejak, K. Belczynski, T. Bulik, and M. Sobolewska, Astron. Astrophys. 638, A94 (2020).

[56] E. Agol and M. Kamionkowski, Mon. Not. R. Astron. Soc. 334, 553 (2002).
[57] G. Wiktorowicz, Ł. Wyrzykowski, M. Chruslinska, J. Klencki, K. A. Rybicki, and K. Belczynski, Astrophys. J. 885, 1 (2019).

[58] D. Tsuna, N. Kawanaka, and T. Totani, Mon. Not. R. Astron. Soc. 477, 791 (2018).

[59] D. Tsuna and N. Kawanaka, Mon. Not. R. Astron. Soc. 488, 2099 (2019).

[60] T. C. Licquia, and J. A. Newman, Astrophys. J. 806, 96 (2015).

[61] Y. Sofue, Publ. Astron. Soc. Jpn. 65, 118 (2013).

[62] D. M. Nataf, Pub. Astron. Soc. Aust. 33, e023 (2016).

[63] A. Irrgang, Astron. Astrophys. 549, A137 (2013).

[64] A. Kunder et al., Astron. J. 143, 57 (2012).

[65] C. Fryer, A. Burrows, and W. Benz, Astrophys. J. 496, 333 (1998).

[66] J. Nordhaus, T. D. Brandt, A. Burrows, E. Livne, and C. D. Ott, Phys. Rev. D 82, 103016 (2010).

[67] H. T. Janka, Mon. Not. R. Astron. Soc. 434, 1355 (2013).

[68] I. Mandel, Mon. Not. R. Astron. Soc. 456, 578 (2016).

[69] P. Atri et al., Mon. Not. R. Astron. Soc. 489, 3116 (2019).

[70] S. Repetto, M. B. Davies, and S. Sigurdsson, Mon. Not. R. Astron. Soc. 425, 2799 (2012).

[71] E. E. Salpeter, Astrophys. J. 121, 161 (1955).

[72] N. Bastian, K. R. Covey, and M. R. Meyer, Annu. Rev, Astron. Astrophys. 48, 339 (2010).

[73] C. Wegg, O. Gerhard, and M. Portail, Astrophys. J. Lett. 843, L5 (2017).

[74] C. S. Reynolds, Space Sci. Rev. 183, 277 (2014).

[75] J. Liu et al., Nature (London) 575, 618 (2019).

[76] K. El-Badry and E. Quataert, Mon. Not. R. Astron. Soc. Lett. 493, L22 (2020).

[77] J. Liu et al., arXiv:2005.12595.

[78] M. Fishbach and D. E. Holz, Astrophys. J. 851, L25 (2017).

[79] B. P. Abbott et al., Astrophys. J. Lett. 882, L24 (2019).

[80] J. Roulet and M. Zaldarriaga, Mon. Not. R. Astron. Soc. 484, 4216 (2019).

[81] B. P. Abbott et al., Astrophys. J. Lett. 818, L22 (2016).

[82] C. Belczynski, D. E. Holz, T. Bulik, and R. O'Shaughnessy, Nature (London) 534, 512 (2016).

[83] D. Toyouchi and M. Chiba, Astrophys. J. 855, 104 (2018).

[84] M. Ness and K. Freeman, Pub. Astron. Soc. Aust. 33, e022 (2016).

[85] J. Mackereth et al., Mon. Not. R. Astron. Soc. 471, 3057 (2017).

[86] J. Fuller and L. Ma, Astrophys. J. Lett. 881, L1 (2019).

[87] C.-A. Faucher-Giguere and V. M. Kaspi, Astrophys. J. 643, 332 (2006).

[88] S.-C. Yoon and N. Langer, Astron. Astrophys. 443, 643 (2005).

[89] S. Woosley and A. Heger, Astrophys. J. 637, 914 (2006).

[90] D. Wysocki, J. Lange, and R. O’Shaughnessy, Phys. Rev. D 100, 043012 (2019).

[91] E. M. Méndez and M. Cantiello, New Astron. 44, 58 (2016).

[92] I. Ribas, C. Jordi, F. Vilardell, E. L. Fitzpatrick, R. W. Hilditch, and E. F. Guinan, Astrophys. J. Lett. 635, L37 (2005).

[93] A. W. McConnachie, Astron. J. 144, 4 (2012).

[94] G. Pietrzyński et al., Nature (London) 567, 200 (2019). 
[95] D. Graczyk et al., Astrophys. J. 780, 59 (2014).

[96] See https://www.aei.mpg.de/continuouswaves/ arxiv200303359.

[97] B. P. Abbott et al., Phys. Rev. D 100, 024004 (2019).

[98] F. Ozel, D. Psaltis, R. Narayan, and J. E. McClintock, Astrophys. J. 725, 1918 (2010).

[99] B. Allen and J. D. Romano, Phys. Rev. D 59, 102001 (1999).

[100] S. Walsh et al., Phys. Rev. D 94, 124010 (2016).

[101] S. Walsh, K. Wette, M. A. Papa, and R. Prix, Phys. Rev. D 99, 082004 (2019).

[102] O. J. Piccinni, P. Astone, S. D’Antonio, S. Frasca, G. Intini, P. Leaci, S. Mastrogiovanni, A. Miller, C. Palomba, and A. Singhal, Classical Quantum Gravity 36, 015008 (2019).

[103] V. Dergachev, M. A. Papa, B. Steltner, and H.-B. Eggenstein, Phys. Rev. D 99, 084048 (2019).

[104] J. Ming et al., Phys. Rev. D 100, 024063 (2019).

[105] P. Astone, S. Frasca, and C. Palomba, Classical Quantum Gravity 22, S1197 (2005).
[106] P. Astone (private communication).

[107] M. Isi, L. Sun, R. Brito, and A. Melatos, Phys. Rev. D 99, 084042 (2019).

[108] L. Sun, R. Brito, and M. Isi, Phys. Rev. D 101, 063020 (2020).

[109] L. Gou, J. E. McClintock, M. J. Reid, J. A. Orosz, J. F. Steiner, R. Narayan, J. Xiang, R. A. Remillard, K. A. Arnaud, and S. W. Davis, Astrophys. J. 742, 85 (2011).

[110] L. Gou, J. E. McClintock, R. A. Remillard, J. F. Steiner, M. J. Reid, J. A. Orosz, R. Narayan, M. Hanke, and J. García, Astrophys. J. 790, 29 (2014).

[111] N. Siemonsen and W. E. East, Phys. Rev. D 101, 024019 (2020).

[112] E. Berti, V. Cardoso, and M. Casals, Phys. Rev. D 73, 024013 (2006); 73, 109902(E) (2006).

[113] W. M. Farr, N. Sravan, A. Cantrell, L. Kreidberg, C. D. Bailyn, I. Mandel, and V. Kalogera, Astrophys. J. 741, 103 (2011).

[114] V. Dergachev and M. A. Papa, arXiv:2004.08334. 